\title{
Dearomative Indole Cycloaddition Reactions of Aza-Oxyallyl Cationic Intermediates: Modular Access to Pyrroloindolines
}

\author{
Arjun Acharya, Devendar Anumandla, and Christopher S. Jeffrey* \\ Department of Chemistry, University of Nevada, Reno, Nevada, 89557-0216, United States
}

\section{Table of Contents}

General Experimental

Experimental procedures for the preparation of indoles

General experimental procedures and tabulated characterization data

for $(3+2)$-cycloadditions

Experimental procedures and tabulated data for the formal

synthesis of Physostigmine and Debromoflustramine B

References

Copies of $1 \mathrm{H}$ and 13C NMR spectra
S7-S23

S23-S27

S28

S1

S2-S6

S29-S99 


\section{General Experimental:}

All reactions were carried out under an atmosphere of nitrogen in oven-dried glassware with magnetic stirring, unless otherwise specified. Hexafluoro isopropanol (HFIP) and 2,2,3,3,tetrafluoropropanol (TFP) were purchased from SynQuest. All other reagents and solvents were purchased from Sigma-Aldrich Chemical Company and used without any further purification. TLC information was recorded on Silicycle glass 60 F254 plates and developed by staining with $\mathrm{KMnO}_{4}$ or ceric ammonium molybdate (CAM). Purification of reaction products was carried out by flash chromatography using Silicycle Siliaflash ${ }^{\circledR}$ P60 (230-400 mesh). ${ }^{1} \mathrm{H}$ NMR and ${ }^{13} \mathrm{C}$ NMR spectra were measured on Varian MR400 $(400 \mathrm{MHz})$, or Varian $500(500 \mathrm{MHz})$ spectrometers and are reported in ppm ( $\mathrm{s}=$ singlet, $\mathrm{d}=$ doublet, $\mathrm{t}=$ triplet, $\mathrm{q}=$ quartet, $\mathrm{m}=$ multiplet, br=broad; integration; coupling constant(s) in Hz), using TMS as an internal standard (TMS at $0.00 \mathrm{ppm}$ ) in $\mathrm{CDCl}_{3} .{ }^{13} \mathrm{C}$ NMR spectra were recorded on V400 or V500 spectrometer and reported in ppm using solvent as an internal standard $\left(\mathrm{CDCl}_{3}\right.$ at $77.16 \mathrm{ppm}$ and $\mathrm{CD}_{3} \mathrm{OD}$ at 49.86 ppm). Infrared (IR) spectra were recorded on a Nicolet 6700 FT-IR with a diamond ATR and data are reported as $\mathrm{cm}^{-1}(\mathrm{br}=$ broad, $\mathrm{s}=$ strong). High-resolution mass spectra (HRMS) were obtained using an Agilent 6230 TOF LC/MS with an electrospray (ESI) source with purine and HP-0921 as an internal calibrants. 


\section{Synthesis of 1,3-disubstituted indoles:}

1,3-disubstituted indoles [Substrate, table 2, entry $11-10,{ }^{(1)} 1,3,9,12,13,{ }^{(2)} 15,{ }^{(3)} 14,{ }^{(4)} 7^{(5)}$ and $8^{(6)}$ ] were synthesized using known procedures from literature.<smiles>CC(C)c1cn(C)c2ccccc12</smiles>

Substrate, table 2, entry 1<smiles>Cn1cc(CC[SeH2])c2ccccc21</smiles>

Substrate, table 2, entry 5<smiles>Cc1cn(C)c2ccc(Br)cc12</smiles>

Substrate, table 2, entry 9<smiles>CCn1cc(C)c2ccccc21</smiles>

Substrate, table 2, Substrate, table 2, entry 13<smiles>COc1ccc2c(c1)c(C(C)C)cn2C</smiles>

Substrate, table 2, entry 2

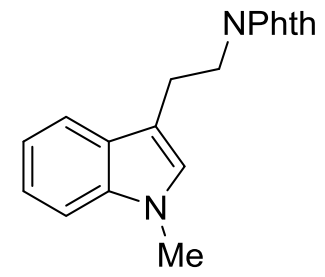

Substrate, table 2, entry 6<smiles>Cc1cn(Cc2ccccc2)c2ccc(Br)cc12</smiles>

Substrate, table 2, entry 10<smiles>C=CCn1cc(C)c2ccccc21</smiles>
entry 14<smiles>Cn1cc(C2CCCCC2)c2ccccc21</smiles>

Substrate, table 2, entry 3<smiles>COC(=O)Cc1cn(C)c2ccccc12</smiles>

Substrate, table 2, entry 7<smiles>Cc1cn(Cc2ccccc2)c2ccccc12</smiles>

Substrate, table 2, entry 11<smiles>Cc1cn([13CH3])c2ccccc12</smiles>

Substrate, table 2, entry 15<smiles>Cn1cc(Cc2ccccc2)c2ccccc21</smiles>

Substrate, table 2 , entry 4<smiles>Cn1cc(CC#N)c2ccccc21</smiles>

Substrate, table 2, entry 8<smiles>c1ccc(Cn2cc(C3CCCCC3)c3ccccc32)cc1</smiles>

Substrate, table 2, entry 12

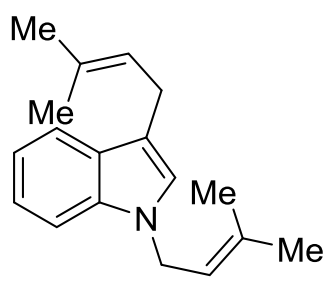

17 


\section{5-Ethoxy-1-methyl-3-(propan-2-yl)-1H-indole (Substrate, table 2, entry 2)}

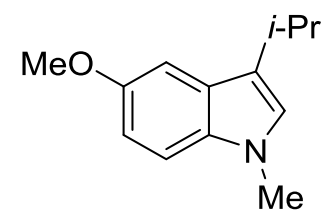

To a solution of triethylsilane $(1.6 \mathrm{ml}, 10.2 \mathrm{mmol})$, trifluoroacetic acid $(0.4 \mathrm{ml}, 5.1 \mathrm{mmol})$ in toluene $(10 \mathrm{~mL})$ was added a solution of 5-methoxy- $1 H$-indole $(0.5 \mathrm{~g}, 3.4 \mathrm{mmol})$ and acetone $(0.5 \mathrm{~mL}, 6.7 \mathrm{mmol})$ in toluene $(5 \mathrm{~mL})$ was added dropwise at room temperature. The resulting solution was stirred for overnight at $50{ }^{\circ} \mathrm{C}$ and the solution was quenched with saturated aqueous sodium bicarbonate $(10 \mathrm{~mL})$. The mixture was extracted with diethyl ether and the organic layer was separated, dried over $\mathrm{Na}_{2} \mathrm{SO}_{4}$, concentrated under redeced pressure. and purified using column chromatography to give 3-isopropyl-5-methoxy- $1 H$-indole in $79 \%$ yield $(0.35 \mathrm{~g}, 2.7$ mmol) as a pale yellow oil. $R_{f}=0.25$ (6:4, hexanes: ethyl acetate); ${ }^{1} \mathrm{H}$ NMR (500 $\left.\mathrm{MHz}, \mathrm{CDCl}_{3}\right)$ : $\delta 7.75(\mathrm{~s}, 1 \mathrm{H}), 7.23(\mathrm{dd}, J=8.8,0.5 \mathrm{~Hz}, 1 \mathrm{H}), 7.09(\mathrm{dd}, J=2.5,0.7 \mathrm{~Hz}, 1 \mathrm{H}), 6.95-6.90(\mathrm{~m}, 1 \mathrm{H})$, $6.85(\mathrm{dd}, J=8.8,2.5 \mathrm{~Hz}, 1 \mathrm{H}), 3.87$ (s, 3H), 3.16 (hept.d, $J=6.8,0.8 \mathrm{~Hz}, 1 \mathrm{H}), 1.35$ (d, $J=6.8$ $\mathrm{Hz}, 6 \mathrm{H}) ;{ }^{13} \mathrm{C}$ NMR $\left(126 \mathrm{MHz}, \mathrm{CDCl}_{3}\right): \delta 153.7,131.8,127.1,123.7,120.1,111.8,111.7,101.5$, 56.0, 25.4, 23.2; FT-IR (neat): 3411, 2957, 2868, 2830, 1680, 1656, 1622, 1582, 1514, 1482 , 1453, 1438, 1418, 1382, 1302, 1283, 1261, $1208 \mathrm{~cm}^{-1}$; HRESI-MS: calculated for $\mathrm{C}_{12} \mathrm{H}_{16} \mathrm{NO}$ $(\mathrm{M}+\mathrm{H})^{+}$190.1226, observed 190.1220.

To a solution of $\mathrm{NaH}(60 \%$ dispersion oil in mineral oil, $89 \mathrm{mg}, 2.22 \mathrm{mmol})$ in $\mathrm{DMF}$ at $0{ }^{\circ} \mathrm{C}$ was added 3-isopropyl-5-methoxy- $1 H$-indole $(0.35 \mathrm{~g}, 1.85 \mathrm{mmol})$ and the mixture was stirred for 30 min at room temperature. The reaction mixture was cooled to $0{ }^{\circ} \mathrm{C}$ and was added methyl iodide (138 $\mu \mathrm{L}, 2.22 \mathrm{mmol})$ dropwise. The mixture was stirred at room tempearture for $3 \mathrm{~h}$. The crude reaction mixture was treated with saturated ammonium chloride solution $(10 \mathrm{~mL})$ and the aqueous phase was extracted with diethyl ether $(3 \times 20)$. The combined organic phases were dried over anhydrous sodium sulfate and the solvent was removed under reduced pressure. The crude product was purified by column chromatography (5\% ethyl acetate in hexane) to provide title compound in $79 \%$ yield $(1.08 \mathrm{~g}, 4.3 \mathrm{mmol})$ as a pale yellow oil. $R_{f}=0.57(9: 1$, hexanes: ethyl acetate); ${ }^{1} \mathrm{H} \mathrm{NMR}\left(500 \mathrm{MHz}, \mathrm{CDCl}_{3}\right): \delta 7.16(\mathrm{dd}, J=8.9,0.6 \mathrm{~Hz}, 1 \mathrm{H}), 7.08(\mathrm{~d}, J=2.5 \mathrm{~Hz}$, 1H), 7.07 (dd, $J=2.4,0.6 \mathrm{~Hz}, 1 \mathrm{H}), 6.87$ (dd, $J=8.8,2.4 \mathrm{~Hz}, 1 \mathrm{H}), 6.77$ (br.s, $1 \mathrm{H}), 3.86$ (s, 3H), 
3.69 (s, 3H), 3.15 (hept.d, $J=6.8,0.8, \mathrm{~Hz}, 1 \mathrm{H}), 1.34$ (d, $J=7.0 \mathrm{~Hz}, 6 \mathrm{H}) ;{ }^{13} \mathrm{C} \mathrm{NMR}(126 \mathrm{MHz}$, $\left.\mathrm{CDCl}_{3}\right): \delta 153.5,132.8,127.4,124.9,122.0,111.5,110.0,101.7,56.1,32.7,25.5,23.5$; FT-IR (neat): 2954, 2967, 2830, 1620, 1575, 1488, 1465, 1451, 1422, 1377, 1362, 1328, 1309, 1280, 1257, 1238, $1219 \mathrm{~cm}^{-1}$; HRESI-MS: calculated for $\mathrm{C}_{13} \mathrm{H}_{18} \mathrm{NO}(\mathrm{M}+\mathrm{H})^{+}$204.1383, observed 204.1380 .

\section{3-Benzyl-1-methyl-1H-indole (Substrate, table 2, entry 4)}<smiles>Cn1cc(Br)c2ccccc21</smiles>

To a solution of triethylsilane $(2.4 \mathrm{ml}, 18.3 \mathrm{mmol})$, trifluoroacetic acid $(0.7 \mathrm{ml}, 9.15 \mathrm{mmol})$ in toluene $(5 \mathrm{~mL})$ was added a solution of $N$-methyl- $1 H$-indole $(0.8 \mathrm{~g}, 6.1 \mathrm{mmol})$ and benzaldehyde $(0.68 \mathrm{~mL}, 6.7 \mathrm{mmol})$ in toluene $(5 \mathrm{~mL})$ was added dropwise at room temperature. The resulting solution was stirred for overnight at $50{ }^{\circ} \mathrm{C}$ and the solution was quenched with saturated aqueous sodium bicarbonate $(10 \mathrm{~mL})$. The mixture was extracted with diethyl ether and the organic layer was separated, dried over $\mathrm{Na}_{2} \mathrm{SO}_{4}$, concentrated under redeced pressure. and purified using column chromatography to give 3-benzyl-1-methyl- $1 H$-indole in $67 \%$ yield $(0.9 \mathrm{~g}, 4.01 \mathrm{mmol})$ as a pale yellow oil. The spectral data were matched with the literature data. ${ }^{7}$

\section{3-[2-[[(1,1-Dimethylethyl)dimethylsilyl]oxy]ethyl]-1-methyl-1H-Indole (Substrate, table 2, entry 5)}

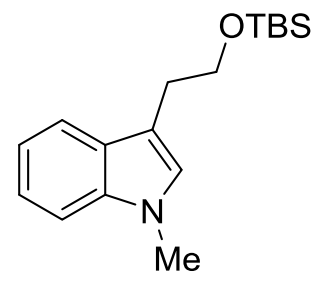

To a mixture of 2-(1-methyl-1H-indol-3-yl)ethan-1-ol (0.8 g, $4.56 \mathrm{mmol})$ and imidazole $(0.37 \mathrm{~g}$, $5.5 \mathrm{mmol})$ in THF $(10 \mathrm{~mL})$ was added $t$-butyldimethylsilyl chloride $(0.83 \mathrm{~g}, 5.5 \mathrm{mmol})$ and the reaction was stirred at room temperature for overnight. The mixture was diluted with $30 \mathrm{~mL}$ of EtOAc and then washed with water and brine. The organic layer was dried over anhydrous 
sodium sulfate. The solvent was removed under reduced pressure and was purified by column chromatography (5\% EtOAc in hexanes) to give title compound in $80 \%$ yield $(1.05 \mathrm{~g}, 3.65$ mmol) as a a pale yellow oil. $R_{f}=0.73\left(8: 2\right.$, hexanes: ethyl acetate); ${ }^{1} \mathrm{H}$ NMR (500 MHz, $\left.\mathrm{CDCl}_{3}\right): \delta 7.62(\mathrm{dq}, J=7.8,0.9 \mathrm{~Hz}, 1 \mathrm{H}), 7.30(\mathrm{dt}, J=8.2,1.0 \mathrm{~Hz}, 1 \mathrm{H}), 7.23(\mathrm{ddd}, J=8.2,7.0$, $1.1 \mathrm{~Hz}, 1 \mathrm{H}), 7.12$ (ddd, $J=8.0,6.9,1.1 \mathrm{~Hz}, 1 \mathrm{H}), 6.91(\mathrm{~s}, 1 \mathrm{H}), 3.88$ (t, $J=7.5 \mathrm{~Hz}, 2 \mathrm{H}), 3.76$ (s, $3 \mathrm{H}), 3.01(\mathrm{t}, J=7.5 \mathrm{~Hz}, 2 \mathrm{H}), 0.93(\mathrm{~s}, 9 \mathrm{H}), 0.07(\mathrm{~s}, 6 \mathrm{H}) ;{ }^{13} \mathrm{C} \mathrm{NMR}(126 \mathrm{MHz}, \mathrm{CDCl} 3): \delta 136.9$, 128.2, 126.9, 121.5, 119.0, 118.7, 111.6, 109.2, 64.2, 32.6, 29.0, 26.1, 18.5, -5.2; FT-IR (neat): 3055, 2952, 2927, 2855, 1616, 1554, 1471, 1424, 1373, 1360, 1327, 1249, $1216 \mathrm{~cm}^{-1}$; HRESIMS: calculated for $\mathrm{C}_{17} \mathrm{H}_{27} \mathrm{NOSiNa}(\mathrm{M}+\mathrm{Na})^{+} 312.1754$, observed 312.1766 .

\section{2-[2-(1-Methyl-1H-indol-3-yl)ethyl]-1H-Isoindole-1,3(2H)-dione (Substrate, table 2, entry 6)}<smiles>Cn1cc(CCN2C(=O)c3ccccc3C2=O)c2ccccc21</smiles>

To a stirring solution of 1-methyl-3-(2-bromoethyl)- $1 H$-indole ${ }^{8}(0.5 \mathrm{~g}, 2.01 \mathrm{mmol})$ in DMF (10 $\mathrm{mL})$ was added potassium phthalimide $(0.45 \mathrm{mg}, 2.41 \mathrm{mmol})$ in portions at room temperature, and refluxed for $10 \mathrm{~h}$. The reaction mixture was poured into water $(50 \mathrm{~mL})$ and extracted with dichloromethane $(2 \times 50 \mathrm{~mL})$. The combined organic layer was washed with water, brine, and dried over anhydrous $\mathrm{Na}_{2} \mathrm{SO}_{4}$, filtered and concentrated in reduced pressure and purified by column chromatography to give title compound in $74 \%$ yield as pale yellow solid. The spectral data were matched with the literature data. ${ }^{9}$ 


\section{1,3-Bis(3-methylbut-2-en-1-yl)-1H-indole (17)}

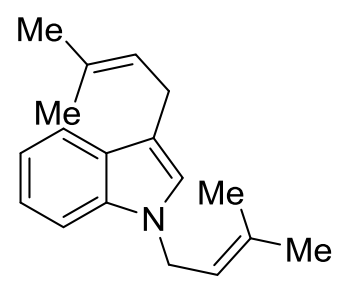

3-(3-methylbut-2-en-1-yl)-1H-indole was prepared in 50\% yield according to the literature procedure. ${ }^{10}$ To a solution of $\mathrm{NaH}$ (60\% dispersion oil in mineral oil, $\left.0.43 \mathrm{~g}, 6.5 \mathrm{mmol}\right)$ in DMF at $0{ }^{\circ} \mathrm{C}$ was added 3-(3-methylbut-2-en-1-yl)- $1 H$-indole $(1 \mathrm{~g}, 5.4 \mathrm{mmol})$ and the mixture was stirred for $30 \mathrm{~min}$ at room temperature. The reaction mixture was cooled to $0{ }^{\circ} \mathrm{C}$ and was added 3,3-dimethylallyl bromide $(0.65 \mathrm{~mL}, 6.5 \mathrm{mmol})$ dropwise. The mixture was stirred at room tempearture for $3 \mathrm{~h}$. The crude reaction mixture was treated with saturated ammonium chloride solution $(20 \mathrm{~mL})$ and the aqueous phase was extracted with diethyl ether $(3 \times 50)$. The combined organic phase were dried over anhydrous sodium sulfate and the solvent was removed under reduced pressure. The crude product was purified by column chormatography $(5 \%$ ethylacetate in hexane) to provide title compound in $79 \%$ yield $(1.08 \mathrm{~g}, 4.3 \mathrm{mmol})$ as a pale yellow oil. $R_{f}=$ 0.58 (19:5, hexanes: ethyl acetate); ${ }^{1} \mathrm{H}$ NMR (500 MHz, $\left.\mathrm{CDCl}_{3}\right): \delta 7.57(\mathrm{dd}, J=7.9,1.7 \mathrm{~Hz}$, 1H), 7.27 (d, $J=8.2 \mathrm{~Hz}, 1 \mathrm{H}), 7.18$ (t, $J=7.5 \mathrm{~Hz}, 1 \mathrm{H}), 7.07$ (t, $J=7.9 \mathrm{~Hz}, 1 \mathrm{H}), 6.84(\mathrm{~s}, 1 \mathrm{H})$, $5.48-5.39(\mathrm{~m}, 1 \mathrm{H}), 5.40-5.32(\mathrm{~m}, 1 \mathrm{H}), 4.63(\mathrm{~d}, J=6.7 \mathrm{~Hz}, 2 \mathrm{H}), 3.44(\mathrm{~d}, J=7.1 \mathrm{~Hz}, 2 \mathrm{H}), 1.81$ (s, 3H), 1.77 (s, 3H), 1.75 (s, 6H); 13C NMR (126 MHz, CDCl3): $\delta$ 136.4, 135.6, 131.6, 128.0, 124.6, 123.4, 121.3, 120.4, 119.2, 118.5, 114.6, 109.4, 44.0, 25.8, 25.7, 24.1, 18.0, 17.8; FT-IR (neat): 3048, 2967, 2913, 1674, 1612, 1550, 1481, 1465, 1375, 1357, 1331, 1312, 1253, 1216 $\mathrm{cm}^{-1}$; HRESI-MS: calculated for $\mathrm{C}_{18} \mathrm{H}_{24} \mathrm{~N}(\mathrm{M}+\mathrm{H})^{+}$254.1903, observed 254.1904.

\section{Synthesis of $\alpha$-haloamides:}

$\alpha$-Haloamides $1, \mathbf{1 1}, \mathbf{1 3}, \mathbf{1 5}$ were prepared according to the literature procedure. ${ }^{11}$<smiles>CC(C)(Br)C(=O)NOCc1ccccc1</smiles><smiles>CCC(Br)C(=O)NOCc1ccccc1</smiles><smiles>O=C(NOCc1ccccc1)C1(Br)CCCCC1</smiles><smiles>O=C(NOCc1ccccc1)C(Cl)Cl</smiles> 
General Procedure A for the (3+2)-cycloaddition of indoles with halo amides: To a solution of indole 4 in HFIP (1.0 M) was added $\alpha$-haloamide 1 (1 equiv.) (1.2 equiv. for table 2 entry 4, 6-11, 16 and table 3 entry 4) and sodium carbonate (2 equiv.) and stirred at room temperature (table 3 , entry 1 and 3 at $50^{\circ} \mathrm{C}$ ) and the reaction progress monitored by TLC (8:2 or 2:1 hexanes:ethyl acetate) until complete consumption of the indole (reaction time: see substrate tables). The mixture was filtered through the short pad of celite and the filtrate was concentrated under reduced pressure. The residue was purified via flash column chromatography $(8: 2$ to $7: 3$, hexanes: ethyl acetate) to provide the desired cycloadducts.

( \pm )-(3aR,6aR)-6-(Phenylmethoxy)-2,4,4-trimethyl-3a,4,6,6a-tetrahydro-5H-furo[2,3b]pyrrol-5-one (3)

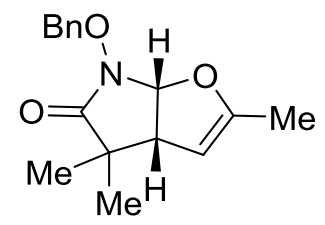

To a solution of the $\alpha$-haloamide $(100 \mathrm{mg}, 0.367 \mathrm{mmol})$ in $\mathrm{CF}_{3} \mathrm{CH}_{2} \mathrm{OH}$ and furan $[1: 1(\mathrm{v} / \mathrm{v}) 0.25$ $\mathrm{M}$ ] added triethylamine $(103 \mu \mathrm{L}, 0.72 \mathrm{mmol})$ dropwise at $0{ }^{\circ} \mathrm{C}$. The solution was then allowed to warm to room temperature and the reaction progress monitored by TLC (3:1 or 2:1 hexanes:ethyl acetate) until complete consumption of the haloamide. The volatiles were removed under reduced pressure and the crude residue purified by flash column chromatography (4:1 to $3: 1$ hexanes:ethyl acetate) to afford the pure cycloadducts in $64 \%$ yield as colorless oils. $R_{f}=0.55$ (6:4, hexanes: ethyl acetate); ${ }^{1} \mathrm{H} \mathrm{NMR}\left(500 \mathrm{MHz}, \mathrm{CDCl}_{3}\right): \delta 7.46-7.39(\mathrm{~m}, 2 \mathrm{H}), 7.40-7.32(\mathrm{~m}$, 3H), $5.06(\mathrm{~d}, J=10.9 \mathrm{~Hz}, 1 \mathrm{H}), 4.96$ (d, $J=10.8 \mathrm{~Hz}, 1 \mathrm{H}), 4.73(\mathrm{dq}, J=2.3,1.2 \mathrm{~Hz}, 1 \mathrm{H}), 4.42$ (d, $J=7.4 \mathrm{~Hz}, 1 \mathrm{H}), 4.37$ (ddq, $J=7.4,2.2,1.1 \mathrm{~Hz}, 1 \mathrm{H}), 1.76$ (t, $J=1.2 \mathrm{~Hz}, 3 \mathrm{H}), 1.23$ (s, 3H), 1.17 $(\mathrm{s}, 3 \mathrm{H}) ;{ }^{13} \mathrm{C} \mathrm{NMR}\left(101 \mathrm{MHz}, \mathrm{CDCl}_{3}\right): \delta 172.8,160.8,135.4,129.6,128.9,128.5,95.0,85.2$, 77.2, 63.4, 42.1, 25.7, 18.9, 13.6; FT-IR (neat): 3032, 2929, 2871, 1700, 1667, 1489, 1455, 1385, 1363, 1352, 1329, 1314, 1292, 1246, $1212 \mathrm{~cm}^{-1}$; HRESI-MS: calculated for $\mathrm{C}_{16} \mathrm{H}_{19} \mathrm{NO}_{3} \mathrm{Na}$ $(\mathrm{M}+\mathrm{Na})^{+}$296.1257, observed 296.1257. 
$( \pm)-(3 a R, 8 a S)-1-P h e n y l m e t h o x y-3,3,3 a, 8-t e t r a m e t h y l-3,3 a, 8,8 a-t e t r a h y d r o p y r r o l o[2,3-$ b]indol-2(1H)-one (5)

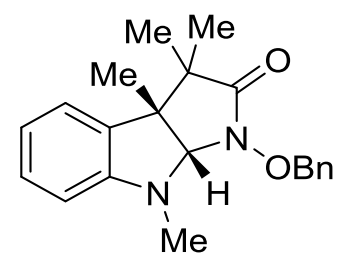

Prepared in $83 \%$ yield $(96.5 \mathrm{mg}, 0.29 \mathrm{mmol})$ as a colorless oil from the reaction of 1,3dimethylindole 4 (50 mg, $0.34 \mathrm{mmol}$ ) with 2-bromo-2-methyl- $N$-(phenylmethoxy)propanamide (93.4 mg, $0.34 \mathrm{mmol}$ ) via general procedure A. $R_{f}=0.3$ (8:2, hexanes: ethyl acetate); ${ }^{1} \mathrm{H}$ NMR $\left(500 \mathrm{MHz}, \mathrm{CDCl}_{3}\right): \delta 7.53-7.44(\mathrm{~m}, 2 \mathrm{H}), 7.44-7.33(\mathrm{~m}, 3 \mathrm{H}), 7.13(\mathrm{td}, J=7.7,1.3 \mathrm{~Hz}, 1 \mathrm{H})$, 6.95 (ddd, $J=7.4,1.3,0.5 \mathrm{~Hz}, 1 \mathrm{H}), 6.71$ (td, $J=7.4,1.0 \mathrm{~Hz}, 1 \mathrm{H}), 6.43$ (br.d, $J=7.9 \mathrm{~Hz}, 1 \mathrm{H}$ ), $5.17(\mathrm{~d}, J=10.6 \mathrm{~Hz}, 1 \mathrm{H}), 5.01(\mathrm{~d}, J=10.6 \mathrm{~Hz}, 1 \mathrm{H}), 4.32(\mathrm{~s}, 1 \mathrm{H}), 2.94(\mathrm{~s}, 3 \mathrm{H}), 1.21(\mathrm{~s}, 3 \mathrm{H}), 1.21$ $(\mathrm{s}, 3 \mathrm{H}), 1.00(\mathrm{~s}, 3 \mathrm{H}) ;{ }^{13} \mathrm{C} \mathrm{NMR}\left(126 \mathrm{MHz}, \mathrm{CDCl}_{3}\right): \delta 175.0,150.5,134.9,132.0,129.8,129.1$, 128.6, 128.6, 124.0, 118.4, 107.3, 88.4, 77.2, 51.5, 43.8, 34.7, 24.3, 22.0, 21.6; FT-IR (neat): $3031,2972,2931,2871,1702,1605,1487,1454,1428,1397,1373,1322,1307,1280,1211 \mathrm{~cm}^{-}$ 1. HRESI-MS: calculated for $\mathrm{C}_{21} \mathrm{H}_{24} \mathrm{~N}_{2} \mathrm{O}_{2} \mathrm{Na}(\mathrm{M}+\mathrm{Na})^{+} 359.1730$, observed 359.1733.

\section{$N$-(Phenylmethoxy)-2-methyl-2-(1-methyl-1H-indol-2-yl)propanamide (7)}<smiles>Cn1c(C(C)(C)C(=O)NOCc2ccccc2)cc2ccccc21</smiles>

Prepared in $88 \%$ yield $(107 \mathrm{mg}, 0.33 \mathrm{mmol})$ as a colorless oil from the reaction of 1methylindole (50 mg, $0.38 \mathrm{mmol})$ with 2-bromo-2-methyl- $N$-(phenylmethoxy)propanamide (0.13 g, 0.46) 1 via general procedure A. $R_{f}=0.43$ (6:4, hexanes: ethyl acetate); ${ }^{1} \mathrm{H}$ NMR (500 $\left.\mathrm{MHz}, \mathrm{CDCl}_{3}\right): \delta 8.07(\mathrm{~s}, 1 \mathrm{H}), 7.54(\mathrm{dd}, J=8.1,1.1 \mathrm{~Hz}, 1 \mathrm{H}), 7.29(\mathrm{dd}, J=8.3,1.0 \mathrm{~Hz}, 1 \mathrm{H}), 7.26$ - $7.17(\mathrm{~m}, 4 \mathrm{H}), 7.12$ (dd, $J=7.7,1.8 \mathrm{~Hz}, 2 \mathrm{H}), 7.08$ (td, $J=7.5,1.1 \mathrm{~Hz}, 1 \mathrm{H}), 6.88$ (s, 1H), 4.74 $(\mathrm{s}, 2 \mathrm{H}), 3.71(\mathrm{~s}, 3 \mathrm{H}), 1.64(\mathrm{~s}, 6 \mathrm{H}) ;{ }^{13} \mathrm{C} \mathrm{NMR}\left(101 \mathrm{MHz}, \mathrm{CDCl}_{3}\right)$ : 174.9, 137.7, 135.0, 129.2, $128.4,128.3,126.1,125.6,122.0,120.5,119.5,117.6,109.6,77.9,41.1,32.7$, 26.8; FT-IR (neat): 3250, 3059, 2972, 2933, 2876, 1661, 1545, 1465, 1424, 1371, 1361, 1328, $1229 \mathrm{~cm}^{-1}$; HRESI-MS: calculated for $\mathrm{C}_{20} \mathrm{H}_{22} \mathrm{~N}_{2} \mathrm{O}_{2} \mathrm{Na}(\mathrm{M}+\mathrm{Na})^{+}$345.1573, observed 345.1575. 
( \pm )-(3a $R, 8 \mathrm{a} S)-1-p h e n y l m e t h o x y-3,3,3 \mathrm{a}, 8$-tetramethyl-3,3a,8,8a-tetrahydropyrrolo[2,3b] indol-2(1H)-one (8)<smiles>CN1c2c(cccc2C(C)(C)C(=O)NOc2ccccc2)C[C@]1(C)N(Cc1ccccc1)Cc1ccccc1</smiles>

Prepared in $94 \%$ yield $(173 \mathrm{mg}, 0.33 \mathrm{mmol})$ as a colorless oil from the reaction of 1methylindole 4 (50 mg, $0.35 \mathrm{mmol}$ ) with 2-bromo-2-methyl- $N$-(phenylmethoxy)propanamide 1 (282 $\mathrm{mg}, 1.05 \mathrm{mmol})$ and $\mathrm{Na}_{2} \mathrm{CO}_{3}(110 \mathrm{mg}, 1.05 \mathrm{mmol})$ in HFIP $(0.25 \mathrm{M})$, via general procedure A with slight modification. OR, Prepared in $85 \%$ yield $(33 \mathrm{mg}, 0.063 \mathrm{mmol})$ as a colorless oil from the reaction of $5(25 \mathrm{mg}, 0.074 \mathrm{mmol})$ with 2-bromo-2-methyl- $N$ (phenylmethoxy)propanamide 1 (45 mg, $0.15 \mathrm{mmol}$ ) and $\mathrm{Na}_{2} \mathrm{CO}_{3}(16 \mathrm{mg}, 0.15)$ in HFIP $(0.25 \mathrm{M})$, via general procedure A with slight modification. $R_{f}=0.38$ (1:1, hexanes: ethyl acetate); ${ }^{1} \mathrm{H}$ NMR (500 MHz, $\left.\mathrm{CDCl}_{3}\right): \delta 7.61(\mathrm{~s}, 1 \mathrm{H}), 7.50-7.43(\mathrm{~m}, 2 \mathrm{H}), 7.43-7.36(\mathrm{~m}, 3 \mathrm{H})$, $7.33-7.27(\mathrm{~m}, 5 \mathrm{H}), 7.04(\mathrm{dd}, J=8.3,2.0 \mathrm{~Hz}, 1 \mathrm{H}), 6.85(\mathrm{~d}, J=2.0 \mathrm{~Hz}, 1 \mathrm{H}), 6.32(\mathrm{~d}, J=8.2 \mathrm{~Hz}$, $1 \mathrm{H}), 5.15(\mathrm{~d}, J=10.7 \mathrm{~Hz}, 1 \mathrm{H}), 5.00(\mathrm{~d}, J=10.7 \mathrm{~Hz}, 1 \mathrm{H}), 4.80(\mathrm{~s}, 2 \mathrm{H}), 4.29(\mathrm{~s}, 1 \mathrm{H}), 2.93$ (s, 3H), $1.50(\mathrm{~s}, 6 \mathrm{H}), 1.18(\mathrm{~s}, 3 \mathrm{H}), 1.15(\mathrm{~s}, 3 \mathrm{H}), 0.92$ (s, 3H). ${ }^{13} \mathrm{C} \mathrm{NMR}\left(101 \mathrm{MHz}, \mathrm{CDCl}_{3}\right): \delta 174.3$, 149.6, 135.1, 135.1, 133.2, 132.9, 129.8, 129.2, 129.1, 128.7, 128.6, 128.5, 126.3, 122.2, 106.9, 88.2, 77.9, 77.0, 51.4, 45.1, 43.6, 34.5, 27.2, 27.0, 24.3, 21.9, 21.9 ; FT-IR (neat): 3411, 2957 , 2868, 2830, 1680, 1657, 1622, 1582, 1514, 1482, 1453, 1438, 1418, 1381, 1362, 1308, 1261, $1208 \mathrm{~cm}^{-1}$; HRESI-MS: calculated for $\mathrm{C}_{32} \mathrm{H}_{37} \mathrm{~N}_{3} \mathrm{O}_{4} \mathrm{Na}(\mathrm{M}+\mathrm{Na})^{+}$550.2676, observed 550.2667. 
( \pm )-(3a $R, 8 \mathrm{a} S)$-1-Phenylmethoxy-3a-(propan-2-yl)-3,3,8-trimethyl-3,3a,8,8atetrahydropyrrolo[2,3-b]indol-2(1H)-one (10a)

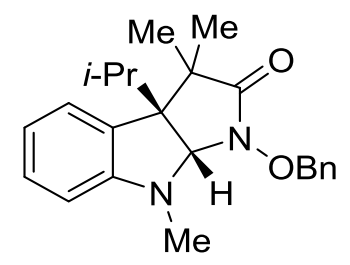

Prepared in 50\% yield (52 mg, $0.14 \mathrm{mmol}$ ) as a white solid from the reaction of 1-methyl-3isopropylindole $(50 \mathrm{mg}, 0.29 \mathrm{mmol})$ with 2-bromo-2-methyl- $N$-(phenylmethoxy)propanamide (78.3 mg, $0.29 \mathrm{mmol})$ via general procedure A. $R_{f}=0.58$ (7:3, hexanes: ethyl acetate); mp 107.2 - $108.6{ }^{\circ} \mathrm{C} ;{ }^{1} \mathrm{H}$ NMR $\left(500 \mathrm{MHz}, \mathrm{CDCl}_{3}\right): \delta 7.51-7.43(\mathrm{~m}, 2 \mathrm{H}), 7.43-7.33(\mathrm{~m}, 3 \mathrm{H}), 7.14(\mathrm{td}, J$ $=7.7,1.3 \mathrm{~Hz}, 1 \mathrm{H}), 6.98(\mathrm{dd}, J=7.4,1.3 \mathrm{~Hz}, 1 \mathrm{H}), 6.70$ (br.d, $J=7.5, \mathrm{~Hz}, 1 \mathrm{H}), 6.41$ (dd, $J=7.9$, $1.0 \mathrm{~Hz}, 1 \mathrm{H}), 5.18(\mathrm{~d}, J=10.2 \mathrm{~Hz}, 1 \mathrm{H}), 4.98(\mathrm{~d}, J=10.2 \mathrm{~Hz}, 1 \mathrm{H}), 4.61(\mathrm{~s}, 1 \mathrm{H}), 2.95(\mathrm{~s}, 3 \mathrm{H}), 2.23$ (hept, $J=6.7 \mathrm{~Hz}, 1 \mathrm{H}), 1.31(\mathrm{~s}, 3 \mathrm{H}), 1.15(\mathrm{~s}, 3 \mathrm{H}), 0.82$ (d, $J=6.7 \mathrm{~Hz}, 3 \mathrm{H}), 0.68$ (d, $J=6.7 \mathrm{~Hz}$, $3 \mathrm{H}) ;{ }^{13} \mathrm{C}$ NMR (101 MHz, $\left.\mathrm{CDCl}_{3}\right): \delta 175.0,151.2,135.1,129.5,128.9,128.5,128.5,128.1$, 125.8, 117.6, 106.9, 83.7, 77.1, 59.3, 44.2, 34.3, 30.9, 25.8, 21.5, 19.1, 17.7; FT-IR (neat): 3031, 2986, 2933, 2874, 1704, 1604, 1492, 1455, 1429, 1383, 1363, 1336, 1307, 1278, 1251, $1212 \mathrm{~cm}^{-}$ ${ }^{1}$; HRESI-MS: calculated for $\mathrm{C}_{23} \mathrm{H}_{28} \mathrm{~N}_{2} \mathrm{O}_{2} \mathrm{Na}(\mathrm{M}+\mathrm{Na})^{+}$387.2043, observed 387.2049.

( \pm )-(3aR,8aS)-1-phenylmethoxy-5-methoxy-3,3,8-trimethyl-3a-(propan-2-yl)-3,3a,8,8atetrahydropyrrolo[2,3-b]indol-2(1H)-one $(10 b)$<smiles>COc1ccc2c(c1)[C@@]1(F)[C@@H](N(Cc3ccccc3)C(=O)C1(C)C)N2C</smiles>

Prepared in 63\% yield (73 g, $0.18 \mathrm{mmol}$ ) as a colorless oil from the reaction of 1-methyl-5methoxy-3-isopropylindole $\quad(60 \mathrm{mg}, \quad 0.29 \mathrm{mmol})$ with 2-bromo-2-methyl- $N$ (phenylmethoxy)propanamide $(96 \mathrm{mg}, 0.35 \mathrm{mmol})$ via general procedure A. $R_{f}=0.55(3: 1$, hexanes: ethyl acetate); ${ }^{1} \mathrm{H} \mathrm{NMR}\left(500 \mathrm{MHz}, \mathrm{CDCl}_{3}\right): \delta 7.51-7.45(\mathrm{~m}, 2 \mathrm{H}), 7.44-7.32(\mathrm{~m}, 3 \mathrm{H})$, $6.71(\mathrm{dd}, J=8.5,2.6 \mathrm{~Hz}, 1 \mathrm{H}), 6.60(\mathrm{~d}, J=2.6 \mathrm{~Hz}, 1 \mathrm{H}), 6.35(\mathrm{~d}, J=8.5 \mathrm{~Hz}, 1 \mathrm{H}), 5.18(\mathrm{~d}, J=$ $10.2 \mathrm{~Hz}, 1 \mathrm{H}), 5.00$ (d, $J=10.2 \mathrm{~Hz}, 1 \mathrm{H}), 4.60$ (s, 1H), 3.75 (s, 3H), 2.91 (s, 3H), 2.24 (hept, $J=$ $6.7 \mathrm{~Hz}, 1 \mathrm{H}), 1.29(\mathrm{~s}, 3 \mathrm{H}), 1.11(\mathrm{~s}, 3 \mathrm{H}), 0.80(\mathrm{~d}, J=6.7 \mathrm{~Hz}, 3 \mathrm{H}), 0.73(\mathrm{~d}, J=6.6 \mathrm{~Hz}, 3 \mathrm{H}) ;{ }^{13} \mathrm{C}$ 
NMR (126 MHz, $\left.\mathrm{CDCl}_{3}\right): \delta 175.1,152.7,146.1,135.1,130.1,129.5,128.8,128.5,113.1,112.6$, 107.5, 84.3, 77.3, 59.4, 56.0, 44.1, 35.6, 30.7, 26.2, 20.8, 19.3, 17.8; FT-IR (neat): 3063, 3029, 2972, 2934, 1700, 1621, 1594, 1502, 1464, 1452, 1431, 1394, 1382, 1376, 1362, 1338, 1327, 1282, 1260, 1250, $1216 \mathrm{~cm}^{-1}$; HRESI-MS: calculated for $\mathrm{C}_{24} \mathrm{H}_{30} \mathrm{~N}_{2} \mathrm{O}_{3} \mathrm{Na}(\mathrm{M}+\mathrm{Na})^{+}$417.2148, observed 417.2165.

( \pm )-(3aR,8aS)-1-Phenylmethoxy-3a-cyclohexyl-3,3,8-trimethyl-3,3a,8,8atetrahydropyrrolo[2,3-b]indol-2(1H)-one (10c)<smiles>CN1c2ccccc2C2(CCCCC2)C1(C)C(=O)N(Cc1ccccc1)OCc1ccccc1</smiles>

Prepared in 57\% yield $(60 \mathrm{mg}, 0.15 \mathrm{mmol})$ as a colorless oil from the reaction of 3-cyclohexyl-1methylindole (55 mg, $0.26 \mathrm{mmol}$ ) with 2-bromo-2-methyl- $N$-(phenylmethoxy)propanamide (70.2 $\mathrm{mg}, 0.26 \mathrm{mmol})$ via general procedure A. $R_{f}=0.6$ (8:2, hexanes: ethyl acetate); ${ }^{1} \mathrm{H}$ NMR (500 $\left.\mathrm{MHz}, \mathrm{CDCl}_{3}\right): \delta 7.52-7.45(\mathrm{~m}, 2 \mathrm{H}), 7.43-7.33(\mathrm{~m}, 3 \mathrm{H}), 7.14(\mathrm{ddd}, J=7.9,7.5,1.3 \mathrm{~Hz}, 1 \mathrm{H})$, 6.94 (ddd, $J=7.4,1.3,0.5 \mathrm{~Hz}, 1 \mathrm{H}), 6.71$ (td, $J=7.4,1.0 \mathrm{~Hz}, 1 \mathrm{H}$ ), 6.42 (br.d, $J=7.8, \mathrm{~Hz}, 1 \mathrm{H}$ ), 5.19 (d, $J=10.3 \mathrm{~Hz}, 1 \mathrm{H}), 4.99$ (dd, $J=10.3,0.6 \mathrm{~Hz}, 1 \mathrm{H}), 4.67$ (s, 1H), 2.94 (s, 3H), 1.83 (tt, $J=$ 11.7, $2.7 \mathrm{~Hz}, 1 \mathrm{H}), 1.71-1.49$ (m, 5H), 1.29 (s, 3H), 1.20 (qdd, $J=12.6,3.7,3.3 \mathrm{~Hz}, 2 \mathrm{H}), 1.09$ (s, 3H), 0.99 (qt, $J=12.6,3.2 \mathrm{~Hz}, 1 \mathrm{H}$ ), 0.92 (qd, $J=12.8,3.8 \mathrm{~Hz}, 1 \mathrm{H}), 0.61$ (qd, $J=13.0,3.5$ $\mathrm{Hz}, 1 \mathrm{H}) ;{ }^{13} \mathrm{C}$ NMR (101 MHz, $\left.\mathrm{CDCl}_{3}\right): 175.4,151.4,135.2,129.5,128.9,128.5,128.4,128.4$, 125.4, 117.7, 107.1, 84.2, 77.3, 59.3, 44.1, 41.7, 34.6, 29.0, 27.9, 26.4, 26.3, 26.2, 26.2, 20.8; FT-IR (neat): 3031, 2922, 2851, 1703, 1603, 1489, 1453, 1428, 1382, 1362, 1311, 1276, 1233 $\mathrm{cm}^{-1}$; HRESI-MS: calculated for $\mathrm{C}_{26} \mathrm{H}_{33} \mathrm{~N}_{2} \mathrm{O}_{2}(\mathrm{M}+\mathrm{H})^{+}$405.2536, observed 405.2536. 


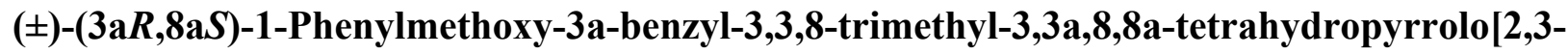
b]indol-2(1H)-one (10d)<smiles>CN1c2ccccc2C2(Br)[C@H]1N(OCc1ccccc1)C(=O)C2(C)C</smiles>

Prepared in $61 \%$ yield $(61 \mathrm{mg}, 0.15 \mathrm{mmol})$ as a white solid from the reaction of 3-benzyl-1methylindole (53 mg, $0.24 \mathrm{mmol}$ ) with 2-bromo-2-methyl- $N$-(phenylmethoxy)propanamide (65 $\mathrm{mg}, 0.24 \mathrm{mmol})$ via general procedure A. $R_{f}=0.47$ (7:3, hexanes: ethyl acetate); mp $88.5-90.9$ ${ }^{\circ} \mathrm{C} ;{ }^{1} \mathrm{H}$ NMR $\left(500 \mathrm{MHz}, \mathrm{CDCl}_{3}\right): \delta 7.47$ - $7.43(\mathrm{~m}, 2 \mathrm{H}), 7.42$ - 7.37 (m, 3H), 7.14 (ddd, $J=7.4$, 1.3, $0.6 \mathrm{~Hz}, 1 \mathrm{H}), 7.08(\mathrm{td}, J=7.7,1.2 \mathrm{~Hz}, 1 \mathrm{H}), 7.12-6.97(\mathrm{~m}, 3 \mathrm{H}), 6.75(\mathrm{td}, J=7.5,1.0 \mathrm{~Hz}$, 1H), $6.54-6.44$ (m, 2H), 6.17 (br.d, $J=7.9 \mathrm{~Hz}, 1 \mathrm{H}), 5.17$ (d, $J=10.6 \mathrm{~Hz}, 1 \mathrm{H}), 4.92$ (d, $J=10.6$ $\mathrm{Hz}, 1 \mathrm{H}), 4.56(\mathrm{~s}, 1 \mathrm{H}), 3.02(\mathrm{~d}, J=13.3 \mathrm{~Hz}, 1 \mathrm{H}), 2.76$ (d, J=13.4 Hz, 1H), 2.41 (s, 3H), 1.39 (s, 3H), $1.22(\mathrm{~s}, 3 \mathrm{H}) ;{ }^{13} \mathrm{C} \mathrm{NMR}\left(101 \mathrm{MHz}, \mathrm{CDCl}_{3}\right): \delta 173.8,151.3,136.6,135.3,130.0,129.7$, $129.5,128.9,128.8,128.6,127.5,126.4,125.9,117.7,107.5,84.1,77.2,57.1,44.4,40.0,34.2$, 24.5, 23.2; FT-IR (neat): 3061, 3026, 2987, 2968, 2940, 2805, 1705, 1603, 1495, 1470, 1454, 1429, 1401, 1391, 1364, 1310, 1285, 1252, 1223, $1212 \mathrm{~cm}^{-1}$; HRESI-MS: calculated for $\mathrm{C}_{27} \mathrm{H}_{28} \mathrm{~N}_{2} \mathrm{O}_{2} \mathrm{Na}(\mathrm{M}+\mathrm{Na})^{+} 435.2043$, observed 435.2051.

( \pm )-(3aS,8aS)-1-Phenylmethoxy-3,3,8-trimethyl-3a-[2-[[1,1dimethylethyl)dimethylsilyl $]$ oxy] ethyl]-3,3a,8,8a-tetrahydropyrrolo $[2,3-b]$ indol-2(1H)-one (10e)<smiles>CN1c2ccccc2C2(CCOC(C)(C)C)[C@@H]1N(OCc1ccccc1)C(=O)C2(C)C</smiles>

Prepared in $73 \%$ yield $(60 \mathrm{mg}, 0.13 \mathrm{mmol})$ as a colorless oil from the reaction of 3-[2-[[(1,1dimethylethyl)dimethylsilyl]oxy]ethyl]-1-methyl-1H-Indole (50 mg, $0.17 \mathrm{mmol})$ with 2-bromo2-methyl- $N$-(phenylmethoxy)propanamide $(56 \mathrm{mg}, 0.20 \mathrm{mmol})$ via general procedure A. $R_{f}=$ 0.46 (8:2, hexanes: ethyl acetate); ${ }^{1} \mathrm{H}$ NMR (500 MHz, $\left.\mathrm{CDCl}_{3}\right): 7.48-7.46$ (m, 2H), $7.41-7.36$ $(\mathrm{m}, 3 \mathrm{H}), 7.12(\mathrm{td}, J=7.7,1.3 \mathrm{~Hz}, 1 \mathrm{H}), 6.90(\mathrm{dd}, J=7.6,1.0 \mathrm{~Hz}, 1 \mathrm{H}), 6.69$ (br.d, $J=7.5, \mathrm{~Hz}$, 
1H), $6.40(\mathrm{~d}, J=7.7 \mathrm{~Hz}, 1 \mathrm{H}), 5.14(\mathrm{~d}, J=10.5 \mathrm{~Hz}, 1 \mathrm{H}), 4.99(\mathrm{~d}, J=10.5 \mathrm{~Hz}, 1 \mathrm{H}), 4.82(\mathrm{~s}, 1 \mathrm{H})$, $3.28(\mathrm{dd}, J=7.2,6.2 \mathrm{~Hz}, 2 \mathrm{H}), 2.94(\mathrm{~s}, 3 \mathrm{H}), 1.96(\mathrm{dt}, J=13.9,6.3 \mathrm{~Hz}, 1 \mathrm{H}), 1.78(\mathrm{dt}, J=14.1,7.2$ $\mathrm{Hz}, 1 \mathrm{H}), 1.23(\mathrm{~s}, 3 \mathrm{H}), 1.07(\mathrm{~s}, 3 \mathrm{H}), 0.81(\mathrm{~s}, 9 \mathrm{H}),-0.09(\mathrm{~s}, 3 \mathrm{H}),-0.10(\mathrm{~s}, 3 \mathrm{H}) ;{ }^{13} \mathrm{C}$ NMR $(126$ $\left.\mathrm{MHz}, \mathrm{CDCl}_{3}\right): \delta 174.1,151.0,135.1,129.6,129.0,128.9,128.7,128.6,124.9,117.9,107.0$, 85.0, 76.9, 60.0, 54.3, 44.2, 36.5, 34.4, 25.9, 24.2, 22.5, 18.2, -5.4; FT-IR (neat): 3031, 2951, 2927, 2881, 2855, 1708, 1605, 1490, 1471, 1461, 1428, 1384, 1362, 1282, 1252, $1212 \mathrm{~cm}^{-1}$; HRESI-MS: calculated for $\mathrm{C}_{28} \mathrm{H}_{41} \mathrm{~N}_{2} \mathrm{O}_{3} \mathrm{Si}(\mathrm{M}+\mathrm{H})^{+} 481.2881$, observed 481.2879.

\section{( \pm )-2-\{2-[-(3aS,8aS)-1-Phenylmethoxy-3,3,8-trimethyl-2-oxo-3,3a,8,8a-} tetrahydropyrrolo[2,3-b]indol-3a(1H)-yl]ethyl $\}-1 H$-Isoindole-1,3(2H)-dione (10f)<smiles>CN1c2ccccc2C2(CCNc3ccccc3)[C@H]1N(OCc1ccccc1)C(=O)C2(C)C</smiles>

Prepared in $87 \%$ yield $(73 \mathrm{mg}, 0.15 \mathrm{mmol})$ as a pale yellow solid from the reaction of 2-[2-(1methyl-1H-indol-3-yl)ethyl]-isoindole-1,3(2H)-dione (53 mg, $0.17 \mathrm{mmol})$ with 2-bromo-2methyl- $N$-(phenylmethoxy)propanamide $(57 \mathrm{mg}, 0.20 \mathrm{mmol})$ via general procedure A. $R_{f}=0.34$ (2:1, hexanes: ethyl acetate); mp $65.3-68.2^{\circ} \mathrm{C} ; 1 \mathrm{H} \mathrm{NMR} \mathrm{(500} \mathrm{MHz,} \mathrm{CDCl3):} \delta 7.73-7.69$ (m, 2H), $7.67-7.62(\mathrm{~m}, 2 \mathrm{H}), 7.53-7.51(\mathrm{~m}, 2 \mathrm{H}), 7.44-7.27(\mathrm{~m}, 3 \mathrm{H}), 6.96-6.92(\mathrm{~m}, 2 \mathrm{H}), 6.51$ (br.d, $J=7.5, \mathrm{~Hz}, 1 \mathrm{H}), 6.35$ (d, $J=7.8 \mathrm{~Hz}, 1 \mathrm{H}), 5.21$ (d, $J=10.6 \mathrm{~Hz}, 1 \mathrm{H}), 5.04$ (d, $J=10.5 \mathrm{~Hz}$, 1H), $4.86(\mathrm{~s}, 1 \mathrm{H}), 3.37$ (ddd, $J=13.8,9.5,4.0 \mathrm{~Hz}, 1 \mathrm{H}), 3.23$ (ddd, $J=14.0,8.8,7.4 \mathrm{~Hz}, 1 \mathrm{H})$, 3.00 (s, 3H), 2.32 (ddd, $J=13.8,9.6,7.5 \mathrm{~Hz}, 1 \mathrm{H}), 1.68$ (ddd, $J=13.4,8.8,4.0 \mathrm{~Hz}, 1 \mathrm{H}), 1.20$ (s, 3H), $0.98(\mathrm{~s}, 3 \mathrm{H}) ;{ }^{13} \mathrm{C} \mathrm{NMR}\left(126 \mathrm{MHz}, \mathrm{CDCl}_{3}\right): \delta 174.0,167.9,151.1,135.1,133.8,131.9$, $129.8,129.1,128.7,128.7,127.9,124.4,123.0,118.2,107.2,84.6,77.0,54.0,44.5,34.5,34.3$, 31.5, 24.5, 21.5; FT-IR (neat): 3059, 3028, 2932, 2869, 1770, 1704, 1605, 1489, 1454, 1398, 1370, 1285, $1212 \mathrm{~cm}^{-1}$; HRESI-MS: calculated for $\mathrm{C}_{30} \mathrm{H}_{30} \mathrm{~N}_{3} \mathrm{O}_{4}(\mathrm{M}+\mathrm{H})^{+}$496.2231, observed 496.2235 . 
( \pm )-Methyl [(3aR,8aS)-1-phenylmethoxy-3,3,8-trimethyl-2-oxo-2,3,8,8atetrahydropyrrolo[2,3-b]indol-3a(1H)-yl]acetate $(10 \mathrm{~g})$<smiles>COC(=O)CC12c3ccccc3N(C)C1(C)C(C)(C)C(=O)N2OCc1ccccc1</smiles>

Prepared in $83 \%$ yield $(160 \mathrm{mg}, 0.41 \mathrm{mmol})$ as a colorless oil from the reaction of methyl 1methylindole-3-acetate $(0.1 \mathrm{~g}, \quad 0.49 \mathrm{mmol}) \quad$ with 2-bromo-2-methyl- $N$ (phenylmethoxy)propanamide $(160 \mathrm{mg}, 0.59 \mathrm{mmol})$ via general procedure A. $R_{f}=0.6(1: 1$, hexanes: ethyl acetate); ${ }^{1} \mathrm{H}$ NMR $\left(500 \mathrm{MHz}, \mathrm{CDCl}_{3}\right): \delta 7.49-7.47(\mathrm{~m}, 2 \mathrm{H}), 7.40-7.35(\mathrm{~m}, 3 \mathrm{H})$, $7.12(\mathrm{td}, J=7.7,1.3 \mathrm{~Hz}, 1 \mathrm{H}), 6.93(\mathrm{dd}, J=7.4,1.0 \mathrm{~Hz}, 1 \mathrm{H}), 6.67(\mathrm{td}, J=7.4,1.0 \mathrm{~Hz}, 1 \mathrm{H}), 6.42$ (br.d, $J=7.8 \mathrm{~Hz}, 1 \mathrm{H}), 5.21$ (d, $J=10.4 \mathrm{~Hz}, 1 \mathrm{H}), 5.03$ (d, $J=10.4 \mathrm{~Hz}, 1 \mathrm{H}), 5.01(\mathrm{~s}, 1 \mathrm{H}), 3.42$ (s, 3H), $2.96(\mathrm{~s}, 3 \mathrm{H}), 2.79(\mathrm{~d}, J=15.4 \mathrm{~Hz}, 1 \mathrm{H}), 2.44$ (d, $J=15.4 \mathrm{~Hz}, 1 \mathrm{H}), 1.22(\mathrm{~s}, 3 \mathrm{H}), 1.04$ (s, 3H); ${ }^{13} \mathrm{C}$ NMR $\left(101 \mathrm{MHz}, \mathrm{CDCl}_{3}\right): \delta 173.7,170.7,151.4,135.0,129.7,129.0,128.9,128.6,128.5$, 124.4, 118.0, 107.3, 85.8, 77.0, 53.2, 51.5, 44.0, 39.1, 35.1, 25.1, 21.9; FT-IR (neat): 3031, 2975, 2949, 2877, 1705, 1605, 1489, 1454, 1434, 1411, 1383, 1366, 1325, 1306, $1283 \mathrm{~cm}^{-1}$; HRESIMS: calculated for $\mathrm{C}_{23} \mathrm{H}_{26} \mathrm{~N}_{2} \mathrm{O}_{4} \mathrm{Na}(\mathrm{M}+\mathrm{Na})^{+} 417.1785$, observed 417.1789.

( \pm )-[(3aR,8aS)-1-Phenylmethoxy-3,3,8-trimethyl-2-oxo-2,3,8,8a-tetrahydropyrrolo[2,3b] indol-3a(1H)-yl]acetonitrile (10h)<smiles>CN1c2ccccc2C2(CC#N)N1N(Cc1ccccc1)C(=O)C2(C)C</smiles>

Prepared in $81 \%$ yield $(106 \mathrm{mg}, 0.24 \mathrm{mmol})$ as a colorless oil from the reaction of 1-methylindole-3-acetonitrile $\quad(50 \quad \mathrm{mg}, \quad 0.29 \quad \mathrm{mmol})$ with 2-bromo-2-methyl- $N$ (phenylmethoxy)propanamide $(95.3 \mathrm{mg}, 0.35 \mathrm{mmol})$ via general procedure A. $R_{f}=0.42(7: 3$, hexanes: ethyl acetate); ${ }^{1} \mathrm{H} \mathrm{NMR}\left(500 \mathrm{MHz}, \mathrm{CDCl}_{3}\right): \delta 7.49-7.45(\mathrm{~m}, 2 \mathrm{H}), 7.44-7.38(\mathrm{~m}, 3 \mathrm{H})$, $7.21(\mathrm{td}, J=7.7,1.2 \mathrm{~Hz}, 1 \mathrm{H}), 7.14(\mathrm{ddd}, J=7.5,1.2,0.5 \mathrm{~Hz}, 1 \mathrm{H}), 6.77(\mathrm{td}, J=7.5,1.0 \mathrm{~Hz}, 1 \mathrm{H})$, 6.48 (br.d, $J=7.9 \mathrm{~Hz}, 1 \mathrm{H}), 5.21$ (d, $J=10.6 \mathrm{~Hz}, 1 \mathrm{H}), 5.00$ (d, $J=10.6 \mathrm{~Hz}, 1 \mathrm{H}), 4.53$ (s, 1H), 
2.99 (s, 3H), 2.68 (d, $J=16.6 \mathrm{~Hz}, 1 \mathrm{H}), 2.39$ (d, $J=16.7 \mathrm{~Hz}, 1 \mathrm{H}), 1.29$ (s, 3H), 1.18 (s, 3H); ${ }^{13} \mathrm{C}$ NMR (101 MHz, $\left.\mathrm{CDCl}_{3}\right): \delta 172.7,150.2,134.8,130.0,129.8,129.3,128.7,127.3,124.8,118.8$, 116.8, 107.9, 85.3, 77.1, 52.7, 43.7, 34.2, 24.0, 24.0, 22.2; FT-IR (neat): 3056, 3032, 2975, 2936, 2877, 2251, 1704, 1604, 1489, 1471, 1455, 1428, 1385, 1366, 1314, 1282, 1250, $1212 \mathrm{~cm}^{-1}$; HRESI-MS: calculated for $\mathrm{C}_{22} \mathrm{H}_{23} \mathrm{~N}_{3} \mathrm{O}_{2} \mathrm{Na}(\mathrm{M}+\mathrm{Na})^{+}$384.1682, observed 384.1697.

\section{( \pm )-(3aR,8aS)-1-Phenylmethoxy-5-bromo-3,3,3a,8-tetramethyl-3,3a,8,8a-} tetrahydropyrrolo $[2,3-b]$ indol-2(1H)-one $(10 \mathrm{i})$<smiles>CN1c2ccc(Br)cc2C2(C)[C@@H]1N(Cc1ccccc1)C(=O)C2(C)C</smiles>

Prepared in $80 \%$ yield $(77 \mathrm{mg}, 0.18 \mathrm{mmol})$ as a colorless oil from the reaction of 5-bromo-1,3dimethylindole $(50 \mathrm{mg}, 0.22 \mathrm{mmol})$ with 2-bromo-2-methyl- $N$-(phenylmethoxy)propanamide (61 mg, $0.22 \mathrm{mmol}$ ) via general procedure A. $R_{f}=0.33$ (8:2, hexanes: ethyl acetate); ${ }^{1} \mathrm{H}$ NMR $\left(500 \mathrm{MHz}, \mathrm{CDCl}_{3}\right): \delta 7.49-7.43(\mathrm{~m}, 2 \mathrm{H}), 7.43-7.34(\mathrm{~m}, 3 \mathrm{H}), 7.21(\mathrm{dd}, J=8.3,2.0 \mathrm{~Hz}, 1 \mathrm{H})$, $7.01(\mathrm{~d}, J=1.9 \mathrm{~Hz}, 1 \mathrm{H}), 6.28(\mathrm{~d}, J=8.4 \mathrm{~Hz}, 1 \mathrm{H}), 5.16(\mathrm{~d}, J=10.7 \mathrm{~Hz}, 1 \mathrm{H}), 5.00(\mathrm{~d}, J=10.6 \mathrm{~Hz}$, $1 \mathrm{H}), 4.30(\mathrm{~s}, 1 \mathrm{H}), 2.91(\mathrm{~s}, 3 \mathrm{H}), 1.19(\mathrm{~s}, 3 \mathrm{H}), 1.18(\mathrm{~s}, 3 \mathrm{H}), 1.02(\mathrm{~s}, 3 \mathrm{H}) ;{ }^{13} \mathrm{C} \mathrm{NMR}(126 \mathrm{MHz}$, $\left.\mathrm{CDCl}_{3}\right): \delta 174.2,149.6,135.0,134.4,131.2,129.8,129.1,128.6,127.0,109.9,108.5,88.2,77.1$, 51.4, 43.6, 34.5, 24.3, 21.9, 21.6; FT-IR (neat): 3062, 3031, 2972, 2933, 2873, 1703, 1599, 1485, 1454, 1429, 1413, 1372, 1305, 1270, 1239, $1209 \mathrm{~cm}^{-1}$; HRESI-MS: calculated for $\mathrm{C}_{21} \mathrm{H}_{23} \mathrm{~N}_{2} \mathrm{O}_{2} \mathrm{BrNa}(\mathrm{M}+\mathrm{Na})^{+} 437.0835$, observed 437.0843 . 
$( \pm)-(3 a R, 8 a S)-1-P h e n y l m e t h o x y-8-b e n z y l-5-b r o m o-3,3,3 a, 8-t e t r a m e t h y l-3,3 a, 8,8 a-$ tetrahydropyrrolo[2,3-b]indol-2(1H)-one $(10 \mathrm{j})$<smiles>CC1(C)C(=O)N(OCc2ccccc2)[C@H]2c3cc(Br)ccc3N(Cc3ccccc3)C21C</smiles>

Prepared in $85 \%$ yield $(71 \mathrm{mg}, 0.14 \mathrm{mmol})$ as a colorless oil from the reaction of 1-benzyl-5bromo-3-methylindole $\quad(50 \mathrm{mg}, \quad 0.17 \mathrm{mmol})$ with 2-bromo-2-methyl- $N$ (phenylmethoxy)propanamide $(55 \mathrm{mg}, 0.20 \mathrm{mmol})$ via general procedure A. $R_{f}=0.34(8: 2$, hexanes: ethyl acetate); ${ }^{1} \mathrm{H} \mathrm{NMR}\left(500 \mathrm{MHz}, \mathrm{CDCl}_{3}\right): \delta 7.38-7.29(\mathrm{~m}, 8 \mathrm{H}), 7.25-7.23(\mathrm{~m}, 2 \mathrm{H})$, $7.14(\mathrm{dd}, J=8.4,2.0 \mathrm{~Hz}, 1 \mathrm{H}), 7.06(\mathrm{~d}, J=2.1 \mathrm{~Hz}, 1 \mathrm{H}), 6.21(\mathrm{~d}, J=8.4 \mathrm{~Hz}, 1 \mathrm{H}), 5.13(\mathrm{~d}, J=$ $10.5 \mathrm{~Hz}, 1 \mathrm{H}), 4.92(\mathrm{~d}, J=10.6 \mathrm{~Hz}, 1 \mathrm{H}), 4.60(\mathrm{~d}, J=16.1 \mathrm{~Hz}, 1 \mathrm{H}), 4.54(\mathrm{~s}, 1 \mathrm{H}), 4.42(\mathrm{~d}, J=16.1$ $\mathrm{Hz}, 1 \mathrm{H}), 1.21$ (s, 3H), 1.19 (s, 3H), 1.11 (s, 3H).; ${ }^{13} \mathrm{C} \mathrm{NMR}\left(126 \mathrm{MHz}, \mathrm{CDCl}_{3}\right): \delta 174.1,148.7$, $137.4,134.8,134.3,131.2,129.7,129.0,128.7,128.5,127.4,127.2,127.1,109.9,108.9,85.9$, 77.0, 51.6, 51.2, 43.6, 24.4, 21.8, 21.8; FT-IR (neat): 3062, 3030, 2972, 2931, 2872, 1705, 1597 , 1483, 1452, 1414, 1376, 1351, 1310, 1265, 1238, $1217 \mathrm{~cm}^{-1}$; HRESI-MS: calculated for $\mathrm{C}_{27} \mathrm{H}_{27} \mathrm{~N}_{2} \mathrm{O}_{2} \mathrm{BrNa}(\mathrm{M}+\mathrm{Na})^{+}$513.1148, observed 513.1140.

( \pm )-(3aR,8aS)-1-Phenylmethoxy-8-benzyl-3,3,3a-trimethyl-3,3a,8,8a-tetrahydropyrrolo[2,3b]indol-2(1H)-one (10k)<smiles></smiles>

Prepared in 76\% yield $(70.8 \mathrm{mg}, 0.17 \mathrm{mmol})$ as a colorless oil from the reaction of 1-benzyl-3methylindole (50 mg, $0.23 \mathrm{mmol}$ ) with 2-bromo-2-methyl- $N$-(phenylmethoxy)propanamide (61.3 $\mathrm{mg}, 0.23 \mathrm{mmol})$ via general procedure A. $R_{f}=0.56$ (7:3, hexanes: ethyl acetate); ${ }^{1} \mathrm{H}$ NMR (500 $\left.\mathrm{MHz}, \mathrm{CDCl}_{3}\right): \delta 7.37-7.25(\mathrm{~m}, 10 \mathrm{H}), 7.04(\mathrm{td}, J=7.7,1.2 \mathrm{~Hz}, 1 \mathrm{H}), 6.98(\mathrm{ddd}, J=7.4,1.3,0.6$ $\mathrm{Hz}, 1 \mathrm{H}), 6.68$ (td, $J=7.4,0.9 \mathrm{~Hz}, 1 \mathrm{H}), 6.35$ (br.d, $J=7.9 \mathrm{~Hz}, 1 \mathrm{H}), 5.12$ (d, $J=10.5 \mathrm{~Hz}, 1 \mathrm{H})$, $4.90(\mathrm{~d}, J=10.5 \mathrm{~Hz}, 1 \mathrm{H}), 4.63(\mathrm{~d}, J=15.8 \mathrm{~Hz}, 1 \mathrm{H}), 4.56(\mathrm{~s}, 1 \mathrm{H}), 4.43(\mathrm{~d}, J=16.0 \mathrm{~Hz}, 1 \mathrm{H}), 1.21$ (s, 3H), 1.21 (s, 3H), 1.08 (s, 3H); ${ }^{13} \mathrm{C} \mathrm{NMR}\left(101 \mathrm{MHz}, \mathrm{CDCl}_{3}\right): \delta 174.5,149.8,138.0,134.9$, 
$132.0,129.7,128.9,128.6,128.5,128.5,127.2,127.2,124.1,118.3,107.5,86.1,76.9,51.6,51.5$, 43.8, 24.4, 21.9, 21.8; FT-IR (neat): 3060, 3030, 2972, 2930, 2871, 1703, 1603, 1485, 1452, 1394, 1375, 1352, 1310, 1267, 1239, $1216 \mathrm{~cm}^{-1}$; HRESI-MS: calculated for $\mathrm{C}_{27} \mathrm{H}_{28} \mathrm{~N}_{2} \mathrm{O}_{2} \mathrm{Na}$ $(\mathrm{M}+\mathrm{Na})^{+}$435.2043, observed 435.2044.

( \pm )-(3aR,8aS)-1-Phenylmethoxy-8-benzyl-3a-cyclohexyl-3,3-dimethyl-3,3a,8,8atetrahydropyrrolo[2,3-b]indol-2(1H)-one (10I)<smiles>CCCCC12c3ccccc3N(Cc3ccccc3)[C@@H]1N(Cc1ccccc1)C(=O)C2(C)C</smiles>

Prepared in $57 \%$ yield $(71.3 \mathrm{~g}, 0.16 \mathrm{mmol})$ as a white solid from the reaction of 1-benzyl-3cyclohexylindole (82 mg, $0.28 \mathrm{mmol}$ ) with 2-bromo-2-methyl- $N$-(phenylmethoxy)propanamide $77 \mathrm{mg}, 0.28 \mathrm{mmol})$ via general procedure A. $R_{f}=0.66$ (7:3, hexanes: ethyl acetate); mp $61.1-$ $63.3{ }^{\circ} \mathrm{C} ;{ }^{1} \mathrm{H}$ NMR $\left(500 \mathrm{MHz}, \mathrm{CDCl}_{3}\right): \delta 7.34-7.25(\mathrm{~m}, 10 \mathrm{H}), 7.06(\mathrm{td}, J=7.7,1.3 \mathrm{~Hz}, 1 \mathrm{H})$, $6.93(\mathrm{ddd}, J=7.4,1.3,0.5 \mathrm{~Hz}, 1 \mathrm{H}), 6.72(\mathrm{td}, J=7.4,1.0 \mathrm{~Hz}, 1 \mathrm{H}), 6.37$ (br.d $J=8.0, \mathrm{~Hz}, 1 \mathrm{H})$, $5.11(\mathrm{~d}, J=10.0 \mathrm{~Hz}, 1 \mathrm{H}), 4.93(\mathrm{~s}, 1 \mathrm{H}), 4.83(\mathrm{~d}, J=10.1 \mathrm{~Hz}, 1 \mathrm{H}), 4.64(\mathrm{~d}, J=16.3 \mathrm{~Hz}, 1 \mathrm{H}), 4.45$ $(\mathrm{d}, J=16.3 \mathrm{~Hz}, 1 \mathrm{H}), 1.86(\mathrm{tt}, J=11.9,2.7 \mathrm{~Hz}, 1 \mathrm{H}), 1.73-1.55(\mathrm{~m}, 4 \mathrm{H}), 1.53-1.40(\mathrm{~m}, 1 \mathrm{H})$, 1.28 (s, 3H), 1.18 (dddq, $J=15.5,9.4,6.0,3.5 \mathrm{~Hz}, 2 \mathrm{H}), 1.08$ (s, 3H), 0.95 (qt, $J=13.2,3.4 \mathrm{~Hz}$, $1 \mathrm{H}), 0.74(\mathrm{qd}, J=12.7,3.3 \mathrm{~Hz}, 1 \mathrm{H}), 0.63(\mathrm{qd}, J=12.8,3.0 \mathrm{~Hz}, 1 \mathrm{H}) ;{ }^{13} \mathrm{C} \mathrm{NMR}(101 \mathrm{MHz}$, $\left.\mathrm{CDCl}_{3}\right): \delta 176.1,151.2,137.9,135.1,129.4,128.8,128.6,128.6,128.4,128.3,127.3,127.2$, $124.8,118.1,107.6,82.3,77.0,59.3,52.3,44.0,41.4,29.2,28.1,27.0,26.5,26.2,26.1,20.0$; FT-IR (neat): 3030, 2923, 2851, 1708, 1602, 1485, 1463, 1452, 1388, 1352, 1301, 1263, 1238, $1212 \mathrm{~cm}^{-1}$; HRESI-MS: calculated for $\mathrm{C}_{32} \mathrm{H}_{36} \mathrm{~N}_{2} \mathrm{O}_{2} \mathrm{Na}(\mathrm{M}+\mathrm{Na})^{+}$503.2669, observed 503.2678. 


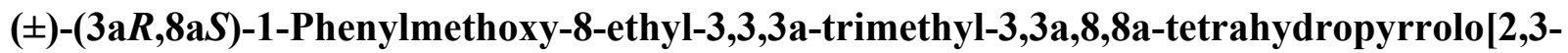
b]indol-2(1H)-one (10m)<smiles>CCN1c2ccccc2[C@]2(C)[C@@H]1N(Cc1ccccc1)C(=O)C2(C)C</smiles>

Prepared in $79 \%$ yield $(108 \mathrm{mg}, 0.31 \mathrm{mmol})$ as a colorless oil from the reaction of 1-ethyl-3methylindole (62 mg, $0.39 \mathrm{mmol}$ ) with 2-bromo-2-methyl- $N$-(phenylmethoxy)propanamide (105 mg, 0.39 mmol) via general procedure A. $R_{f}=0.30$ (8:2, hexanes: ethyl acetate); ${ }^{1} \mathrm{H}$ NMR (500 $\left.\mathrm{MHz}, \mathrm{CDCl}_{3}\right): \delta 7.48-7.42(\mathrm{~m}, 2 \mathrm{H}), 7.40-7.33(\mathrm{~m}, 3 \mathrm{H}), 7.10(\mathrm{td}, J=7.7,1.3 \mathrm{~Hz}, 1 \mathrm{H}), 6.94$ $(\mathrm{ddd}, J=7.4,1.3,0.5 \mathrm{~Hz}, 1 \mathrm{H}), 6.67$ (td, $J=7.4,1.0 \mathrm{~Hz}, 1 \mathrm{H}), 6.42$ (br.d, $J=7.9, \mathrm{~Hz}, 1 \mathrm{H}), 5.16$ $(\mathrm{d}, J=10.6 \mathrm{~Hz}, 1 \mathrm{H}), 5.00(\mathrm{~d}, J=10.6 \mathrm{~Hz}, 1 \mathrm{H}), 4.47(\mathrm{~s}, 1 \mathrm{H}), 3.38(\mathrm{qd}, J=7.2,1.5 \mathrm{~Hz}, 2 \mathrm{H}), 1.21$ $(\mathrm{s}, 3 \mathrm{H}), 1.19(\mathrm{~s}, 3 \mathrm{H}), 1.12(\mathrm{t}, J=7.1 \mathrm{~Hz}, 3 \mathrm{H}), 1.02(\mathrm{~s}, 3 \mathrm{H}) ;{ }^{13} \mathrm{C} \mathrm{NMR}\left(126 \mathrm{MHz}, \mathrm{CDCl}_{3}\right): \delta$ 174.4, 149.2, 135.2, 132.4, 129.8, 129.0, 128.4, 124.2, 120.9, 117.8, 107.2, 85.5, 77.1, 51.4, 43.7, 41.4, 24.5, 22.1, 21.8, 11.8; FT-IR (neat): 3031, 2972, 2932, 2872, 1702, 1604, 1486, 1454, 1393, 1375, 1350, 1313, 1264, $1216 \mathrm{~cm}^{-1}$; HRESI-MS: calculated for $\mathrm{C}_{22} \mathrm{H}_{26} \mathrm{~N}_{2} \mathrm{O}_{2} \mathrm{Na}(\mathrm{M}+\mathrm{Na})^{+}$ 373.1886, observed 373.1886 .

\section{( \pm )-(3a $R, 8 \mathrm{a} S)-1-P h e n y l m e t h o x y-3,3,3 a-t r i m e t h y l-8-(p r o p-2-e n-1-y l)-3,3 a, 8,8 a-$} tetrahydropyrrolo[2,3-b]indol-2(1H)-one (10n)<smiles>C=CCN1c2ccccc2C2(C)C1N(Cc1ccccc1)C(=O)C2(C)C</smiles>

Prepared in $83 \%$ yield $(88 \mathrm{mg}, 0.24 \mathrm{mmol})$ as a colorless oil from the reaction of 1-allyl-3methylindole (50 mg, $0.29 \mathrm{mmol}$ ) with 2-bromo-2-methyl- $N$-(phenylmethoxy)propanamide (95 mg, $35 \mathrm{mmol}$ ) via general procedure A. $R_{f}=0.34$ (8:2, hexanes: ethyl acetate); ${ }^{1} \mathrm{H}$ NMR (500 $\left.\mathrm{MHz}_{\mathrm{CDCl}}\right): \delta 7.48-7.43(\mathrm{~m}, 2 \mathrm{H}), 7.40-7.34(\mathrm{~m}, 3 \mathrm{H}), 7.09(\mathrm{td}, J=7.8,1.3 \mathrm{~Hz}, 1 \mathrm{H}), 6.95$ (ddd, $J=7.4,1.3,0.6 \mathrm{~Hz}, 1 \mathrm{H}), 6.70$ (td, $J=7.4,1.0 \mathrm{~Hz}, 1 \mathrm{H}), 6.45$ (br.d, $J=8.0 \mathrm{~Hz}, 1 \mathrm{H}), 5.82$ 
(dddd, $J=17.0,10.2,6.5,4.5 \mathrm{~Hz}, 1 \mathrm{H}), 5.22(\mathrm{dq}, J=17.1,1.7 \mathrm{~Hz}, 1 \mathrm{H}), 5.18(\mathrm{dq}, J=10.2,1.7$ $\mathrm{Hz}, 1 \mathrm{H}), 5.17$ (d, $J=10.4 \mathrm{~Hz}, 1 \mathrm{H}), 5.00$ (d, $J=10.3 \mathrm{~Hz}, 1 \mathrm{H}), 4.55$ (s, 1H), 4.00 (ddt, $J=16.5$, 4.5, $1.7 \mathrm{~Hz}, 1 \mathrm{H}), 3.89$ (ddt, $J=16.5,6.6,1.4 \mathrm{~Hz}, 1 \mathrm{H}), 1.22(\mathrm{~s}, 3 \mathrm{H}), 1.21$ (s, 3H), 1.02 (s, 3H); ${ }^{13} \mathrm{C}$ NMR (126 MHz, $\left.\mathrm{CDCl}_{3}\right): \delta 174.4,149.6,135.1,133.3,132.2,129.8,129.0,128.6,128.4$, 124.1, 118.3, 117.4, 107.7, 85.9, 77.1, 51.5, 50.2, 43.7, 24.6, 22.2, 21.6; FT-IR (neat): 3031, 2974, 2932, 1704, 1643, 1485, 1454, 1399, 1375, 1361, 1303, 1284, 1262, $1215 \mathrm{~cm}^{-1}$; HRESIMS: calculated for $\mathrm{C}_{23} \mathrm{H}_{26} \mathrm{~N}_{2} \mathrm{O}_{2} \mathrm{Na}(\mathrm{M}+\mathrm{Na})^{+} 385.1886$, observed 385.1890.

( \pm )-(3a $R, 8 \mathrm{a} S)$-1-Phenylmethoxy-3,3,3a-trimethyl-8-[(1,1-dimethylethyl)dimethylsilyl]3,3a,8,8a-tetrahydropyrrolo $[2,3-b]$ indol-2(1H)-one (10o)<smiles>CC1(C)C(=O)N(OCc2ccccc2)[C@H]2[C@H]1c1ccccc1N2Cc1ccccc1</smiles>

Prepared in $92 \%$ yield $(178 \mathrm{mg}, 0.41 \mathrm{mmol})$ as a white solid from the reaction of $1-(t$ butyldimethylsilyl)-3-methylindole $(0.1 \mathrm{~g}, \quad 0.41 \mathrm{mmol})$ with 2-bromo-2-methyl- $N$ (phenylmethoxy)propanamide $(132.5 \mathrm{mg}, 0.49 \mathrm{mmol})$ via general procedure A. $R_{f}=0.5(3: 1$, hexanes: ethyl acetate); mp $109.5-111.8{ }^{\circ} \mathrm{C} ;{ }^{1} \mathrm{H}$ NMR $\left(500 \mathrm{MHz}, \mathrm{CDCl}_{3}\right): \delta 7.43-7.40(\mathrm{~m}$, 2H), $7.35-7.29(\mathrm{~m}, 3 \mathrm{H}), 7.16(\mathrm{ddd}, J=7.6,1.4,0.5 \mathrm{~Hz}, 1 \mathrm{H}), 7.08$ (ddd, $J=8.0,7.4,1.3 \mathrm{~Hz}$, 1H), 6.89 (ddd, $J=8.0,1.0,0.5 \mathrm{~Hz}, 1 \mathrm{H}), 6.77$ (br.d, $J=7.5, \mathrm{~Hz}, 1 \mathrm{H}), 5.08$ (s, 1H), 5.04 (d, $J=$ $9.5 \mathrm{~Hz}, 1 \mathrm{H}), 4.86(\mathrm{~d}, J=9.6 \mathrm{~Hz}, 1 \mathrm{H}), 1.43(\mathrm{~s}, 3 \mathrm{H}), 1.26(\mathrm{~s}, 3 \mathrm{H}), 1.26(\mathrm{~s}, 3 \mathrm{H}), 0.90(\mathrm{~s}, 9 \mathrm{H}), 0.53$ $(\mathrm{s}, 3 \mathrm{H}), 0.38(\mathrm{~s}, 3 \mathrm{H}) ;{ }^{13} \mathrm{C} \mathrm{NMR}\left(101 \mathrm{MHz}, \mathrm{CDCl}_{3}\right): \delta 172.8,149.1,136.4,134.9,129.0,128.5$, $128.4,127.9,126.2,119.3,113.0,83.0,78.0,54.0,43.5,27.1,24.6,20.9,20.7,19.7,-2.6,-3.8$; FT-IR (neat): 3066, 3030, 2959, 1700, 1599, 1477, 1459, 1453, 1404, 1390, 1372, 1353, 1329, 1319, 1294, 1250, $1209 \mathrm{~cm}^{-1}$; HRESI-MS: calculated for $\mathrm{C}_{26} \mathrm{H}_{36} \mathrm{~N}_{2} \mathrm{O}_{2} \mathrm{SiNa}(\mathrm{M}+\mathrm{Na})^{+} \quad 459.2483$, observed 459.2454 . 
( \pm )-(3R,3a $S, 8 \mathrm{a} S)$-1-Phenylmethoxy-3-ethyl-3a,8-dimethyl-3,3a,8,8a-tetrahydropyrrolo[2,3b] indol-2(1H)-one and $( \pm)-(3 S, 3 a S, 8 a S)-1-p h e n y l m e t h o x y-3-e t h y l-3 a, 8-d i m e t h y l-3,3 a, 8,8 a-$ tetrahydropyrrolo[2,3-b]indol-2(1H)-one (12)<smiles>CCC1C(=O)N(Cc2ccccc2)[C@H]2N(C)c3ccccc3[C@]12C</smiles><smiles>CCC1C(=O)N(OCc2ccccc2)[C@H]2N(C)c3ccccc3[C@]12C</smiles>

Prepared in $51 \%$ yield $(59 \mathrm{mg}, 0.18 \mathrm{mmol})$ as a colorless oil from the reaction of 1,3dimethylindole (50 g, $0.35 \mathrm{mmol})$ with $( \pm)$-2-bromo- $N$-(phenylmethoxy)butanamide (112.5 mg, $0.41 \mathrm{mmol}$ ) via general procedure A. (Endo- and exo-diastereomers are inseparable and characterized as a mixture.) $R_{f}=0.64$ (7:3, hexanes: ethyl acetate); ${ }^{1} \mathrm{H}$ NMR $\left(500 \mathrm{MHz}, \mathrm{CDCl}_{3}\right)$ : $\delta 7.50-7.47(\mathrm{~m}, 4 \mathrm{H}), 7.43-7.37(\mathrm{~m}, 6 \mathrm{H}), 7.16(\mathrm{td}, J=7.7,1.3 \mathrm{~Hz}, 1 \mathrm{H}), 7.13(\mathrm{td}, J=7.7,1.3$ Hz, 1H), 7.03 (ddd, $J=7.4,1.3,0.5 \mathrm{~Hz}, 1 \mathrm{H}), 7.00$ (ddd, $J=7.4,1.3,0.5 \mathrm{~Hz}, 1 \mathrm{H}), 6.74-6.71$ (m, 2H), $6.47-6.42(\mathrm{~m}, 1 \mathrm{H}), 5.20(\mathrm{~d}, J=10.5 \mathrm{~Hz}, 1 \mathrm{H}), 5.13(\mathrm{~d}, J=10.7 \mathrm{~Hz}, 1 \mathrm{H}), 5.02(\mathrm{~d}, J=2.0$ Hz, 1H), 5.00 (d, J=1.8 Hz, 1H), 4.36 (s, 1H), 4.33 (s, 1H), 2.97 (s, 3H), 2.96 (s, 3H), 2.49 (dd, $J=7.5,6.6 \mathrm{~Hz}, 1 \mathrm{H}), 2.25(\mathrm{t}, J=6.9 \mathrm{~Hz}, 1 \mathrm{H}), 1.87-1.68(\mathrm{~m}, 2 \mathrm{H}), 1.60-1.53(\mathrm{~m}, 2 \mathrm{H}), 1.36(\mathrm{~s}$, $3 \mathrm{H}), 1.22(\mathrm{~s}, 3 \mathrm{H}), 1.16(\mathrm{t}, J=7.5 \mathrm{~Hz}, 3 \mathrm{H}), 1.03(\mathrm{t}, J=7.4 \mathrm{~Hz}, 3 \mathrm{H}) ;{ }^{13} \mathrm{C} \mathrm{NMR}(101 \mathrm{MHz}$, $\left.\mathrm{CDCl}_{3}\right): \delta 171.6,171.5,150.6,149.4,135.4,135.2,135.1,130.7,129.7,129.0,129.0,128.7$, 128.6, 128.6, 128.5, 124.4, 122.4, 118.5, 118.0, 107.2, 107.0, 88.5, 88.5, 77.1, 77.1, 50.9, 49.4, 48.2, 47.7, 34.5, 34.3, 27.5, 22.4, 20.7, 19.7, 12.8, 12.4; FT-IR (neat): 3054, 3031, 2962, 2933, 2875, 1704, 1605, 1489, 1454, 1428, 1371, 1326, 1301, 1269, 1239, $1216 \mathrm{~cm}^{-1}$; HRESI-MS: calculated for $\mathrm{C}_{21} \mathrm{H}_{25} \mathrm{~N}_{2} \mathrm{O}_{2}(\mathrm{M}+\mathrm{H})^{+} 337.1910$, observed 337.1917. 
( \pm )-(3aS,8aR)-1-Phenylmethoxy-3-(spirocyclohexane)-3a,8-dimethyl-3,3a,8,8atetrahydropyrrolo[2,3-b]indol-2(1H)-one (14)<smiles>CN1c2ccccc2[C@]2(C)CCCC[C@@H]2C(=O)N1OCc1ccccc1</smiles>

Prepared in $88 \%$ yield $(114 \mathrm{mg}, 0.30 \mathrm{mmol})$ as a colorless oil from the reaction of 1,3dimethylindole $(50 \mathrm{mg}, 0.34 \mathrm{mmol}$ ) with 2-bromo- $N$-(phenylmethoxy)cyclohexane carboxamide 13 (105 mg, $0.34 \mathrm{mmol}) \quad$ via general procedure A. $R_{f}=0.75$ (7:3, hexanes: ethyl acetate); ${ }^{1} \mathrm{H}$ NMR (500 MHz, $\left.\mathrm{CDCl}_{3}\right): \delta 7.49-7.47(\mathrm{~m}, 2 \mathrm{H}), 7.40-7.34(\mathrm{~m}, 3 \mathrm{H}), 7.14(\mathrm{td}, J=7.7,1.3 \mathrm{~Hz}$, $1 \mathrm{H}), 6.97$ (ddd, $J=7.4,1.3,0.5 \mathrm{~Hz}, 1 \mathrm{H}), 6.72$ (td, $J=7.4,1.0 \mathrm{~Hz}, 1 \mathrm{H}), 6.43$ (br.d, $J=7.9, \mathrm{~Hz}$, 1H), $5.16(\mathrm{~d}, J=10.5 \mathrm{~Hz}, 1 \mathrm{H}), 5.02(\mathrm{dd}, J=10.3,0.6 \mathrm{~Hz}, 1 \mathrm{H}), 4.31(\mathrm{~s}, 1 \mathrm{H}), 2.90$ (s, 3H), $2.16-$ $2.09(\mathrm{~m}, 1 \mathrm{H}), 1.86(\mathrm{dt}, J=13.3,4.4 \mathrm{~Hz}, 1 \mathrm{H}), 1.79-1.67(\mathrm{~m}, 2 \mathrm{H}), 1.65-1.55(\mathrm{~m}, 2 \mathrm{H}), 1.47$ $(\mathrm{ddd}, J=13.4,11.5,4.0 \mathrm{~Hz}, 1 \mathrm{H}), 1.34(\mathrm{~m}, 1 \mathrm{H}), 1.24(\mathrm{~s}, 3 \mathrm{H}), 1.21-1.12(\mathrm{~m}, 2 \mathrm{H}) ;{ }^{13} \mathrm{C} \mathrm{NMR}(101$ $\left.\mathrm{MHz}, \mathrm{CDCl}_{3}\right): \delta 174.8,151.1,135.2,131.6,129.7,128.9,128.6,128.5,124.3,118.2,107.3$, 88.9, 77.1, 52.7, 45.9, 34.9, 33.0, 30.1, 25.4, 22.1, 22.0, 21.9; FT-IR (neat): 3131, 2942, 2922, $2861,1898,1605,1485,1466,1452,1428,1410,1376,1365,1323,1293,1274,1212,1206 \mathrm{~cm}^{-}$

${ }^{1}$; HRESI-MS: calculated for $\mathrm{C}_{24} \mathrm{H}_{28} \mathrm{~N}_{2} \mathrm{O}_{2} \mathrm{Na}(\mathrm{M}+\mathrm{Na})^{+}$399.2043, observed 399.2039. 
( \pm )-(3R,3aS,8aR)-1-Phenylmethoxy-3-chloro-3a,8-dimethyl-3,3a,8,8atetrahydropyrrolo $[2,3-b]$ indol-2(1H)-one, $( \pm)-(3 S, 3 a S, 8 a R)-p h e n y l m e t h o x y-3-c h l o r o-3 a, 8-$ dimethyl-3,3a,8,8a-tetrahydropyrrolo[2,3-b]indol-2(1H)-one and $( \pm)-(3 R, 3 a R, 8 b S)-1-$ phenylmethoxy-3-chloro-4,8b-dimethyl-3,3a,4,8b-tetrahydropyrrolo $[3,2-b]$ indol-2(1H)-one (16)
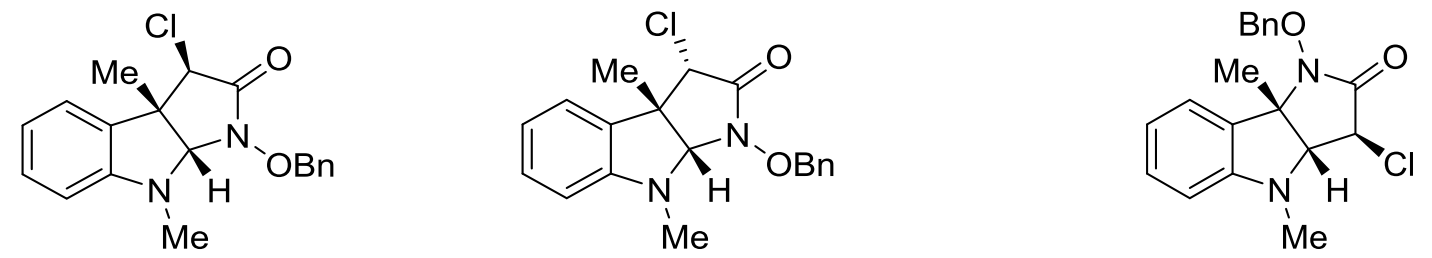

Exo:Endo:Minor regiosiomer

10:9:3 (from crude nmr analysis)

Prepared in $67 \%$ yield $(80 \mathrm{mg}, 0.23 \mathrm{mmol}$ ) from the reaction of 1,3-dimethyindole $(50 \mathrm{mg}, 0.34$ mmol) with 2,2-dichloro- $N$-(phenylmethoxy)acetamide $(81 \mathrm{mg}, 0.35 \mathrm{mmol}) \mathbf{1 5}$ via general procedure A. ( $2 \mathrm{~g}$ scale reaction gave diminished yield of $57 \%$ )

Exo: Yield $=30 \%$, pale yellow oil, $R_{f}=0.84$ (7:3, hexanes: ethyl acetate); ${ }^{1} \mathrm{H}$ NMR (500 MHz, $\left.\mathrm{CDCl}_{3}\right): \delta 7.52-7.49(\mathrm{~m}, 2 \mathrm{H}), 7.47-7.41(\mathrm{~m}, 3 \mathrm{H}), 7.18(\mathrm{td}, J=7.7,1.2 \mathrm{~Hz}, 1 \mathrm{H}), 7.03$ (dd, $J=$ $7.5,1.2 \mathrm{~Hz}, 1 \mathrm{H}), 6.76$ (td, $J=7.5,0.9 \mathrm{~Hz}, 1 \mathrm{H}), 6.44$ (br.d, $J=7.9 \mathrm{~Hz}, 1 \mathrm{H}), 5.15$ (d, $J=11.0 \mathrm{~Hz}$, $1 \mathrm{H}), 4.98(\mathrm{~d}, J=11.0 \mathrm{~Hz}, 1 \mathrm{H}), 4.55(\mathrm{~s}, 1 \mathrm{H}), 4.30(\mathrm{~s}, 1 \mathrm{H}), 2.98(\mathrm{~s}, 3 \mathrm{H}), 1.34(\mathrm{~s}, 3 \mathrm{H}) ;{ }^{13} \mathrm{C} \mathrm{NMR}$ $\left(126 \mathrm{MHz}, \mathrm{CDCl}_{3}\right): \delta 164.4,148.1,134.7,131.8,130.2,129.8,129.4,128.7,122.8,118.9,107.5$, 86.3, 76.8, 60.4, 50.0, 33.3, 21.1; FT-IR (neat): 3056, 3031, 2929, 2879, 1719, 1606, 1489, 1451, 1430, 1387, 1373, 1325, 1305, 1276, 1265, 1231, $1214 \mathrm{~cm}^{-1}$; HRESI-MS: calculated for $\mathrm{C}_{19} \mathrm{H}_{19} \mathrm{~N}_{2} \mathrm{O}_{2} \mathrm{ClNa}(\mathrm{M}+\mathrm{Na})^{+}$365.1027, observed 365.1026.

Endo:Yield $=28 \%$, white solid, $\mathrm{mp} 160.5-162.8^{\circ} \mathrm{C} ; R_{f}=0.37$ (7:3, hexanes: ethyl acetate); ${ }^{1} \mathrm{H}$ NMR (500 MHz, $\left.\mathrm{CDCl}_{3}\right): \delta 7.47-7.43(\mathrm{~m}, 2 \mathrm{H}), 7.41-7.38(\mathrm{~m}, 3 \mathrm{H}), 7.30-7.23(\mathrm{~m}, 1 \mathrm{H}), 7.18$ (ddd, $J=7.5,1.3 \mathrm{~Hz}, 1 \mathrm{H}), 6.76(\mathrm{td}, J=7.5,1.0 \mathrm{~Hz}, 1 \mathrm{H}), 6.45(\mathrm{br}, \mathrm{d}, J=7.9, \mathrm{~Hz}, 1 \mathrm{H}), 5.23$ (d, $J=$ $10.5 \mathrm{~Hz}, 1 \mathrm{H}), 4.96(\mathrm{~d}, J=10.5 \mathrm{~Hz}, 1 \mathrm{H}), 4.44(\mathrm{~s}, 1 \mathrm{H}), 4.41(\mathrm{~s}, 1 \mathrm{H}), 2.98(\mathrm{~s}, 3 \mathrm{H}), 1.43(\mathrm{~s}, 3 \mathrm{H}) ;{ }^{13} \mathrm{C}$ NMR (101 MHz, $\left.\mathrm{CDCl}_{3}\right): \delta$ 164.1, 149.7, 134.6, 129.8, 129.5, 129.4, 129.2, 128.7, 126.3, 118.4 , 107.3, 87.1, 77.3, 61.1, 50.4, 34.0, 25.5; FT-IR (neat): 3055, 3031, 2925, 2870, 2833, 1721, 1606, 1489, 1472, 1452, 1430, 1386, 1374, 1326, 1307, 1276, 1236, $1213 \mathrm{~cm}^{-1}$; HRESI-MS: calculated for $\mathrm{C}_{19} \mathrm{H}_{19} \mathrm{~N}_{2} \mathrm{O}_{2} \mathrm{ClNa}(\mathrm{M}+\mathrm{Na})^{+} 365.1027$, observed 365.1030.

Minor regioisomer: Yield $=9 \%$, colorless oil, $R_{f}=0.53$ (7:3, hexanes: ethyl acetate); ${ }^{1} \mathrm{H}$ NMR (500 MHz, CDCl3): $\delta 7.39$ - 7.34 (m, 5H), 7.31 (ddd, $J=7.4,1.4,0.6 \mathrm{~Hz}, 1 \mathrm{H}), 7.28$ (td, $J=7.7$, 
$1.3 \mathrm{~Hz}, 1 \mathrm{H}), 6.83$ (td, $J=7.4,0.9 \mathrm{~Hz}, 1 \mathrm{H}), 6.58$ (br.d, J = 7.9, $0.7 \mathrm{~Hz}, 1 \mathrm{H}), 5.12$ (d, $J=9.3 \mathrm{~Hz}$, 1H), 4.37 (d, $J=0.5 \mathrm{~Hz}, 1 \mathrm{H}), 4.35$ (d, $J=9.3 \mathrm{~Hz}, 1 \mathrm{H}), 3.78$ (d, $J=0.4 \mathrm{~Hz}, 1 \mathrm{H}), 2.89$ (s, 3H), 1.77 (s, 3H); ${ }^{13} \mathrm{C}$ NMR (101 MHz, CDCl3): $\delta 162.9,151.0,134.2,130.7,129.6,128.9,128.4$, 127.2, 124.1, 118.9, 108.0, 78.3, 77.2, 68.8, 52.7, 33.7, 21.3; FT-IR (neat): 3057, 3032, 2976, 2879, 2819, 1713, 1609, 1487, 1471, 1453, 1430, 1370, 1317, 1297, 1281, 1238, $1208 \mathrm{~cm}^{-1}$; HRESI-MS: calculated for $\mathrm{C}_{19} \mathrm{H}_{19} \mathrm{~N}_{2} \mathrm{O}_{2} \mathrm{ClNa}(\mathrm{M}+\mathrm{Na})^{+}$365.1027, observed 365.1024.

( \pm )-(3aS,8aR)-1-benzyloxy-3-chloro-3a,8-bis(3-methylbut-2-en-1-yl)-3,3a,8,8atetrahydropyrrolo $[2,3-b]$ indol-2(1H)-one (18)<smiles>CC(C)=CCN1c2ccccc2C2(CC=C(C)C)C1N(OCc1ccccc1)C(=O)[C@H]2Cl</smiles>

Prepared in $18 \%$ yield $(32 \mathrm{mg}, 0.07 \mathrm{mmol})$ as a colorless oil from the reaction of 1,3-bis(3methylbut-2-en-1-yl)-1H-indole $17(0.1 \quad$ g, $0.39 \quad \mathrm{mmol})$ with 2,2-dichloro- $N$ (phenylmethoxy)acetamide $(0.11 \mathrm{~g}, 0.47 \mathrm{mmol})$ via general procedure A. $R_{f}=0.25(9: 1$, hexanes: ethyl acetate); ${ }^{1} \mathrm{H}$ NMR $\left(500 \mathrm{MHz}, \mathrm{CDCl}_{3}\right): \delta 7.44-7.42(\mathrm{~m}, 2 \mathrm{H}), 7.39-7.38(\mathrm{~m}, 3 \mathrm{H})$, $7.36-7.31(\mathrm{~m}, 1 \mathrm{H}), 7.15(\mathrm{td}, J=7.7,1.3 \mathrm{~Hz}, 1 \mathrm{H}), 6.75(\mathrm{td}, J=7.5,1.0 \mathrm{~Hz}, 1 \mathrm{H}), 6.49(\mathrm{~d}, J=7.8$ $\mathrm{Hz}, 1 \mathrm{H}), 5.24$ (d, $J=10.4 \mathrm{~Hz}, 1 \mathrm{H}), 5.19-5.11(\mathrm{~m}, 1 \mathrm{H}), 4.96(\mathrm{~d}, J=10.4 \mathrm{~Hz}, 1 \mathrm{H}), 4.92-4.82$ $(\mathrm{m}, 1 \mathrm{H}), 4.72(\mathrm{~s}, 1 \mathrm{H}), 4.61(\mathrm{~s}, 1 \mathrm{H}), 3.90(\mathrm{~d}, J=6.5 \mathrm{~Hz}, 1 \mathrm{H}), 2.46(\mathrm{dd}, J=14.8,8.8 \mathrm{~Hz}, 1 \mathrm{H}), 2.26$ $(\mathrm{dd}, J=14.6,6.3 \mathrm{~Hz}, 1 \mathrm{H}), 1.70(\mathrm{~s}, 6 \mathrm{H}), 1.68(\mathrm{~s}, 3 \mathrm{H}), 1.59(\mathrm{~s}, 3 \mathrm{H}) ;{ }^{13} \mathrm{C} \mathrm{NMR}\left(101 \mathrm{MHz}, \mathrm{CDCl}_{3}\right)$ : $\delta 163.8,149.0,137.8,135.7,134.7,129.6,129.2$, 129.0, 128.6, 127.1, 120.0, 118.2 , 117.2, 110.0, 108.3, 81.8, 77.4, 58.3, 54.8, 45.0, 35.9, 26.0, 25.7, 18.1, 18.1; FT-IR (neat): 3033, 2965, 2918, 2853, 1729, 1604, 1487, 1463, 1454, 1377, 1320, 1261, $1211 \mathrm{~cm}^{-1}$; HRESI-MS: calculated for $\mathrm{C}_{27} \mathrm{H}_{31} \mathrm{~N}_{2} \mathrm{O}_{2} \mathrm{ClNa}(\mathrm{M}+\mathrm{Na})^{+} 473.1966$, observed 473.1975. 
( \pm )-(3aS,8a $R)-3 a, 8-D i m e t h y l-3,3 a, 8,8 a-t e t r a h y d r o p y r r o l o[2,3-b]$ indol-2(1H)-one (19)<smiles>CN1c2ccccc2[C@@]2(C)CC(=O)N[C@H]12</smiles>

To a solution of $16(0.2 \mathrm{~g}, 0.58 \mathrm{mmol})$ in dry THF $(0.25 \mathrm{M})$ was added dry methanol $(1.0 \mathrm{ml})$ and $40 \mathrm{ml}$ of $\mathrm{SmI}_{2}$ solution in THF $(0.1 \mathrm{M})$. The mixture was stirred under nitrogen for $6 \mathrm{~h}$ at room temperature and quenched with saturated ammonium chloride solution. The mixture was extracted with ethyl acetate, washed with saturated $\mathrm{Na}_{2} \mathrm{~S}_{2} \mathrm{O}_{3}$ solution, dried over anhydrous sodium sulfate, the solvent was removed under reduced pressure and purified by column chromatography (100\% ethyl acetate to $10 \%$ methanol in ethyl aceteate) to afford $\mathbf{1 9}$ a white solid in 97\% yield (113 mg, $0.56 \mathrm{mmol})$.

Alternatively,<smiles>CN1c2ccccc2C2(C)C1N(Cc1ccccc1)C(=O)[C@H]2Cl</smiles>

16

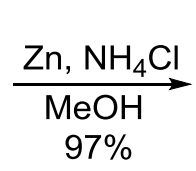

$97 \%$

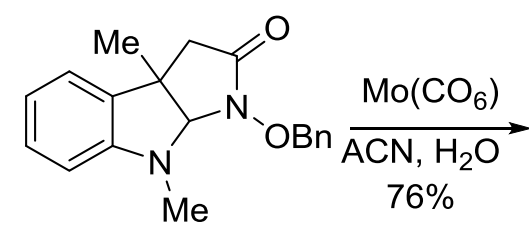

23

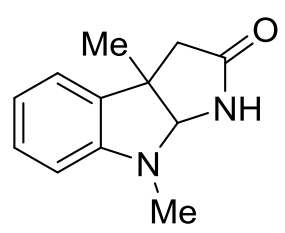

20

( \pm )-(3aS,8a $R)$-1-phenylmethoxy-3a,8-dimethyl-3,3a,8,8a-tetrahydropyrrolo[2,3-b]indol2(1H)-one (23)<smiles>CN1c2ccccc2C2(C)CC(=O)N(OCc3ccccc3)C12</smiles>

To a solution of 16 ( $50 \mathrm{mg}, 0.15 \mathrm{mmol})$ in methanol $(0.25 \mathrm{M})$ added ammonium chloride ( 47 $\mathrm{mg}, 0.9 \mathrm{mmol})$ and then zinc dust $(96 \mathrm{mg}, 1.46 \mathrm{mmol})$ protionwise over $10 \mathrm{~min}$ and heated for $30 \mathrm{~min}$ at $50{ }^{\circ} \mathrm{C}$ ). The mixture filtered on a Celite pad, concentrated under reduced pressure, and purified by column chromatography (1:1, ethyl acetate: hexane) to afford 22 as colorless oil in 95\% yield (40.8 mg, $0.14 \mathrm{mmol}) . R_{f}=0.58$ (1:9, hexanes: ethyl acetate); ${ }^{1} \mathrm{H}$ NMR (500 MHz, $\left.\mathrm{CDCl}_{3}\right): \delta 7.47-7.44(\mathrm{~m}, 2 \mathrm{H}), 7.42-7.38(\mathrm{~m}, 3 \mathrm{H}), 7.13(\mathrm{td}, J=7.7,1.2 \mathrm{~Hz}, 1 \mathrm{H}), 7.00(\mathrm{dt}, J=$ 7.3, $0.6 \mathrm{~Hz}, 1 \mathrm{H}), 6.73(\mathrm{td}, J=7.4,1.0 \mathrm{~Hz}, 1 \mathrm{H}), 6.42$ (d, $J=8.0 \mathrm{~Hz}, 1 \mathrm{H}), 5.18$ (d, $J=10.5 \mathrm{~Hz}$, 
1H), $4.95(\mathrm{~d}, J=10.5 \mathrm{~Hz}, 1 \mathrm{H}), 4.44(\mathrm{~s}, 1 \mathrm{H}), 2.98(\mathrm{~s}, 3 \mathrm{H}), 2.72(\mathrm{~d}, J=17.0 \mathrm{~Hz}, 1 \mathrm{H}), 2.50(\mathrm{~d}, J=$ $17.1 \mathrm{~Hz}, 1 \mathrm{H}), 1.33(\mathrm{~s}, 3 \mathrm{H}) ;{ }^{13} \mathrm{C} \mathrm{NMR}\left(126 \mathrm{MHz}, \mathrm{CDCl}_{3}\right): \delta 168.9,148.9,135.1,134.8,129.7$, 129.0, 128.8, 128.6, 122.2, 118.5, 107.0, 88.3, 77.1, 43.8, 41.6, 33.7, 26.2; FT-IR (neat): 3054, 3031, 2957, 2924, 2869, 1702, 1642, 1607, 1491, 1452, 1430, 1411, 1374, 1327, 1278, 1231, $1202 \mathrm{~cm}^{-1}$; HRESI-MS: calculated for $\mathrm{C}_{19} \mathrm{H}_{20} \mathrm{~N}_{2} \mathrm{O}_{2} \mathrm{Na}(\mathrm{M}+\mathrm{Na})^{+} 331.1417$, observed 331.1419.

To a solution of $( \pm)-(3 \mathrm{a} S, 8 \mathrm{a} R)-1$-phenylmethoxy-3a,8-dimethyl-3,3a,8,8a-tetrahydropyrrolo[2,3$b$ ]indol-2(1H)-one 23 in $\mathrm{CH}_{3} \mathrm{CN} / \mathrm{H}_{2} \mathrm{O}$ (9:1, degassed under nitrogen) was added $\mathrm{Mo}(\mathrm{CO})_{6}(1$ equiv.) and again degassed under nitrogen. The reaction mixture was vigorously stirred and heated to reflux for 12 hours under nitrogen. The consumption of starting material was confirmed by TLC. The crude mixture was passed through Celite bed and the Celite was washed with ethyl acetate. The collected filtrate was concentrated under reduced pressure. The crude product was purified by flash column chromatography ( $10 \%$ methanol : ethyl acetate) to afford, 19 a white solid in $76 \%$ yield ( $89 \mathrm{mg}, 0.44 \mathrm{mmol}) . R_{f}=0.55$ (1:9, methanol: ethyl acetate); $\mathrm{mp}$ $178.1-180.7{ }^{\circ} \mathrm{C} ;{ }^{1} \mathrm{H}$ NMR $\left(500 \mathrm{MHz}, \mathrm{CDCl}_{3}\right): \delta 7.31(\mathrm{~s}, 1 \mathrm{H}), 7.14(\mathrm{td}, J=7.7,1.3 \mathrm{~Hz}, 1 \mathrm{H})$, 7.05 (ddd, $J=7.3,1.3,0.6 \mathrm{~Hz}, 1 \mathrm{H}), 6.74$ (td, $J=7.4,1.0 \mathrm{~Hz}, 1 \mathrm{H}$ ), 6.43 (br.d, $J=7.8, \mathrm{~Hz}, 1 \mathrm{H}$ ), $4.84(\mathrm{~s}, 1 \mathrm{H}), 2.86(\mathrm{~s}, 3 \mathrm{H}), 2.72(\mathrm{~d}, J=17.1 \mathrm{~Hz}, 1 \mathrm{H}), 2.53(\mathrm{~d}, J=17.1 \mathrm{~Hz}, 1 \mathrm{H}), 1.47(\mathrm{~s}, 3 \mathrm{H}) ;{ }^{13} \mathrm{C}$ NMR (101 MHz, $\left.\mathrm{CDCl}_{3}\right): \delta 177.9,149.1,135.1,128.6,122.5,118.4,107.0,85.8,47.9,44.4$, 31.7, 25.1; FT-IR (neat): 3433, 3164, 3081, 2966, 2929, 2878, 2828, 1683, 1604, 1487, 1446, 1425, 1412, 1365, 1297, 1278, $1222 \mathrm{~cm}^{-1}$; HRESI-MS: $\mathrm{C}_{12} \mathrm{H}_{14} \mathrm{~N}_{2} \mathrm{ONa}(\mathrm{M}+\mathrm{Na})^{+} 225.0998$, observed 225.1002 .

( \pm )-(3aS,8aS)-1,3a,8-trimethyl-3,3a,8,8a-tetrahydropyrrolo[2,3-b]indol-2(1H)-one (20)

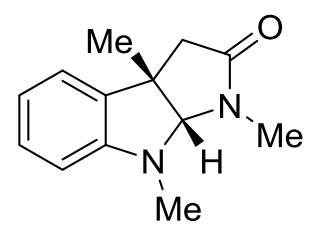

To a stirred solution $\mathrm{NaH}$ (60\% dispersion in mineral oil, $42 \mathrm{mg}, 1.04 \mathrm{mmol})$ in dry DMF $(0.25$ M) at $0{ }^{\circ} \mathrm{C}$ under nitrogen, was added $19(105 \mathrm{mg}, 0.52)$ and the mixture was stirred for $30 \mathrm{~min}$ at room temperature. The reaction mixture was cooled at $0{ }^{\circ} \mathrm{C}$ and methyl iodide $(39 \mu 1,0.62$ 
mmol) was added dropwise and stirred for $3 \mathrm{~h}$ at room temperature. The reaction mixture was quenched with saturated ammonium chloride $(5 \mathrm{ml})$, extracted with diethylether $(3 \mathrm{x} 10 \mathrm{ml})$. The phases were separated and the organic layer was washed with brine and dried over anhydrous sodium sulfate. The volatiles were removed under reduced pressure and purified by column chromatography (100\% ethyl acetate to $10 \%$ methanol in ethyl acetate) to get the title compound 20 as a white solid in $85 \%$ yield $(95.6 \mathrm{mg}, 0.44 \mathrm{mmol}) . R_{f}=0.6(1: 9$, methanol: ethyl acetate); $\operatorname{mp} 70.5-72.6{ }^{\circ} \mathrm{C} ;{ }^{1} \mathrm{H}$ NMR $\left(500 \mathrm{MHz}, \mathrm{CDCl}_{3}\right): \delta 7.14(\mathrm{td}, J=7.7,1.3 \mathrm{~Hz}, 1 \mathrm{H}), 7.04(\mathrm{ddd}, J=$ 7.3, 1.3, 0.5 Hz, 1H), 6.75 (td, $J=7.4,1.0 \mathrm{~Hz}, 1 \mathrm{H}), 6.47$ (br.d, $J=7.9, \mathrm{~Hz}, 1 \mathrm{H}), 4.62$ (s, 1H), $3.07(\mathrm{~s}, 3 \mathrm{H}), 2.96$ (t, $J=0.6 \mathrm{~Hz}, \mathrm{H}), 2.77$ (dq, $J=17.0,0.7 \mathrm{~Hz}, 1 \mathrm{H}), 2.55$ (dq, $J=17.1,0.8 \mathrm{~Hz}$, $1 \mathrm{H}), 1.45(\mathrm{~s}, 3 \mathrm{H}) ;{ }^{13} \mathrm{C}$ NMR $\left(126 \mathrm{MHz}, \mathrm{CDCl}_{3}\right): \delta 173.2,149.2,135.4,128.5,122.5,118.7$, 107.4, 92.3, 45.7, 44.2, 35.6, 28.6, 26.7; FT-IR (neat): 3053, 3020, 2955, 2921, 2861, 2826, 2793, 1674, 1606, 1489, 1472, 1452, 1436, 1425, 1415, 1395, 1333, 1298, 1281, 1251, 1231, $1204 \mathrm{~cm}^{-1}$; HRESI-MS: calculated for $\mathrm{C}_{13} \mathrm{H}_{16} \mathrm{~N}_{2} \mathrm{ONa}(\mathrm{M}+\mathrm{Na})^{+}$239.1155, observed 239.1163.

( \pm )-(3aS,8a $R)-1,3 a, 8-t r i m e t h y l-1,2,3,3 a, 8,8 a-h e x a h y d r o p y r r o l o[2,3-b]$ indole (21)

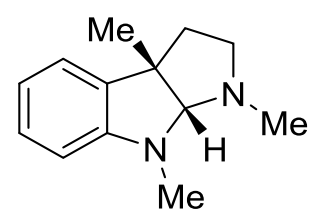

To a stirred solution of $\mathrm{LiAlH}_{4}(1.85 \mathrm{ml}, 1.84 \mathrm{mmol})$ in THF (1.0 M) was added 20 ( $47 \mathrm{mg}, 0.22$ $\mathrm{mmol})$ in THF $(3.5 \mathrm{ml})$ and the mixture was refluxed for $2 \mathrm{~h}$. The mixture was then cooled to 0 ${ }^{\circ} \mathrm{C}$ and quenched by adding ethyl acetate $(20 \mathrm{ml})$ dropwise. The organic phase was separated and washed with brine, dried over anhydrous sodium sulfate. The solvent was evaporated in vacuo and crude product was purified by column chromatography to get 21 as a colorless oil in $95 \%$ yield (42 mg, $0.21 \mathrm{mmol}) . R_{f}=0.65$ (1:9, methanol: ethyl acetate); ${ }^{1} \mathrm{H}$ NMR (500 $\left.\mathrm{MHz}, \mathrm{CDCl}_{3}\right)$ : $\delta 7.08(\mathrm{td}, J=7.7,1.3 \mathrm{~Hz}, 1 \mathrm{H}), 6.99(\mathrm{ddd}, J=7.2,1.3,0.5 \mathrm{~Hz}, 1 \mathrm{H}), 6.67(\mathrm{td}, J=7.4,1.0 \mathrm{~Hz}$, 1H), 6.41 (br.d, $J=7.8, \mathrm{~Hz}, 1 \mathrm{H}), 4.12(\mathrm{~s}, 1 \mathrm{H}), 2.95$ (s, 3H), 2.73 (ddd, $J=9.3,6.0,4.7 \mathrm{~Hz}, 1 \mathrm{H})$, $2.64(\mathrm{ddd}, J=9.3,7.9,6.9 \mathrm{~Hz}, 1 \mathrm{H}), 2.56(\mathrm{~s}, 3 \mathrm{H}), 1.99-1.94(\mathrm{~m}, 2 \mathrm{H}), 1.44(\mathrm{~s}, 3 \mathrm{H}) ;{ }^{13} \mathrm{C}$ NMR $\left(101 \mathrm{MHz} \mathrm{CDCl}_{3}\right): \delta 151.9,136.7,127.7,122.2,117.5,106.6,97.5,53.3,52.7,40.8,38.5,36.6$, 27.4; FT-IR (neat): 3049, 2955, 2926, 2852, 2789, 1605, 1489, 1449, 1425, 1373, 1345, 1331, 1298, $1254 \mathrm{~cm}^{-1}$; HRESI-MS: calculated for $\mathrm{C}_{13} \mathrm{H}_{18} \mathrm{~N}_{2} \mathrm{Na}(\mathrm{M}+\mathrm{Na})^{+}$225.1362, observed 
( \pm )-(3aS,8aR)-3a,8-bis(3-methylbut-2-en-1-yl)-3,3a,8,8a-tetrahydropyrrolo[2,3-b]indol2(1H)-one (24) ${ }^{12}$

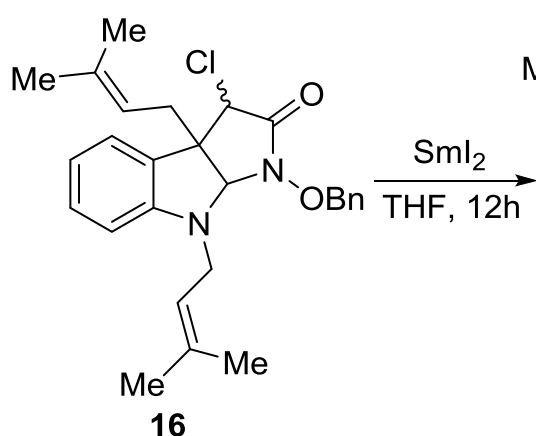

16

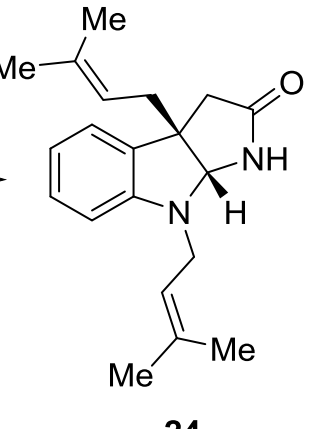

24

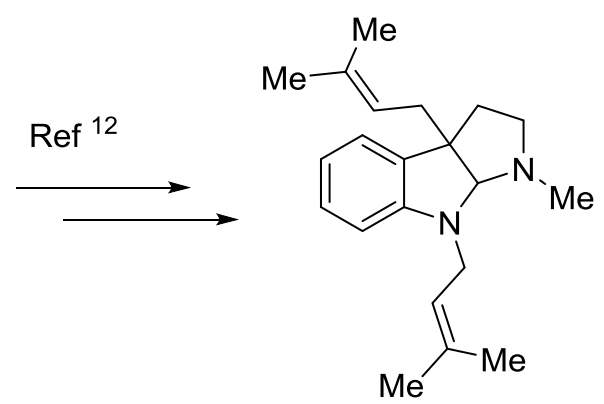

25 ( \pm )-Debromoflustramine B

To a solution of $18(20 \mathrm{mg}, 44.4 \mu \mathrm{mol})$ in dry THF $(0.25 \mathrm{M})$ was added dry methanol $(0.1 \mathrm{ml})$ and a $0.1 \mathrm{M}$ solution of $\mathrm{SmI}_{2}$ in THF $(3.1 \mathrm{ml})$. The mixture was stirred under nitrogen for $6 \mathrm{~h}$ at room temperature and quenched with saturated ammonium chloride solution. The mixture was extracted with ethyl acetate, washed with the saturated $\mathrm{Na}_{2} \mathrm{~S}_{2} \mathrm{O}_{3}$ solution, dried over anhydrous sodium sulfate, the solvent was removed under reduced pressure and purified by column chromatography (100\% ethyl acetate to $10 \%$ methanol in ethyl acetate) to afford 24 , a colorless oil, in $82 \%$ yield $(11.2 \mathrm{mg}, 36.4 \mu \mathrm{mol}) . R_{f}=0.70$ (19:1, ethyl acetate : methanol); ${ }^{1} \mathrm{H}$ NMR (500 $\mathrm{MHz}_{\mathrm{CDCl}}$ ): $\delta 7.13(\mathrm{t}, J=7.6 \mathrm{~Hz}, 1 \mathrm{H}), 7.04(\mathrm{~d}, J=7.3 \mathrm{~Hz}, 1 \mathrm{H}), 6.73(\mathrm{t}, J=7.4 \mathrm{~Hz}, 1 \mathrm{H}), 6.47$ (d, $J=7.9 \mathrm{~Hz}, 1 \mathrm{H}), 5.94$ (br.s, 1H), 5.31 (br.t, $J=6.6 \mathrm{~Hz}, 1 \mathrm{H}), 5.09$ ( br.t, $J=7.4 \mathrm{~Hz}, 1 \mathrm{H}$ ), 4.92 (s, 1H), $3.86(\mathrm{dd}, J=15.0,6.6 \mathrm{~Hz}, 1 \mathrm{H}), 3.77(\mathrm{dd}, J=14.9,6.8 \mathrm{~Hz}, 1 \mathrm{H}), 2.65(\mathrm{~d}, J=17.0 \mathrm{~Hz}$, 1H), 2.60 (d, $J=17.0 \mathrm{~Hz}, 1 \mathrm{H}), 2.40$ (qd, $J=14.3,6.5 \mathrm{~Hz}, 2 \mathrm{H}), 1.76$ (s, 3H), 1.74 (s, 3H), 1.71 (s, $3 \mathrm{H}), 1.58(\mathrm{~s}, 3 \mathrm{H}) ;{ }^{13} \mathrm{C} \mathrm{NMR}\left(126 \mathrm{MHz}, \mathrm{CDCl}_{3}\right): \delta 176.5,148.7,136.5,135.8,134.4,128.7$, 123.3, 119.9, 118.6, 118.5, 107.4, 81.0, 52.3, 43.6, 41.2, 36.4, 26.0, 25.8, 18.1, 18.0; FT-IR (neat): 3197, 3050, 2966, 2915, 2854, 1693, 1604, 1485, 1461, 1375, 1288, 1258, $1207 \mathrm{~cm}^{-1}$; HRESI-MS: calculated for $\mathrm{C}_{20} \mathrm{H}_{27} \mathrm{~N}_{2} \mathrm{O}(\mathrm{M}+\mathrm{H})^{+} 311.2118$, observed 311.2120. 


\section{References:}

(1) Xiong, H.; Xu, H.; Liao, S.; Xie, Z.; Tang, Y. J. Am. Chem. Soc. 2013, 135, 7851.

(2) (a) Su, Y. M.; Hou, Y.; Yin, F.; Xu, Y.-M.; Li, Y.; Zheng, X.; Wang, X. S. Org. Lett. 2014, 16, 2958.

(b) Nadres, E. T.; Lazareva, A.; Daugulis, O. J. Org. Chem. 2011, 76, 471.

(3) Dhanak, D.; Reese, C. B. J. Chem. Soc.-Perk. Trans. 1 1986, 12, 2181.

(4) Pitre, S. P.; McTiernan, C. D.; Ismaili, H.; Scaiano, J. C. Acs Catalysis 2014, 4, 2530.

(5) Roy, S.; Roy, S.; Gribble, G. W. Org. Lett. 2006, 8, 4975.

(6) Pierce, L. T.; Cahill, M. M.; McCarthy, F. O. Tetrahedron 2011, 67, 4601.

(7) Porcheddu, A.; Mura, M. G.; De Luca, L.; Pizzetti, M.; Taddei, M. Org. Lett. 2012, 14, 6112.

(8) Abel, E.; Maguire, G. E. M.; Murillo, O.; Suzuki, I.; De Wall, S. L.; Gokel, G. W. J. Am. Chem. Soc. 1999, 121, 9043.

(9) Wu, H.; Xue, F.; Xiao, X.; Qin, Y. J. Am. Chem. Soc. 2010, 132, 14052.

(10) Yadav, J. S.; Reddy, B. V. S.; Reddy, P. M.; Srinivas, C. Tet. Lett. 2002, 43, 5185.

(11) Jeffrey, C. S.; Barnes, K. L.; Eickhoff, J. A.; Carson, C. R. J. Am. Chem. Soc. 2011, 133, 7688.

(12) Cardoso, A. S. P.; Marques, M. M. B.; Srinivasan, N.; Prabhakar, S.; Lobo, A. M., Tetrahedron 2007, 63 , 10211. 


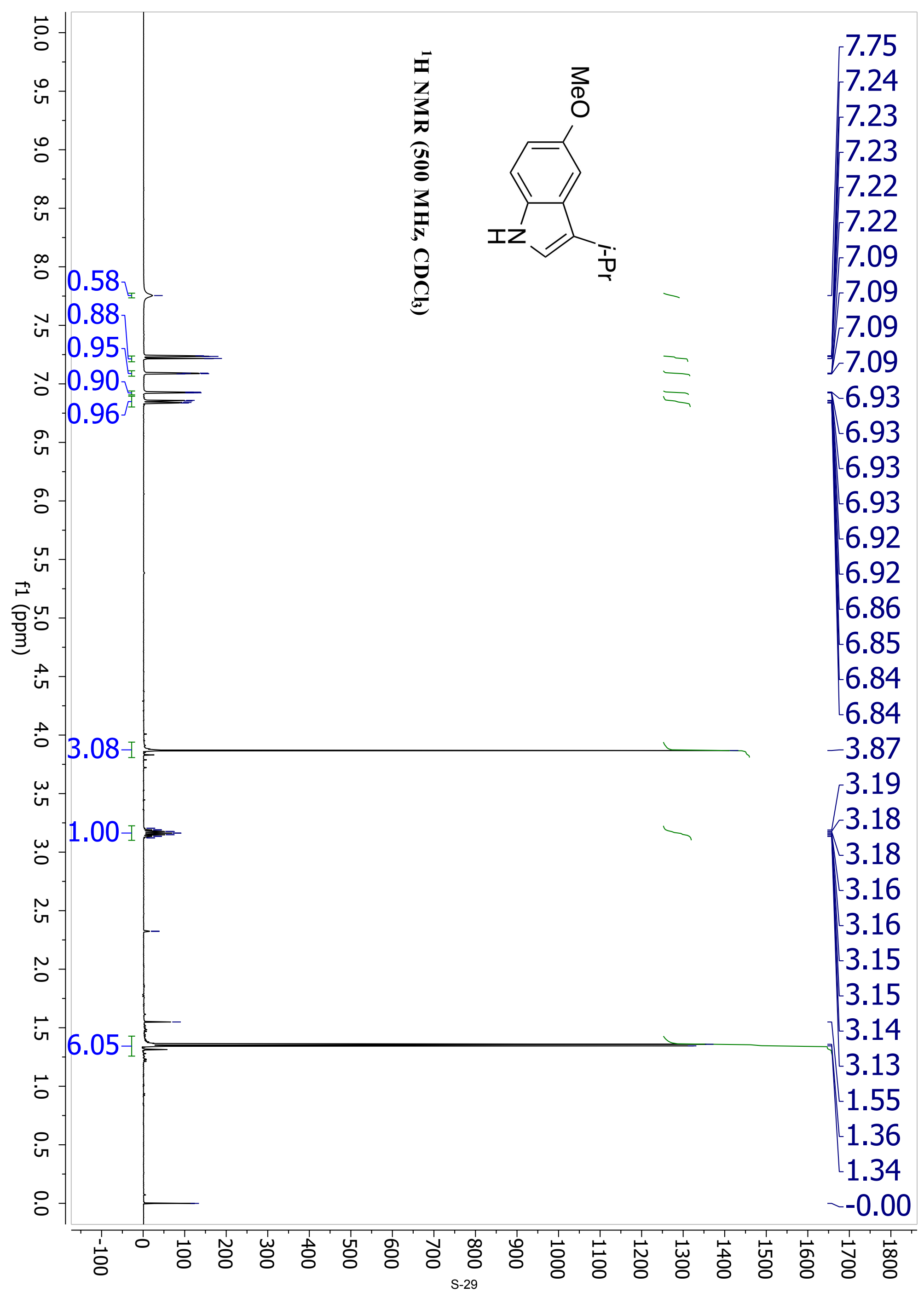




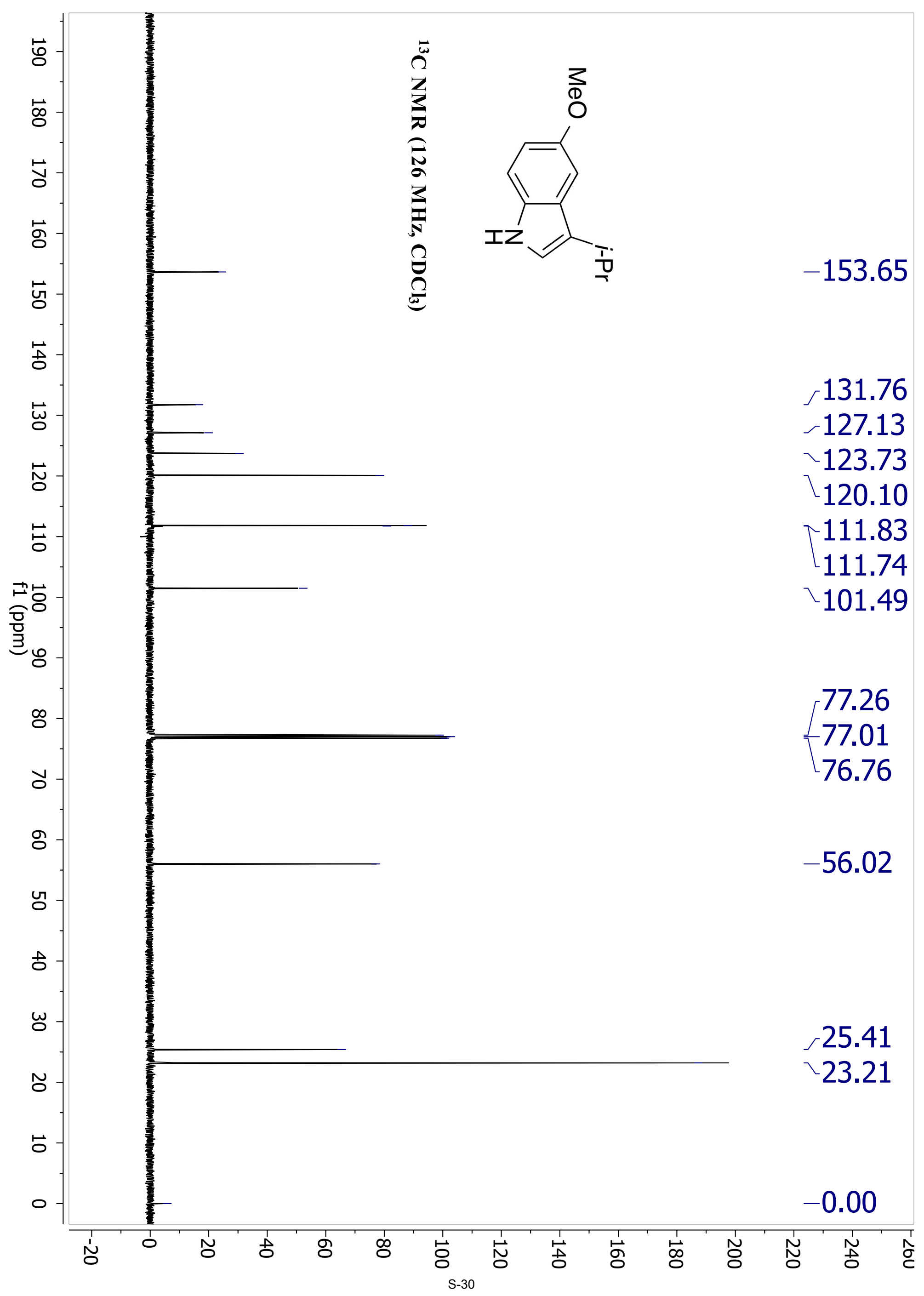




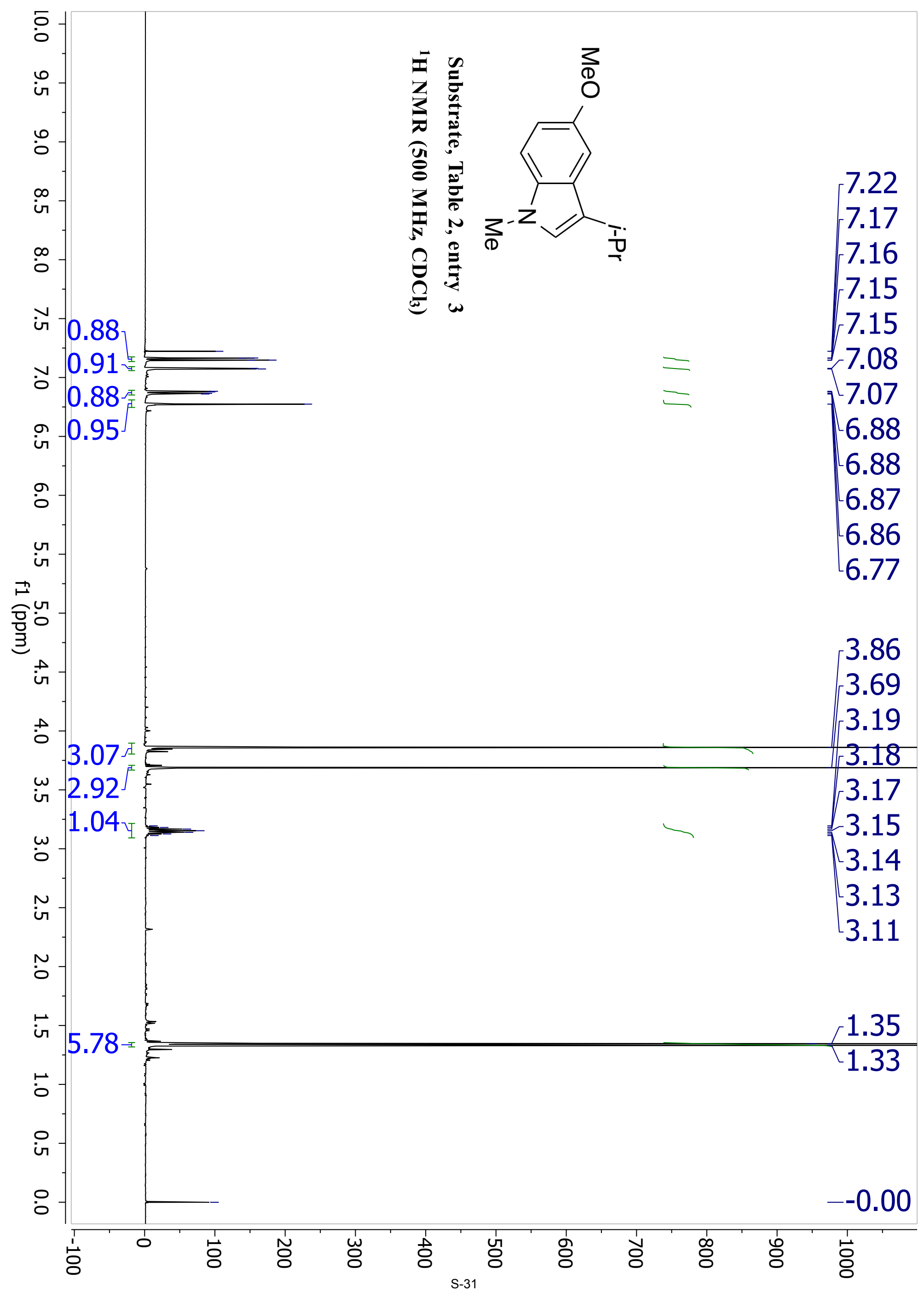




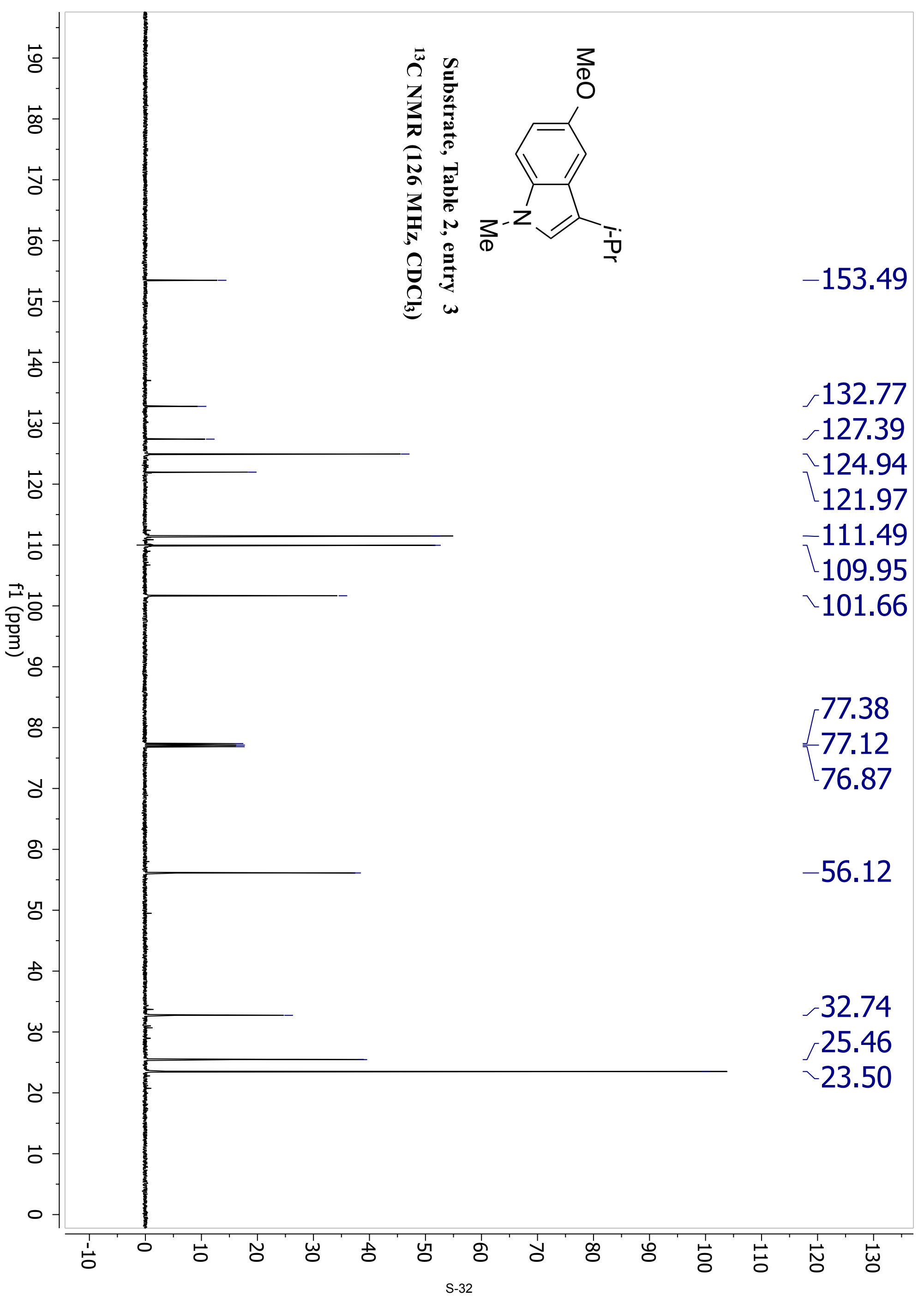




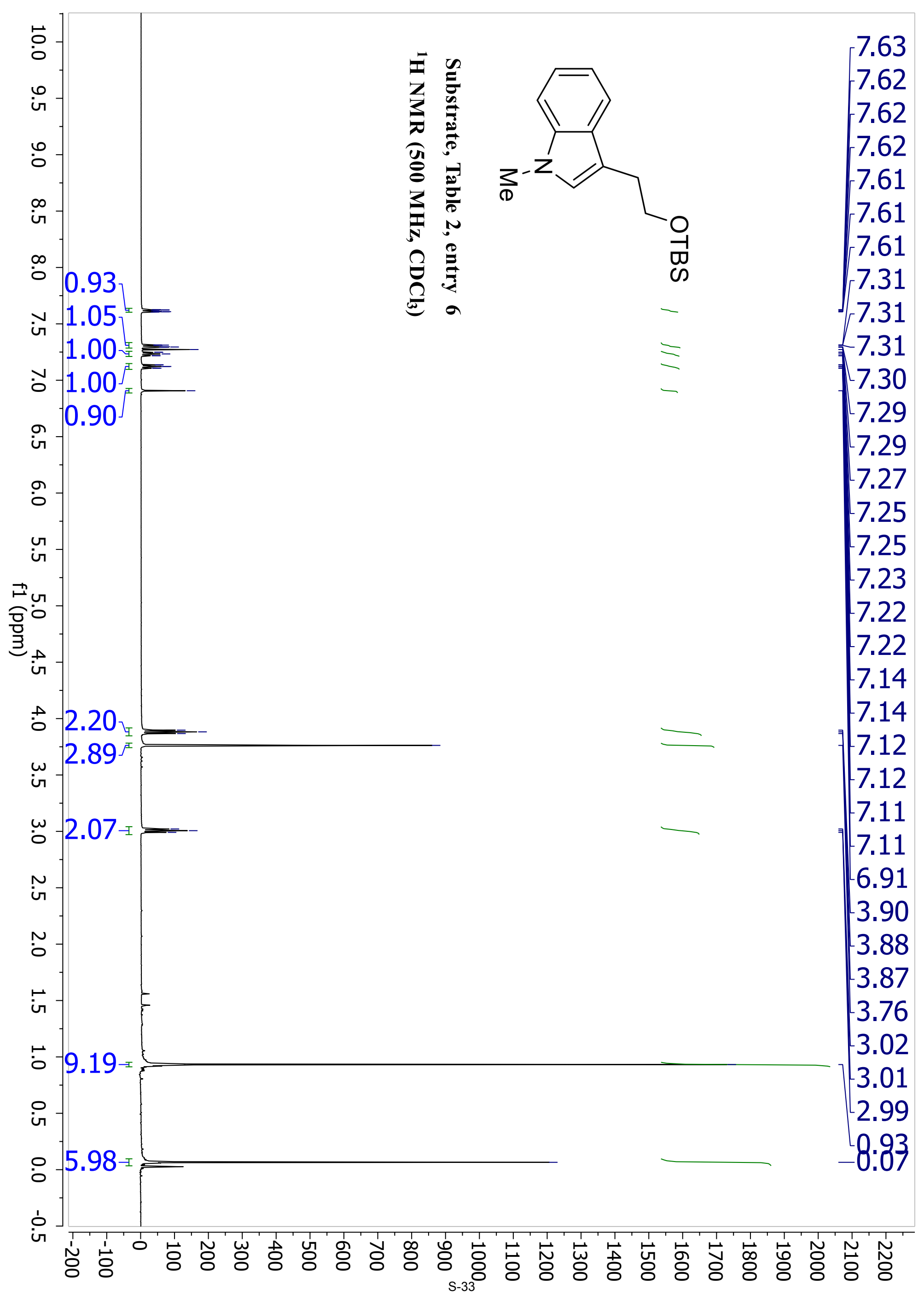




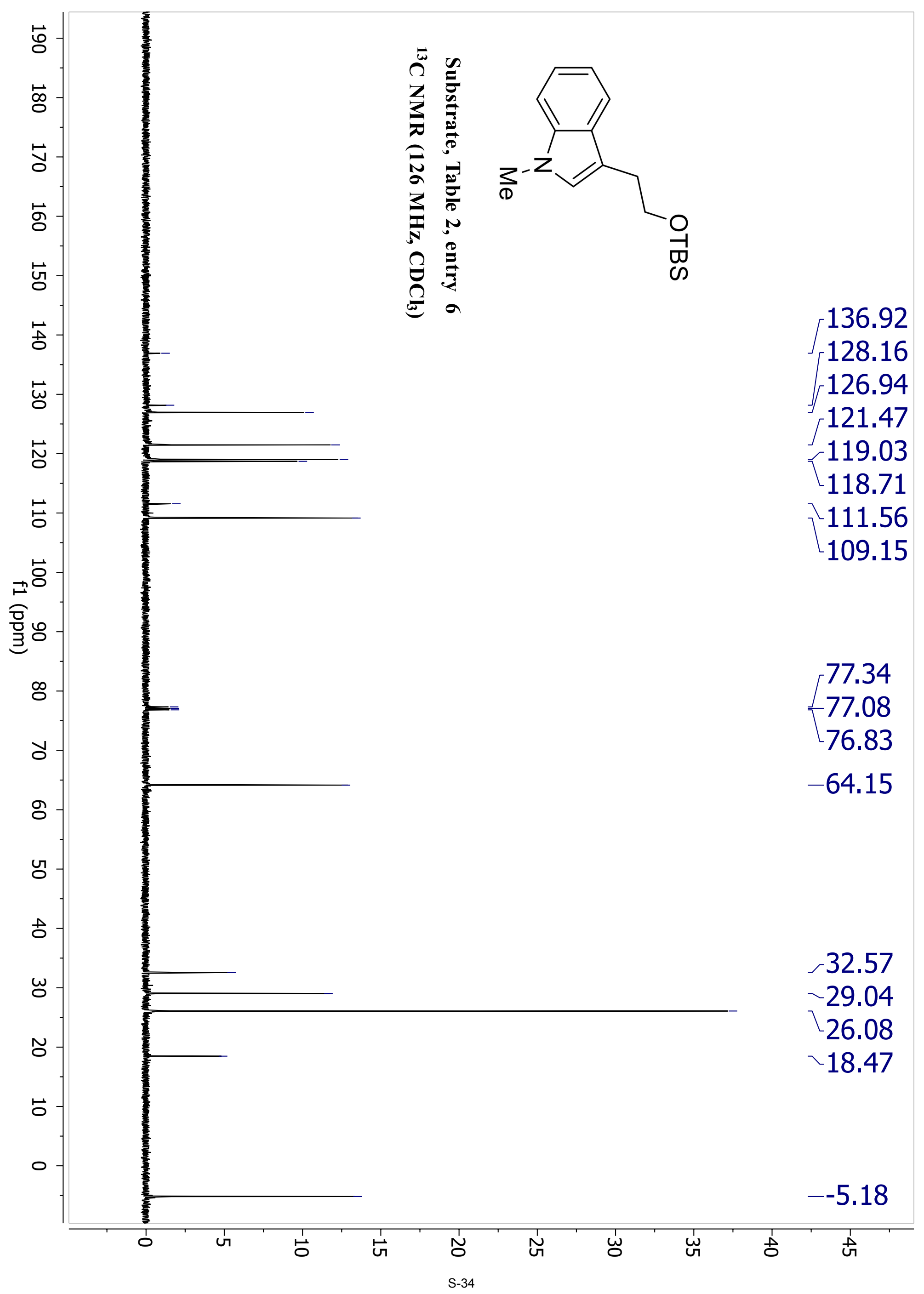




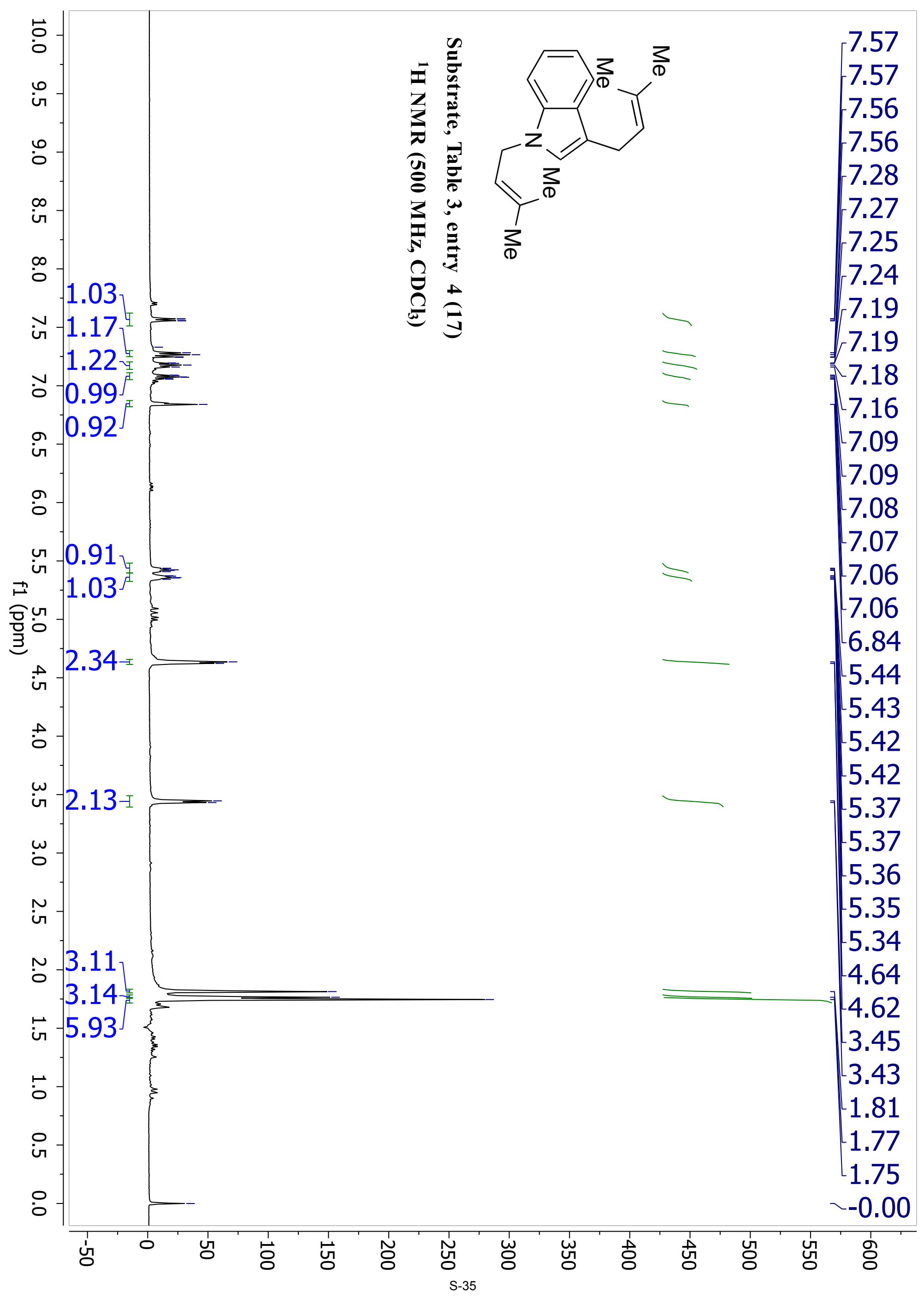




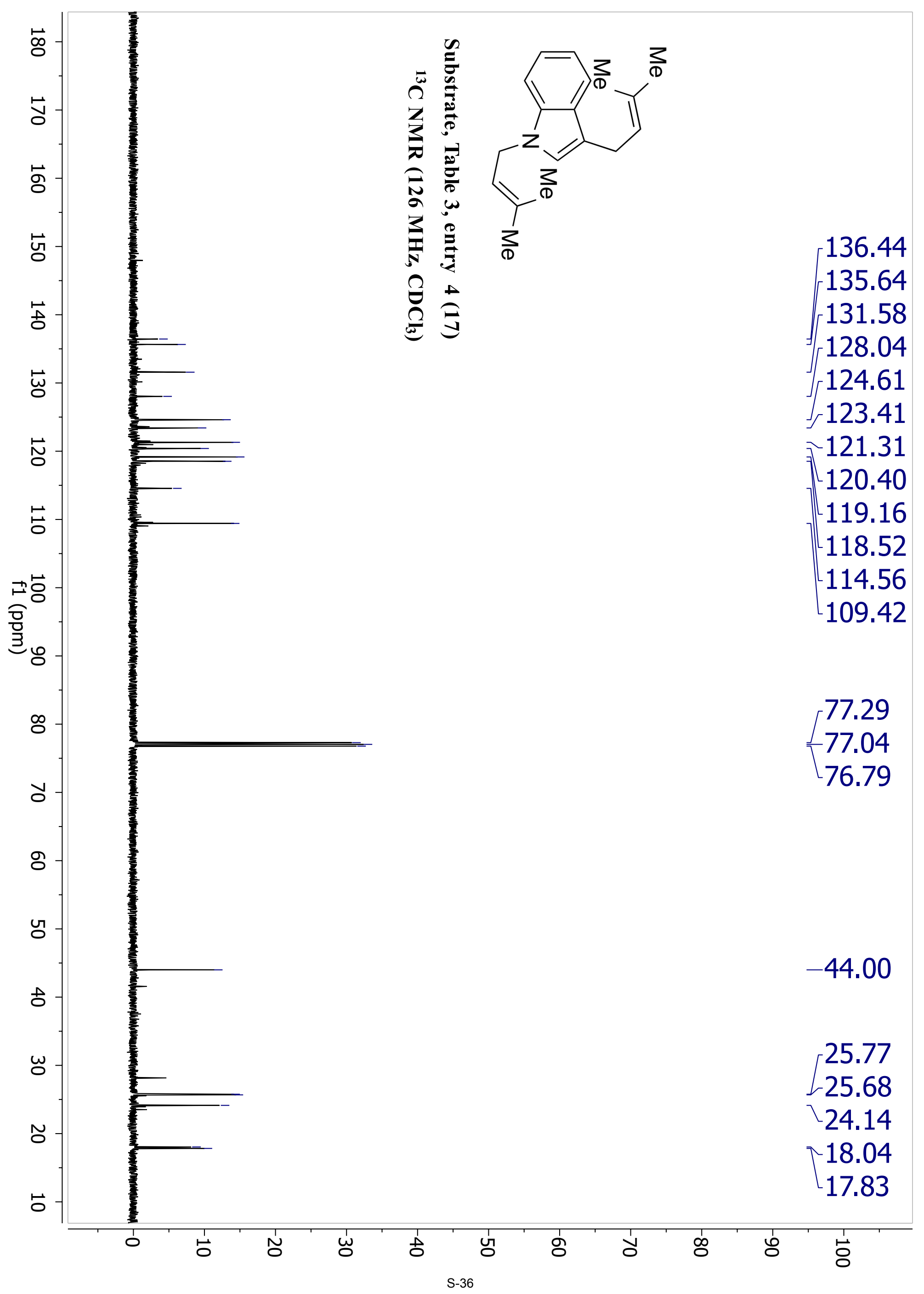




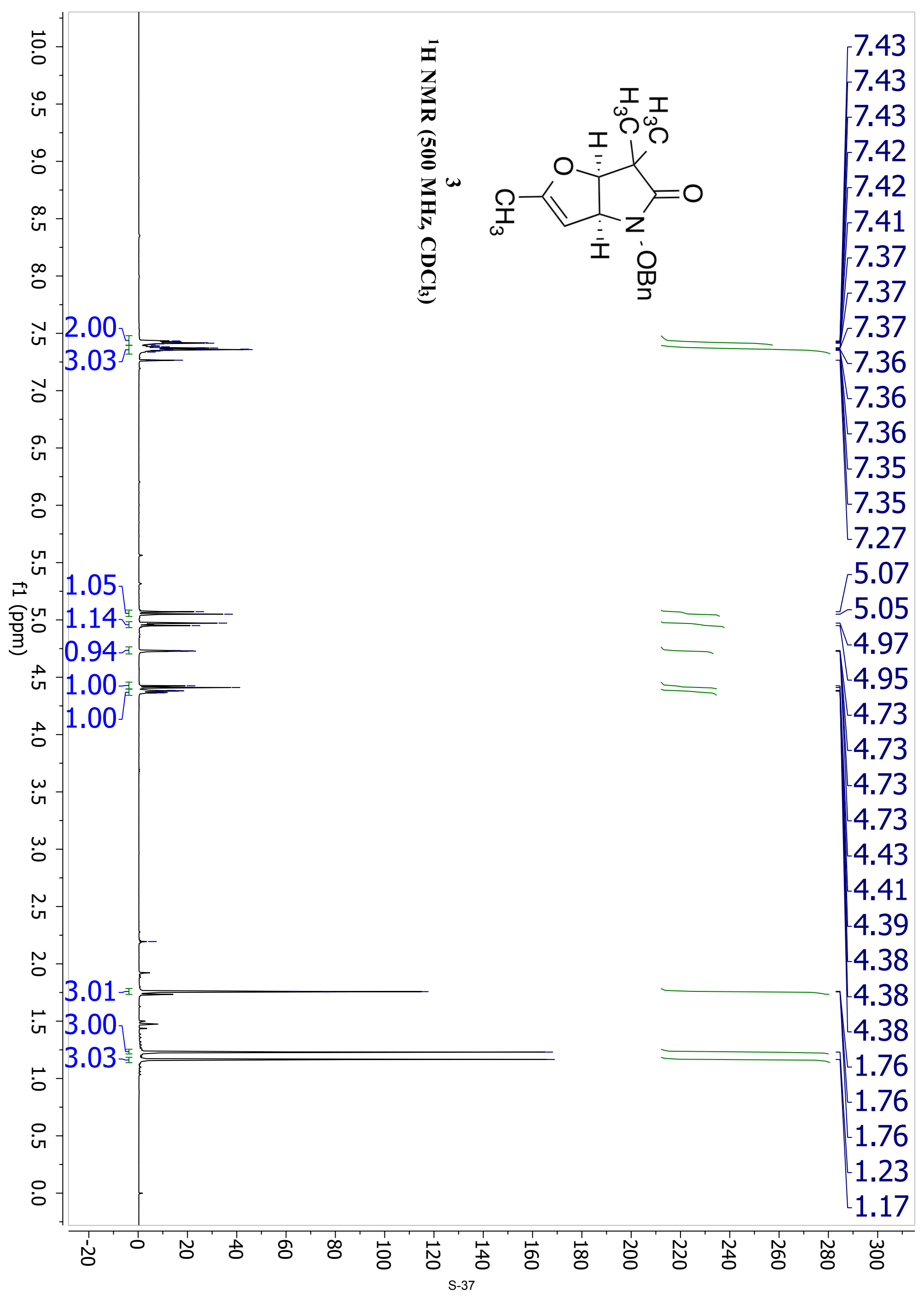




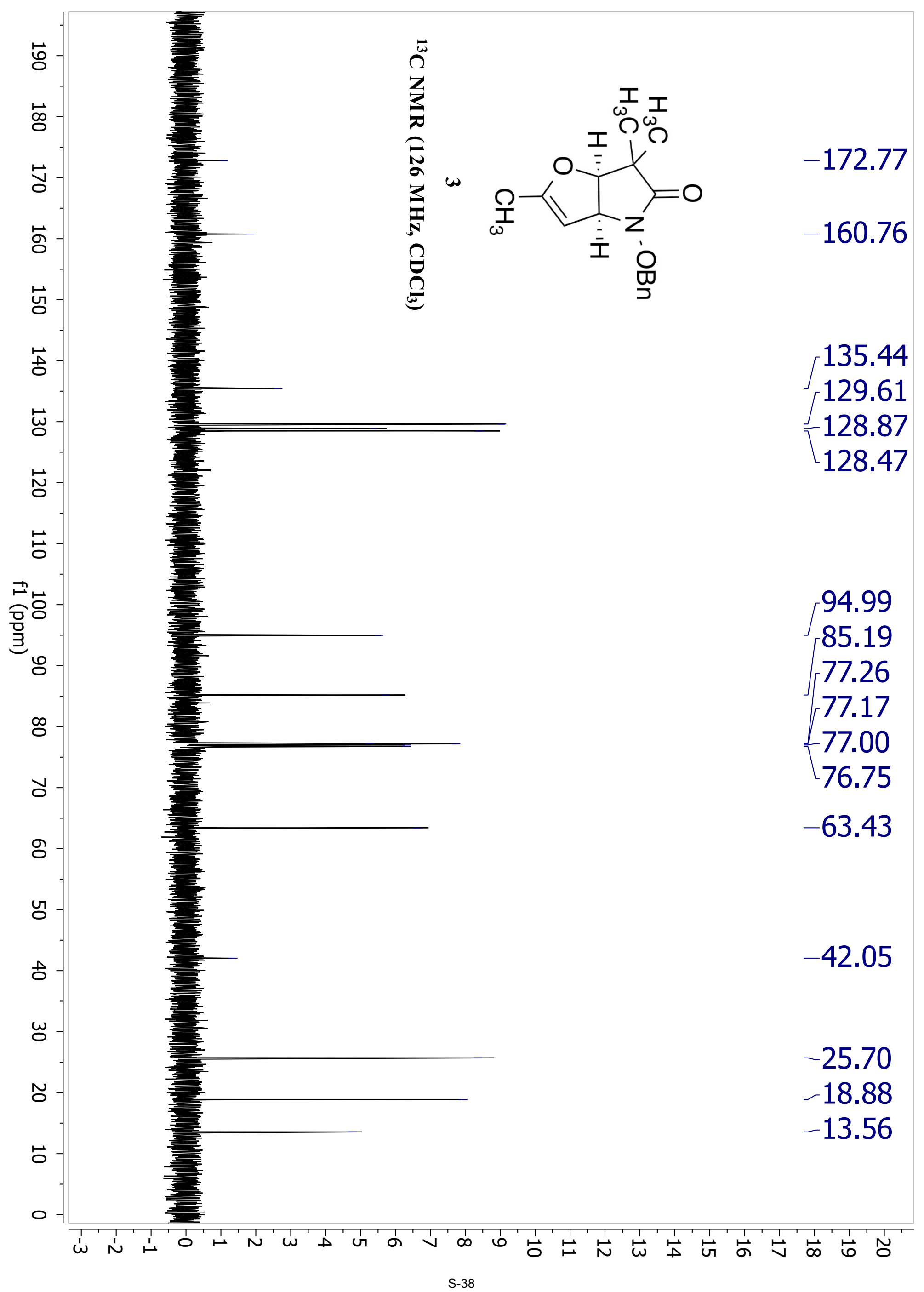




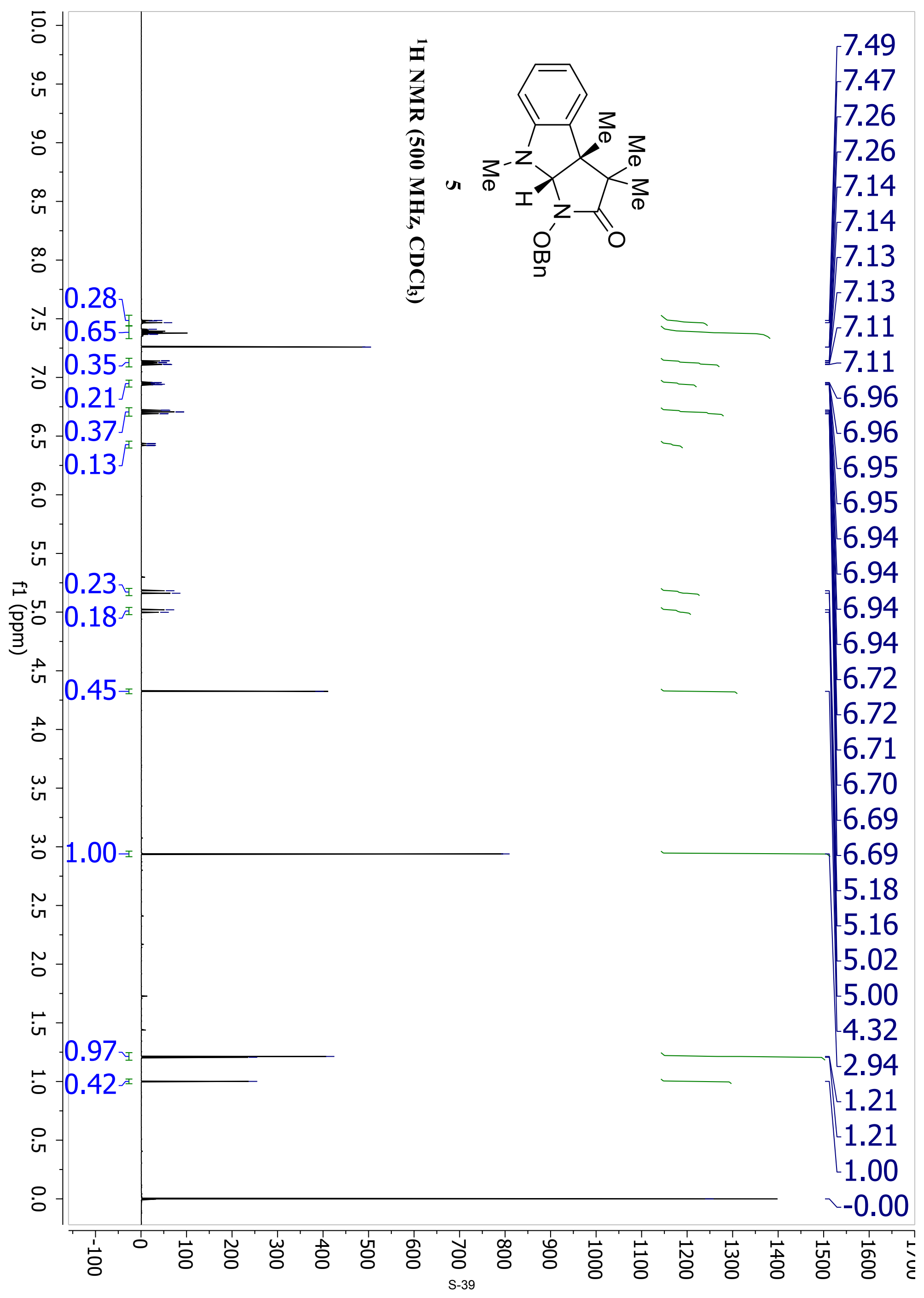




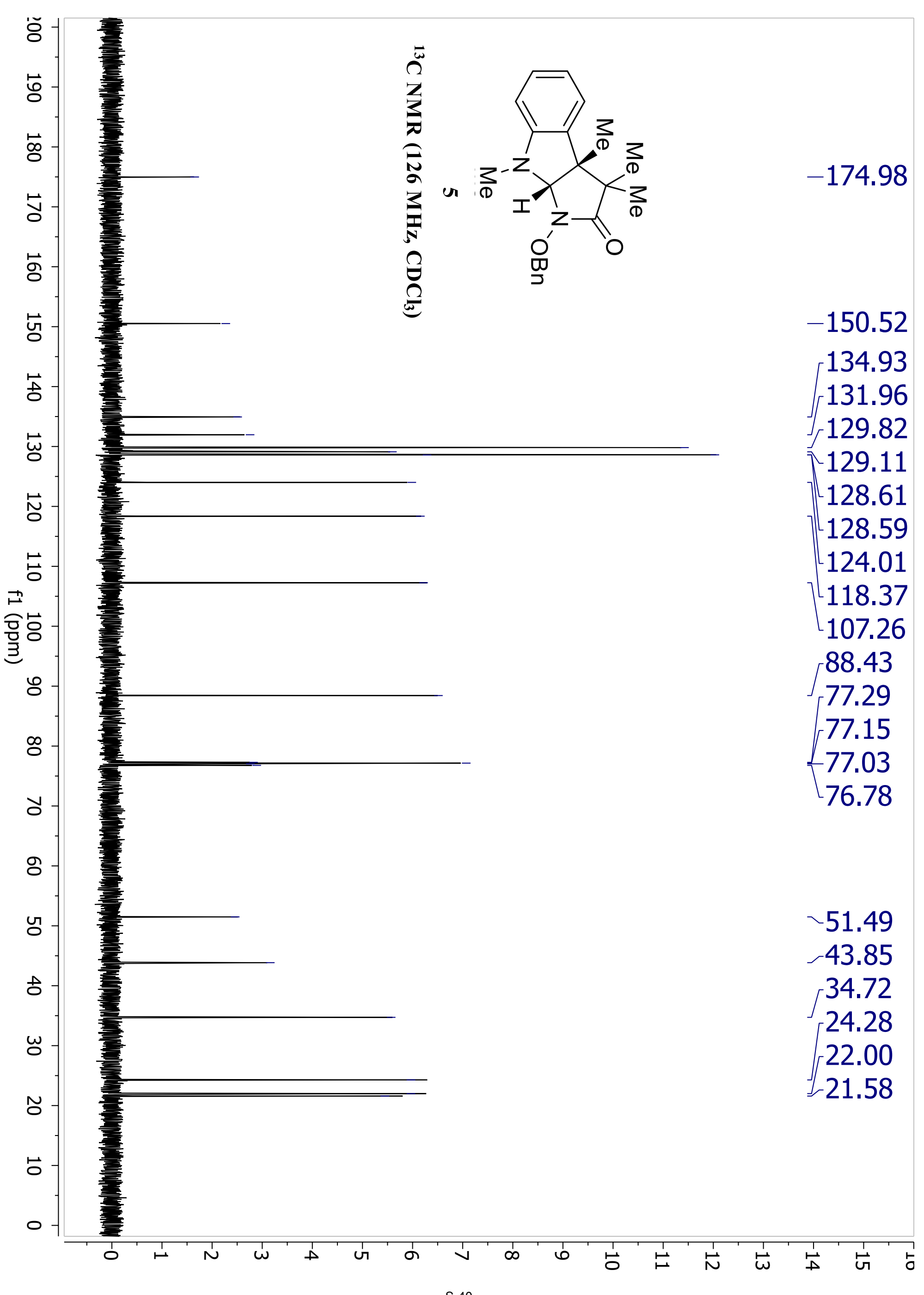




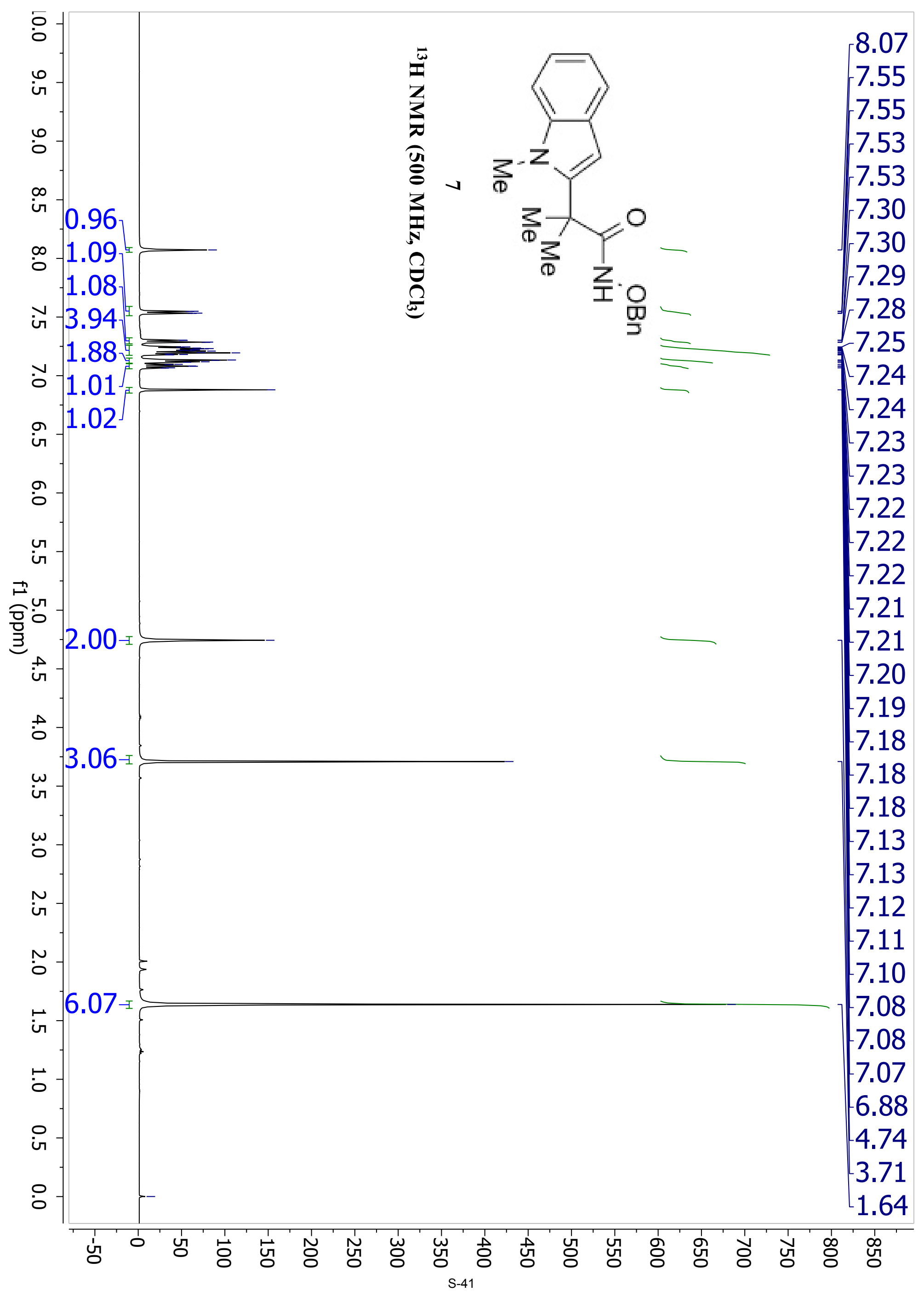




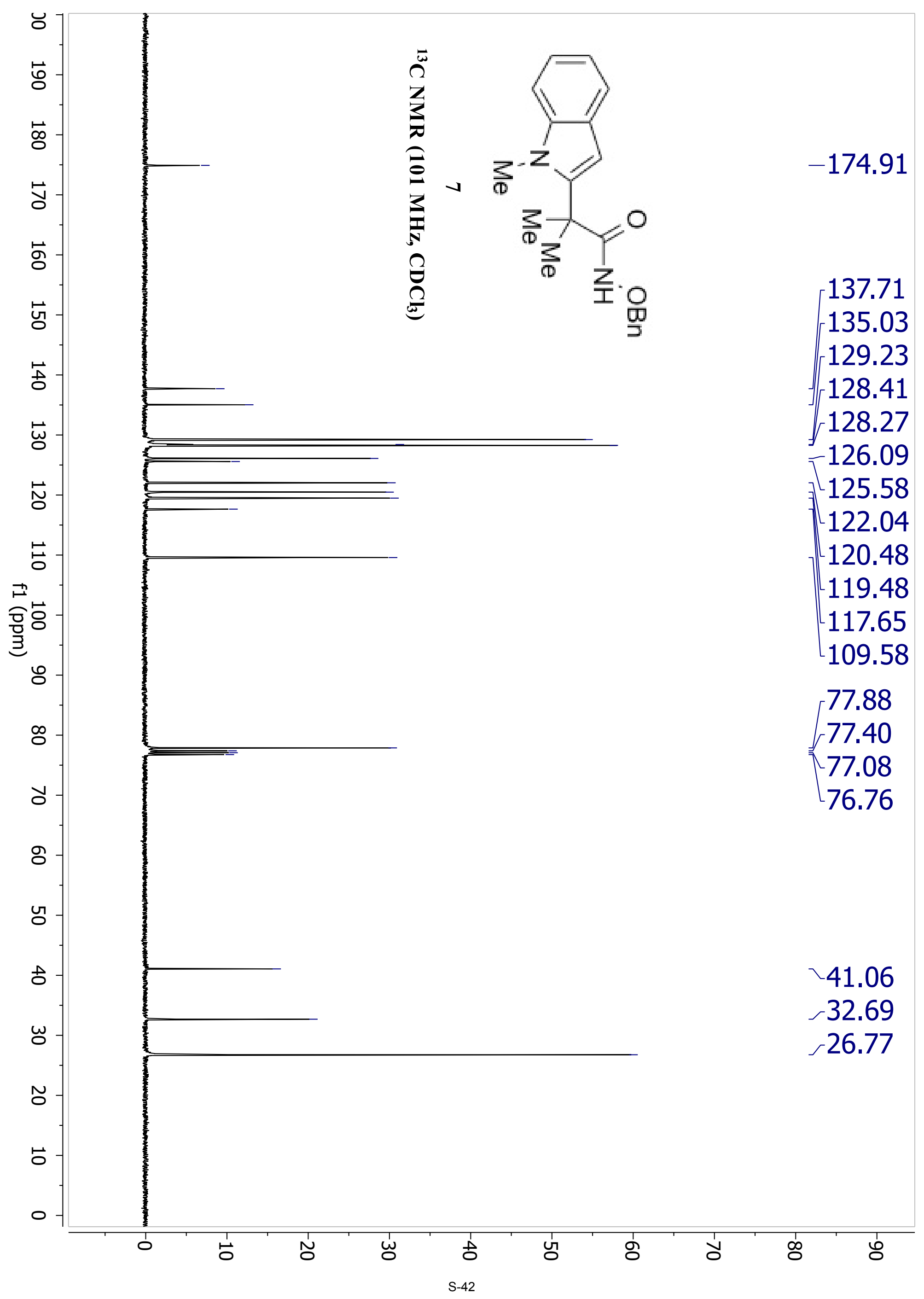




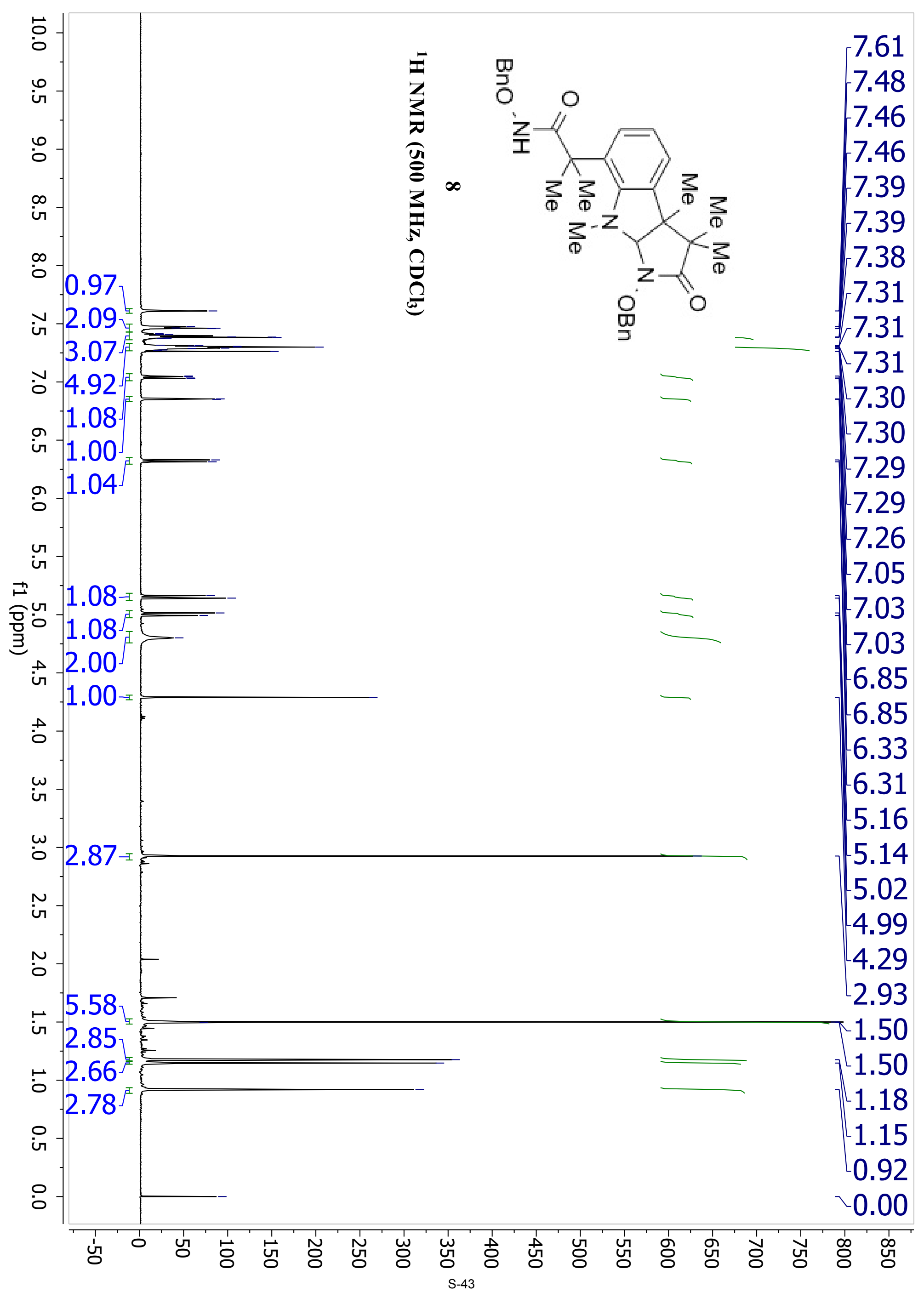




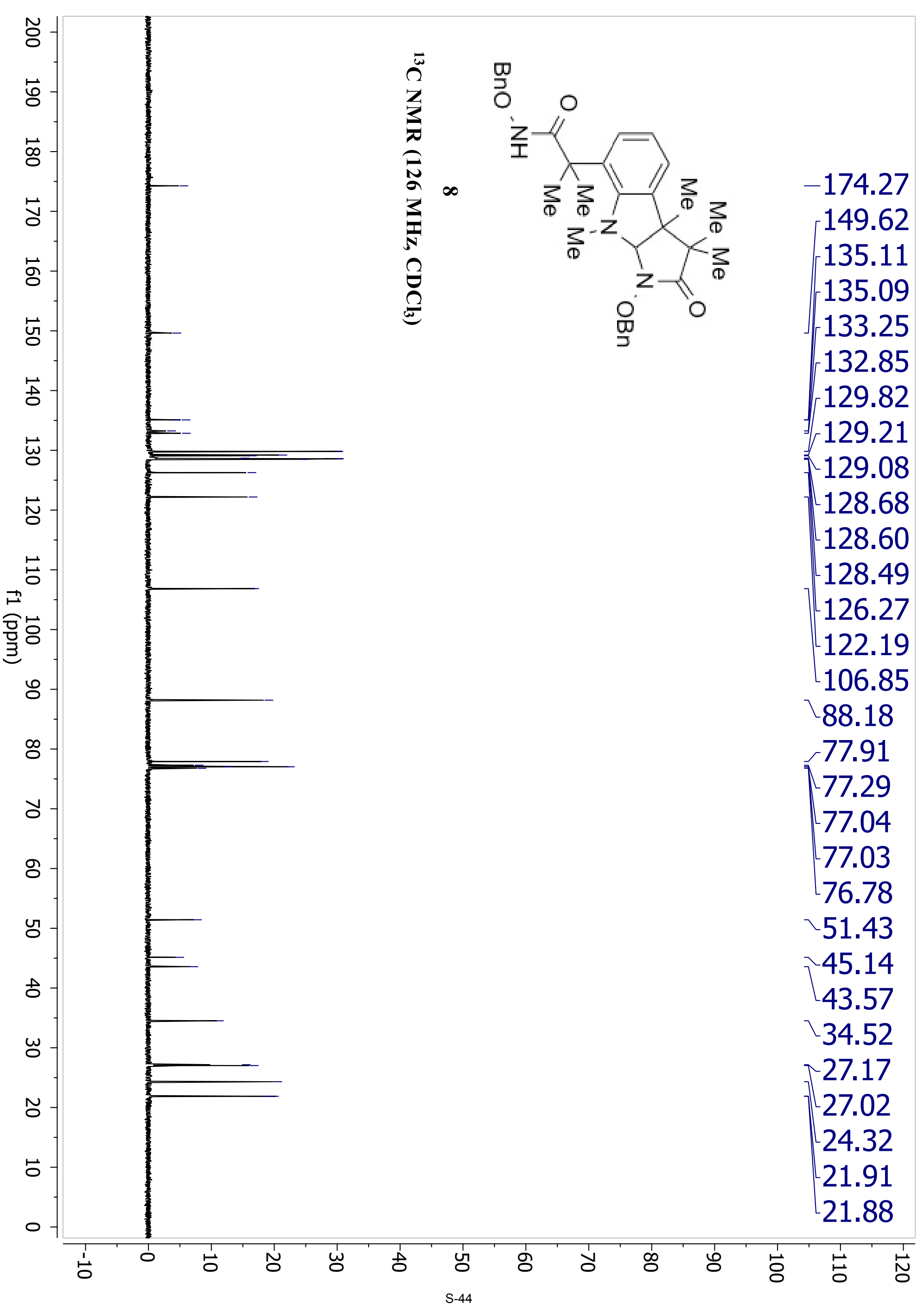




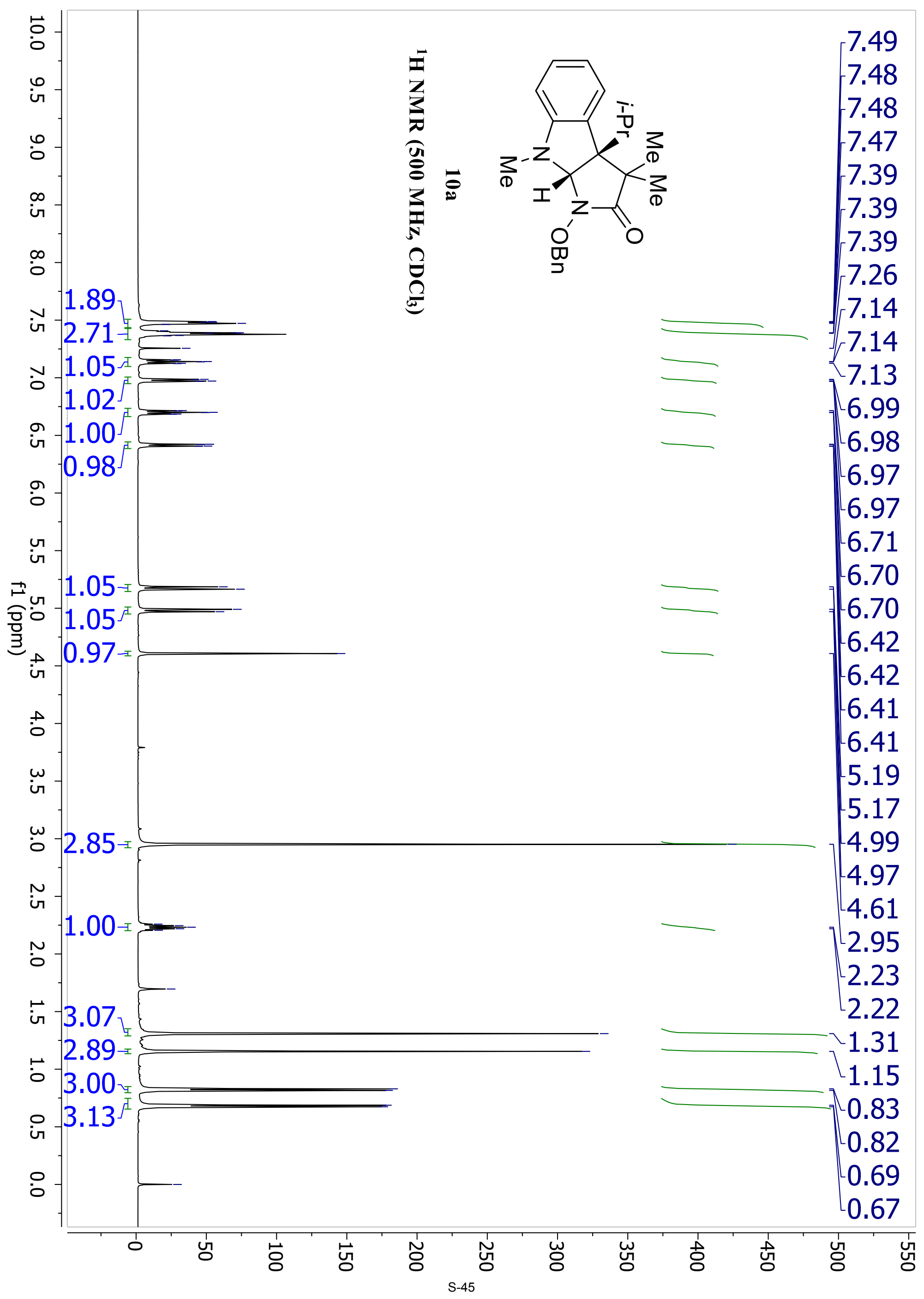




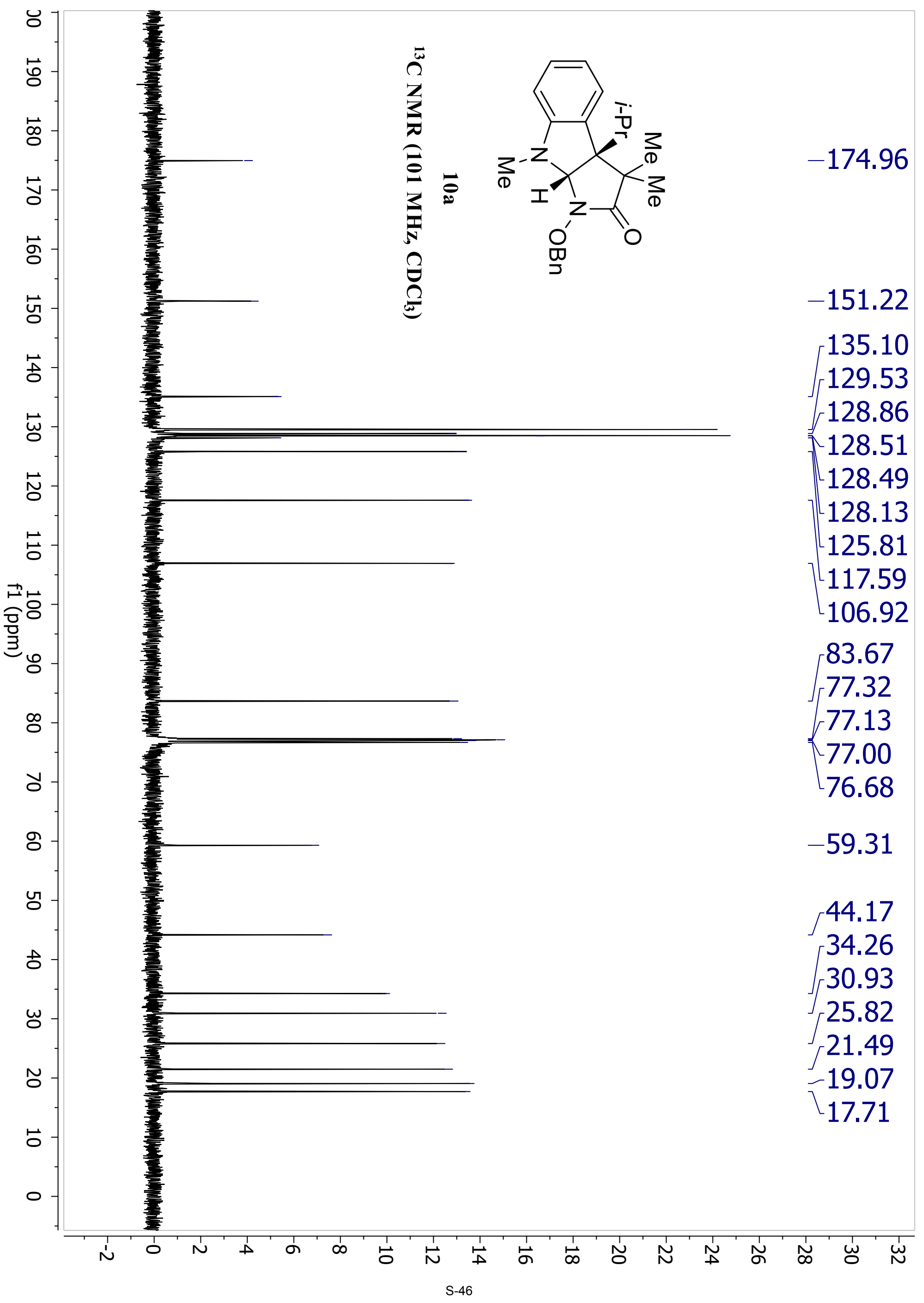




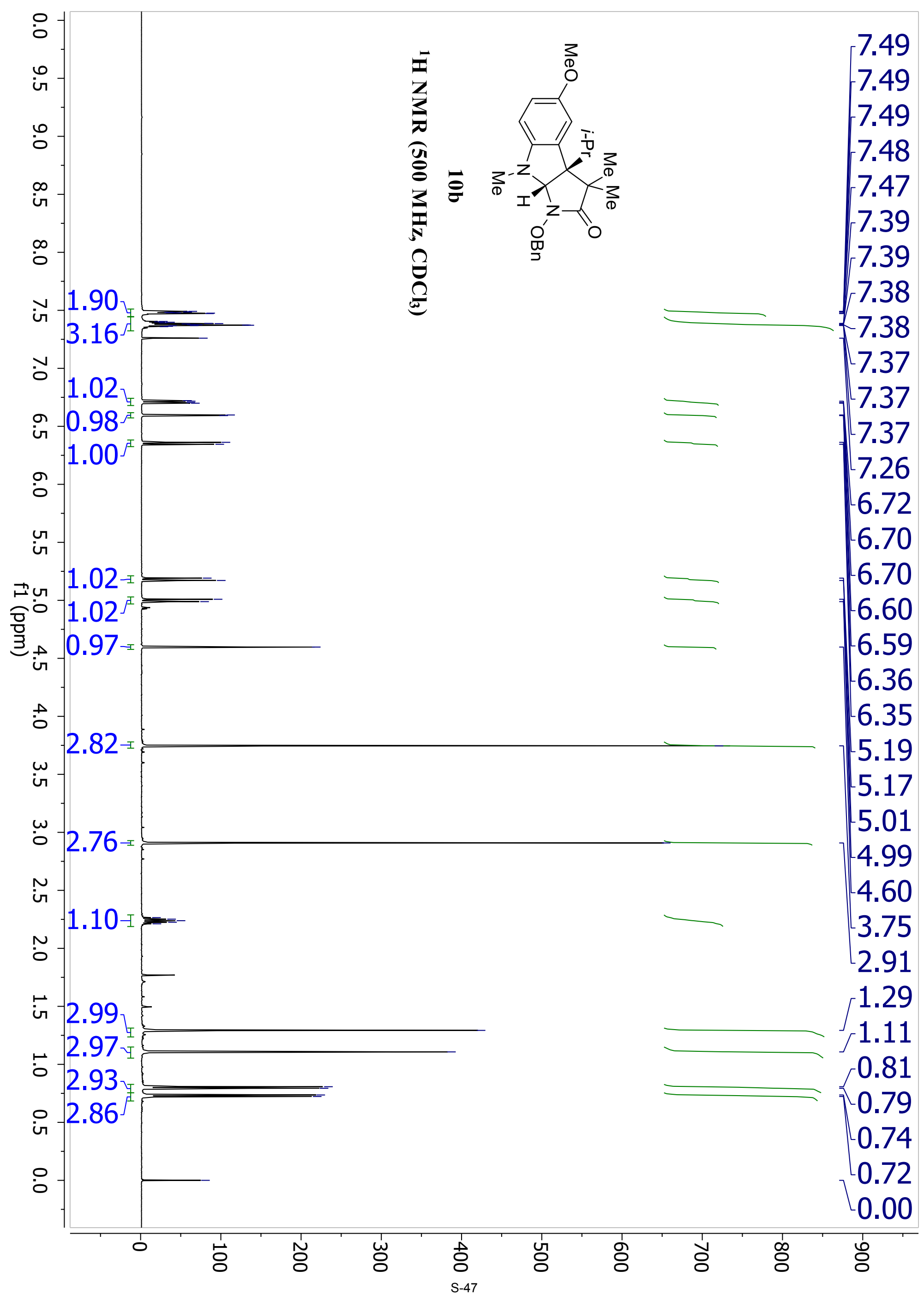




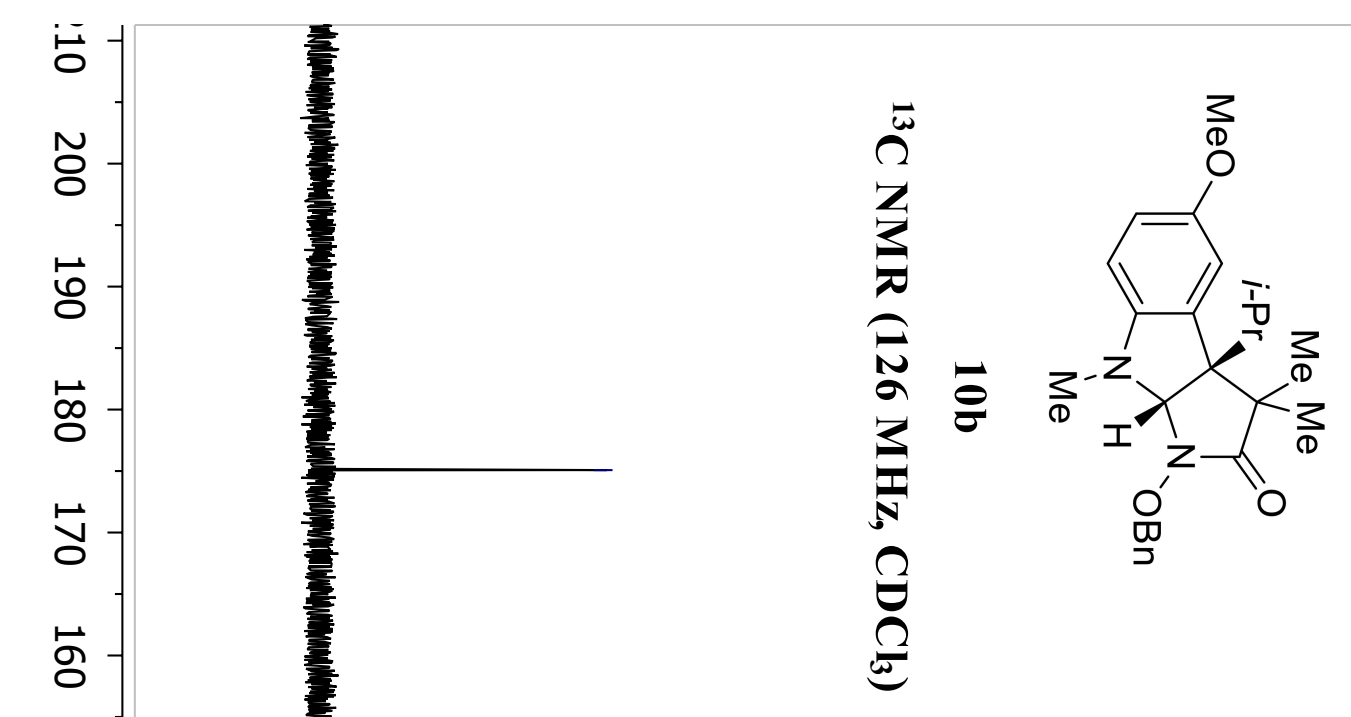

ㄴ

0 


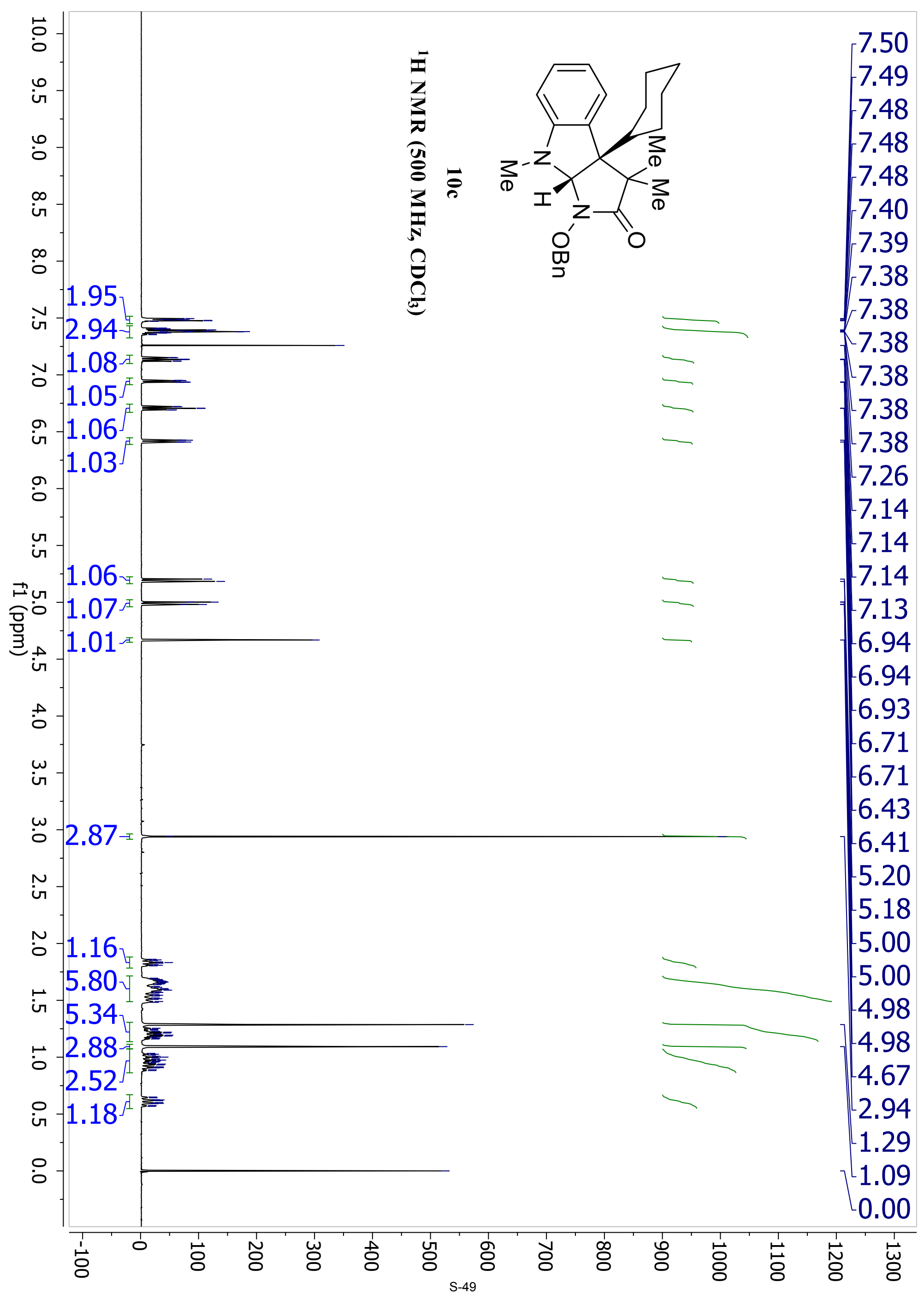




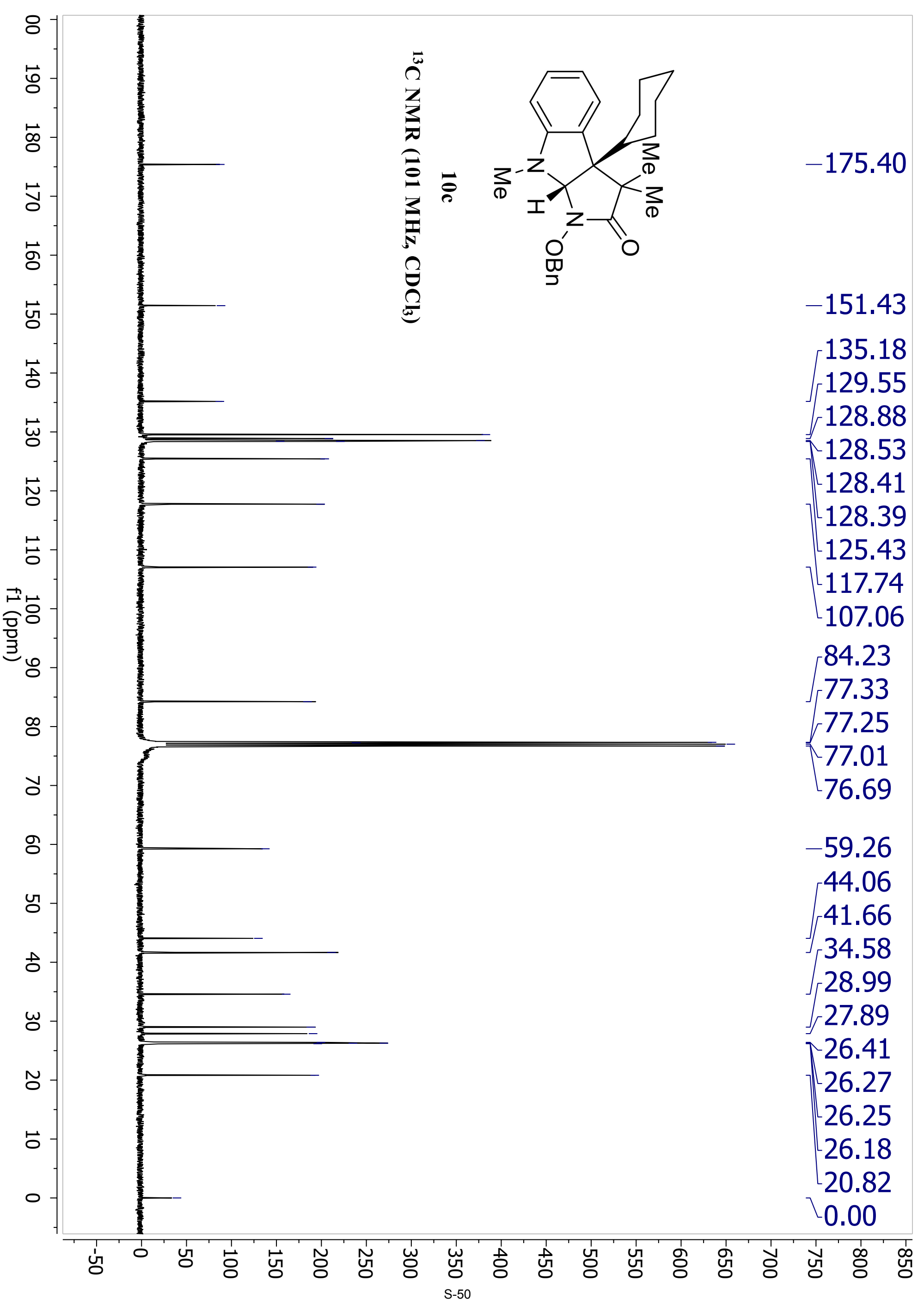




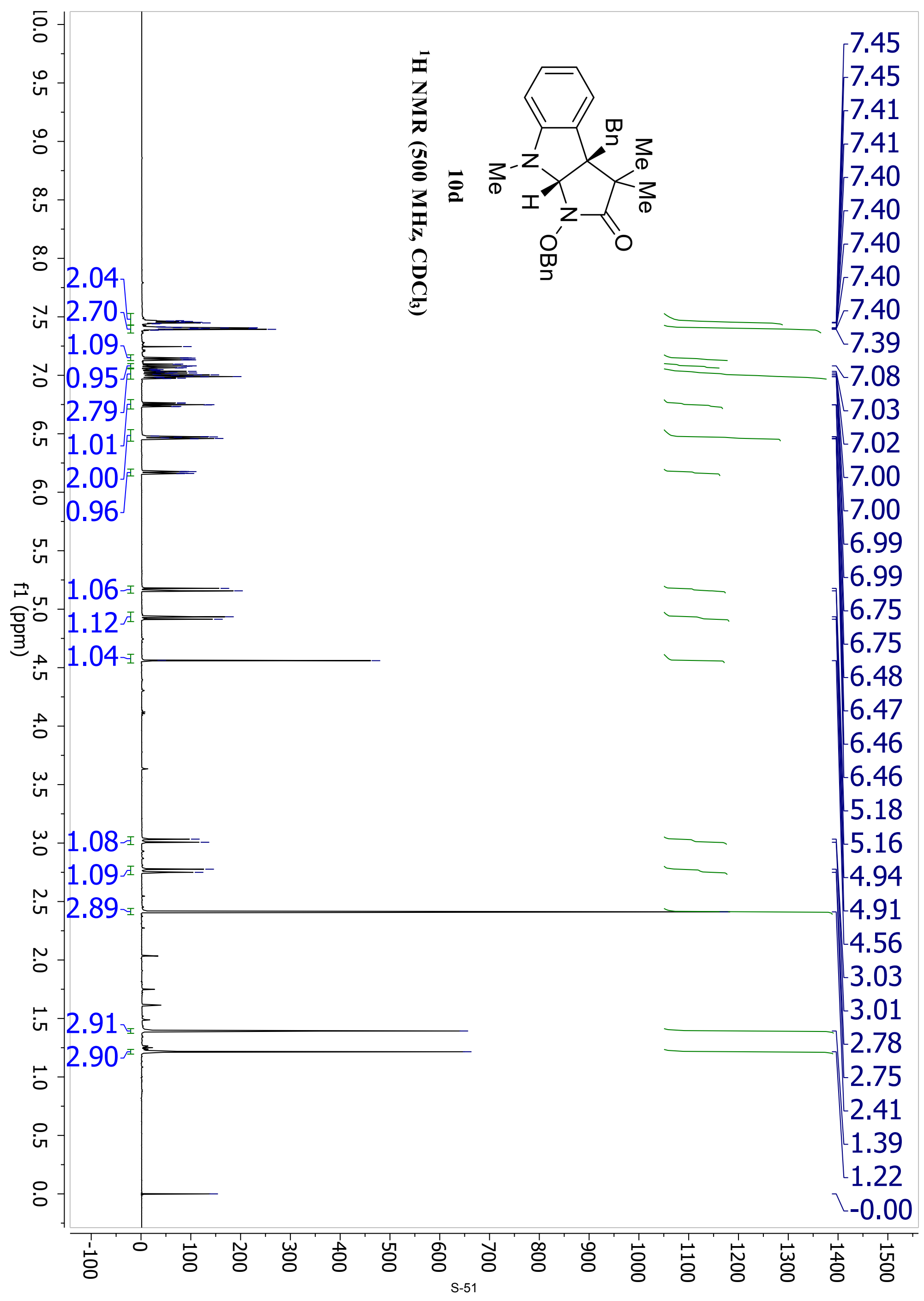




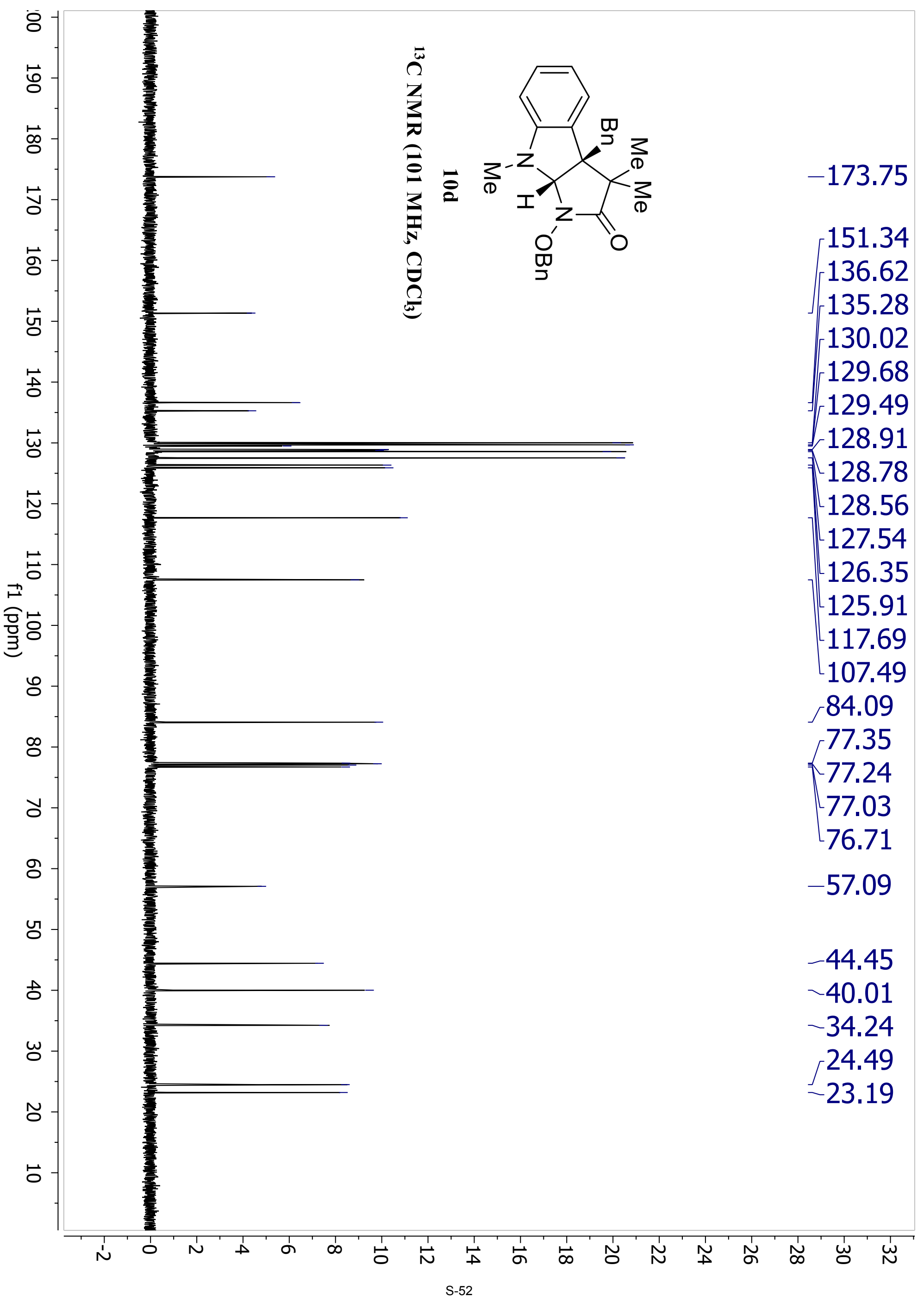




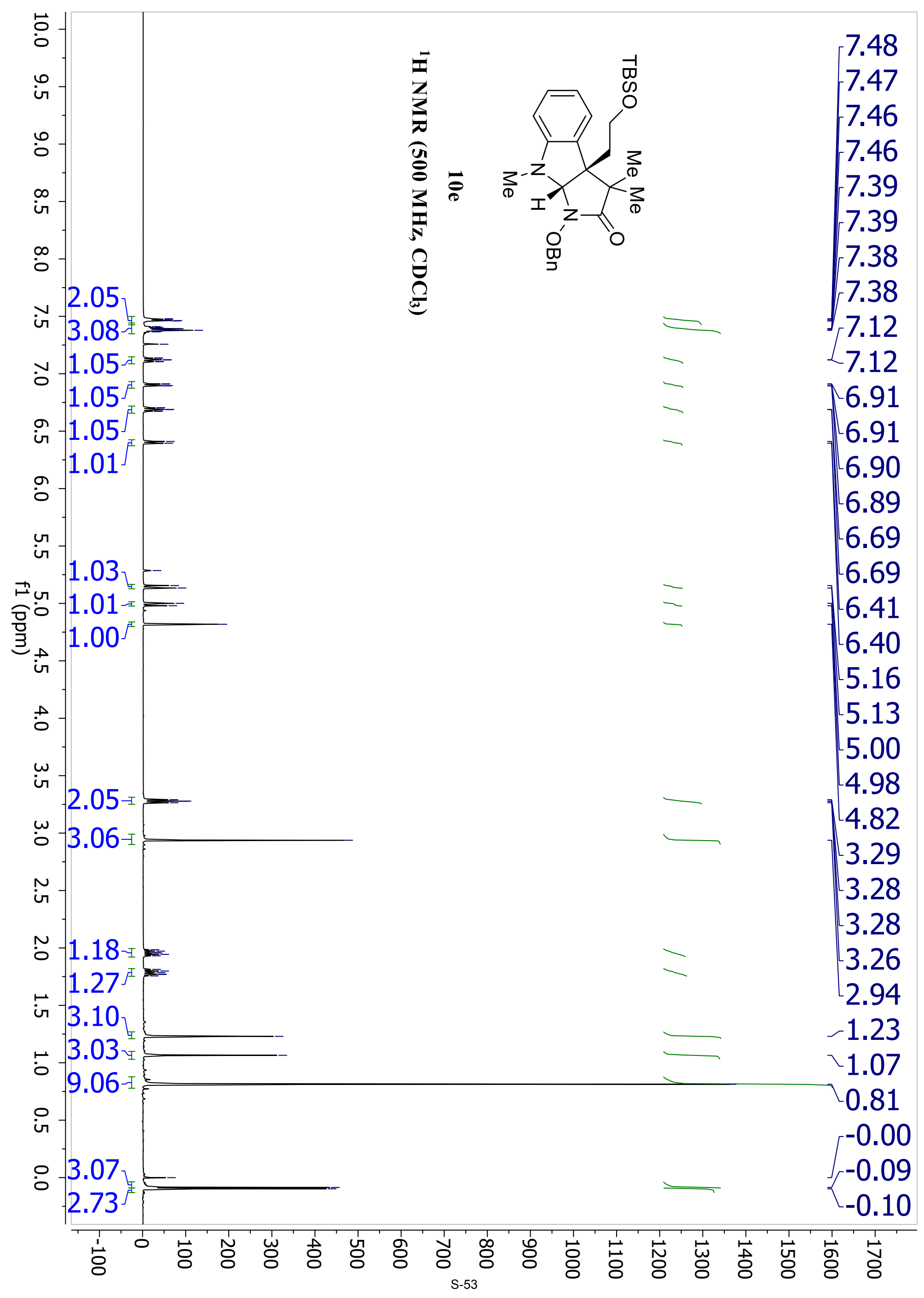




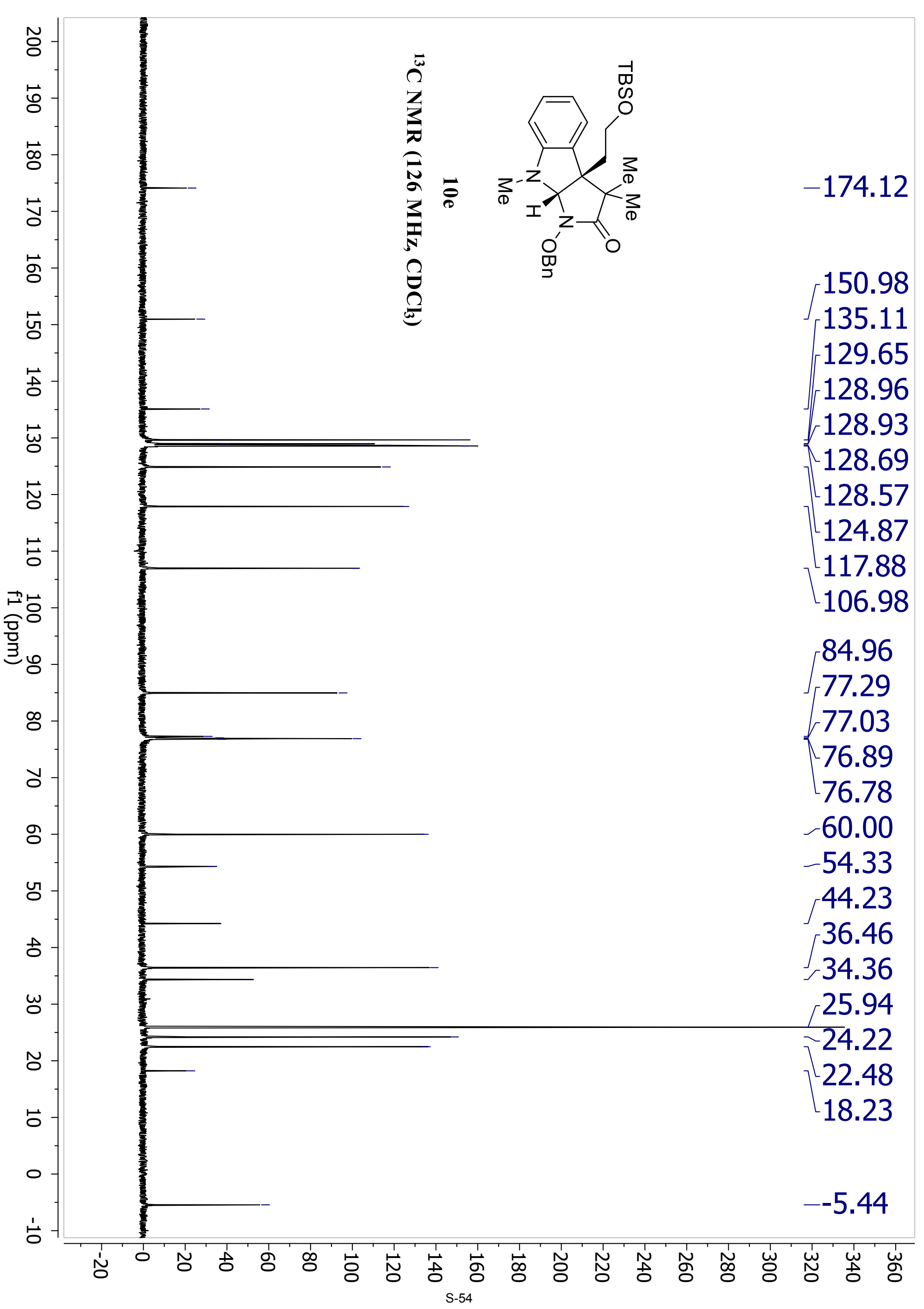




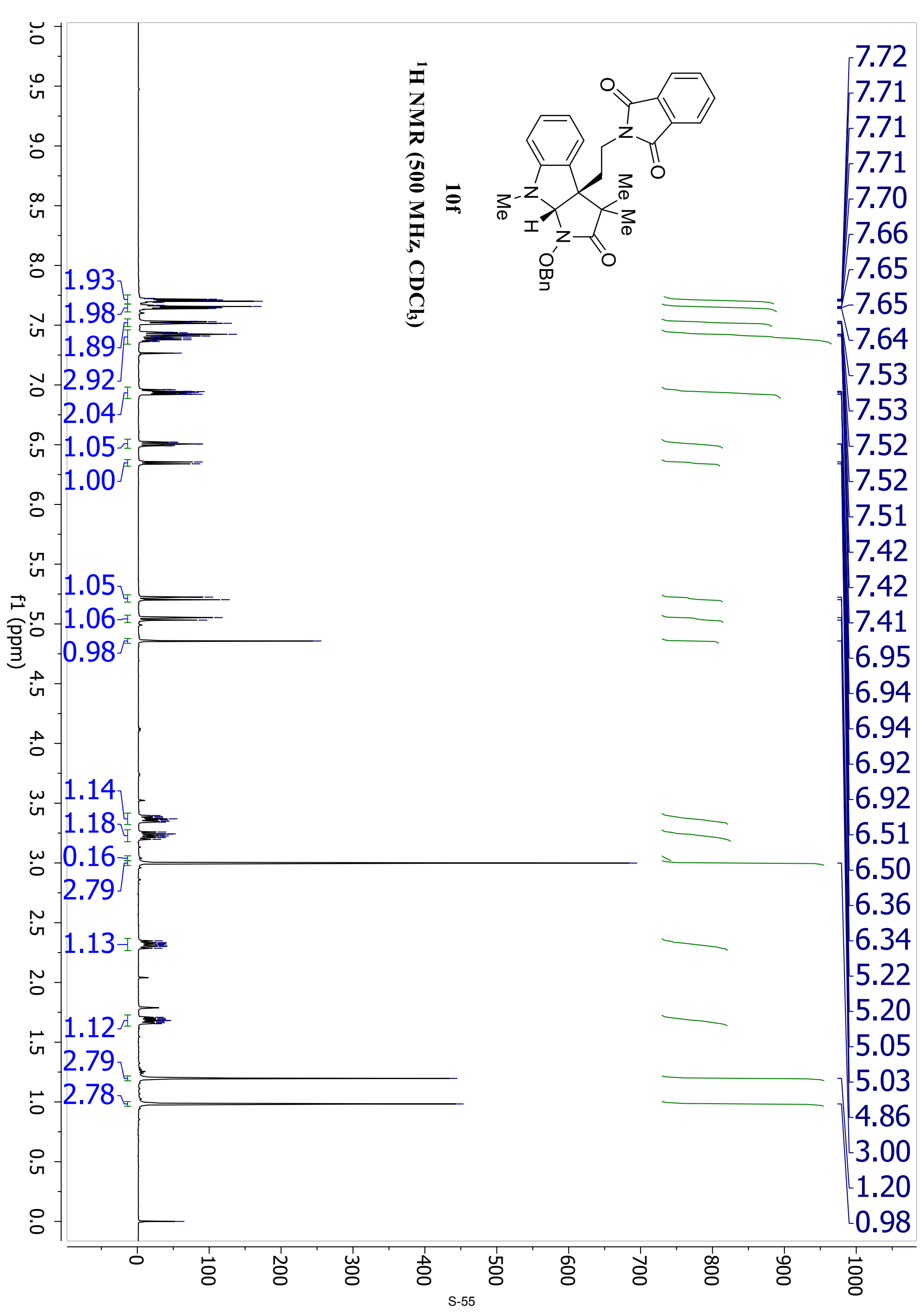




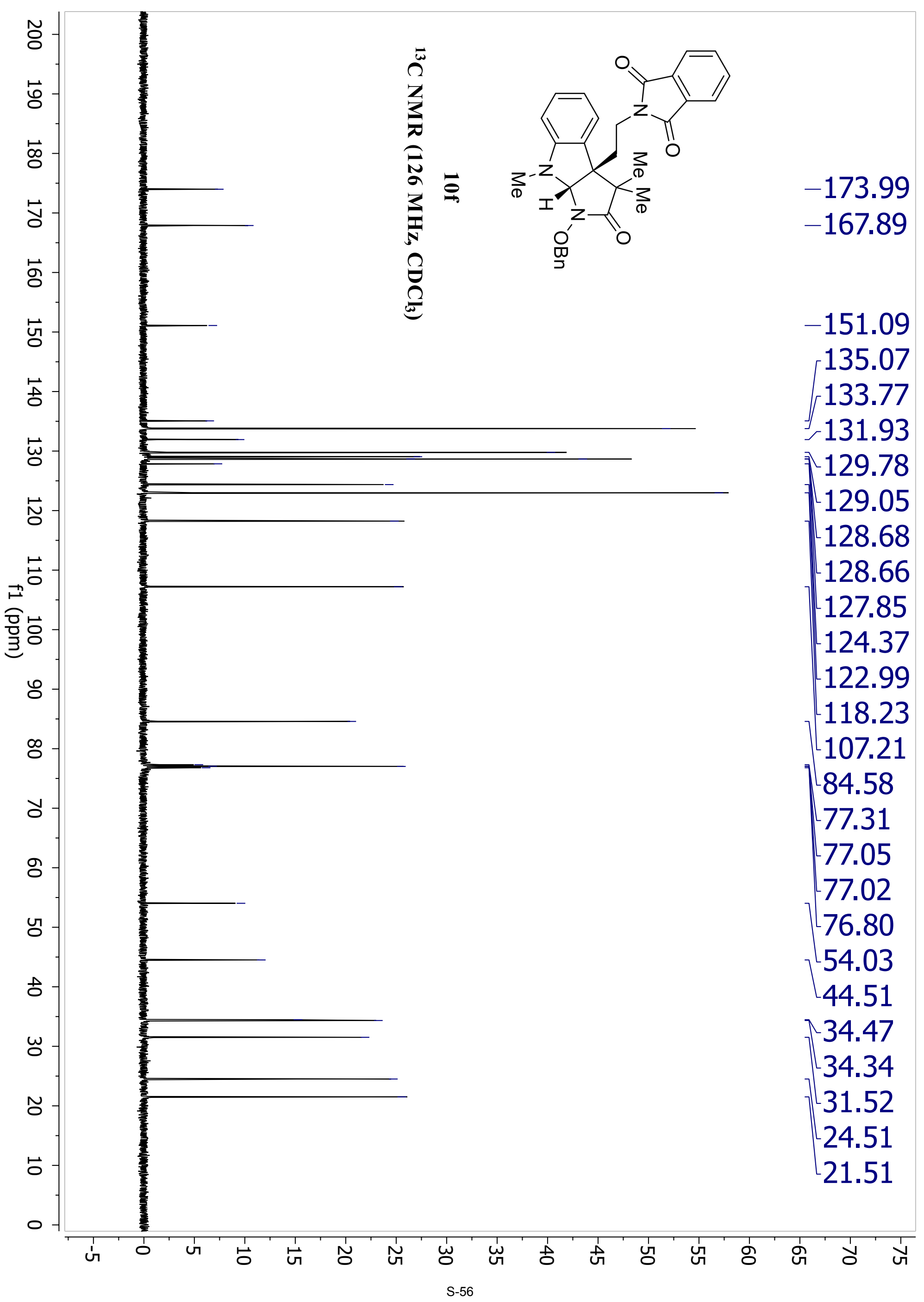




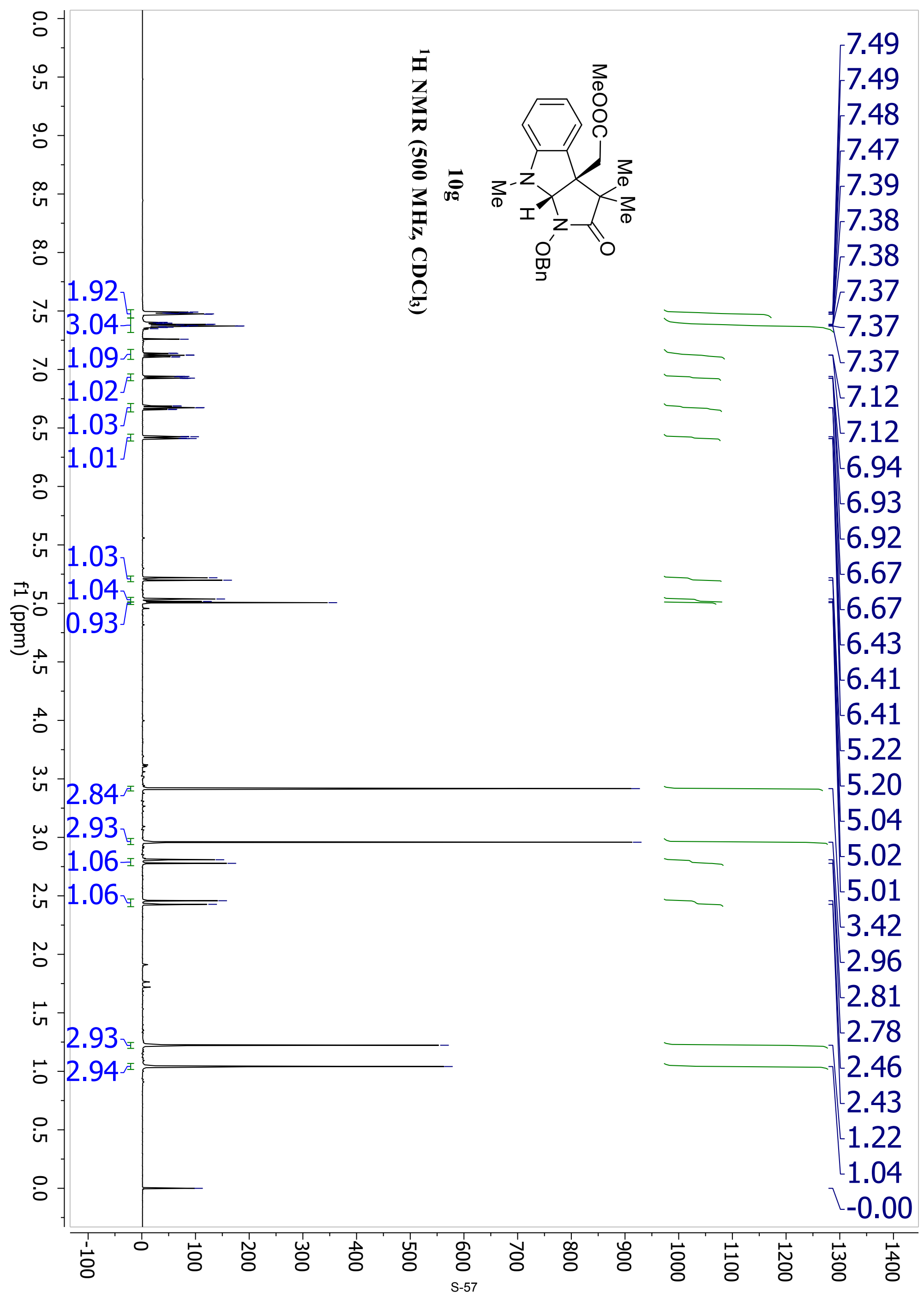




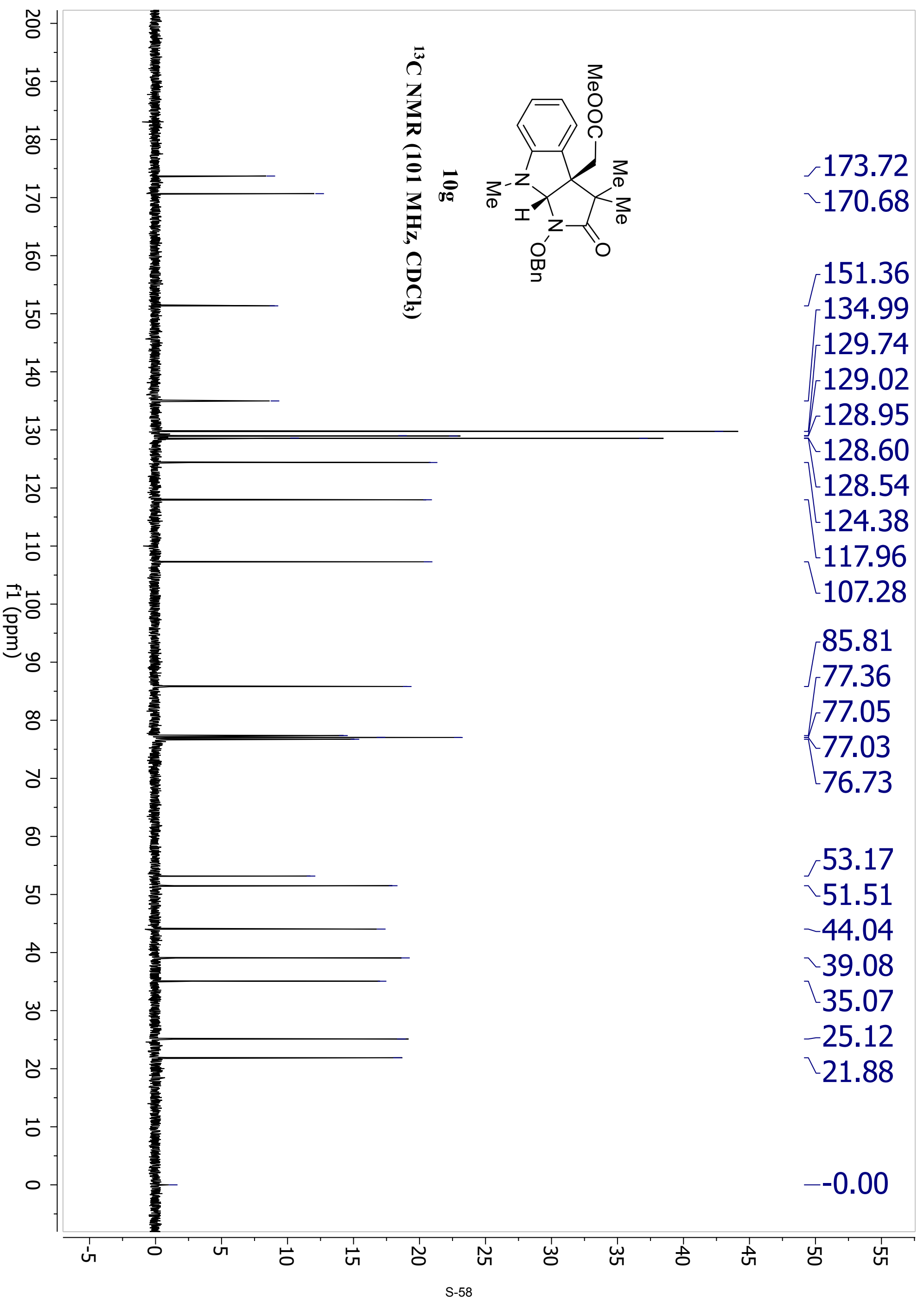




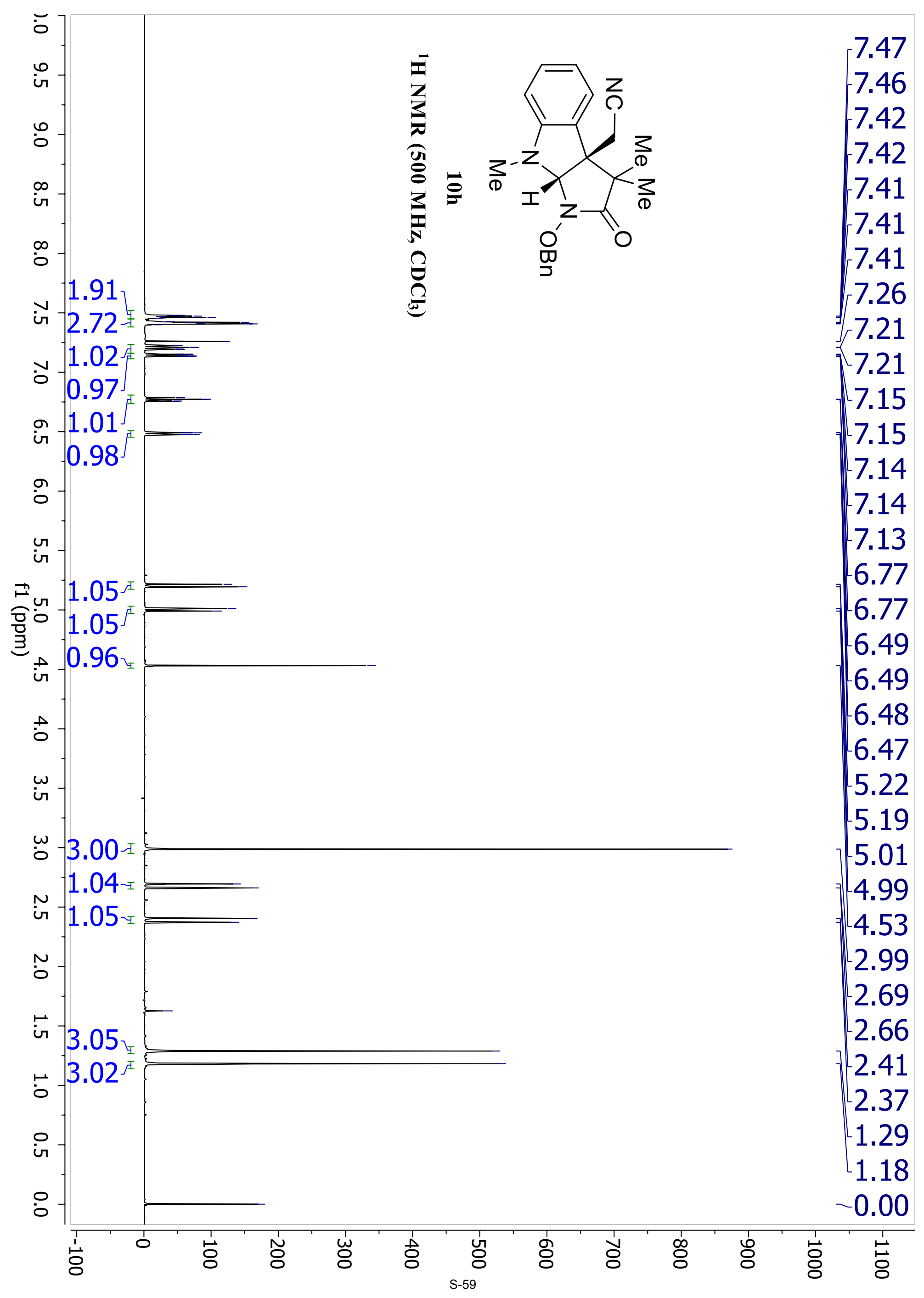




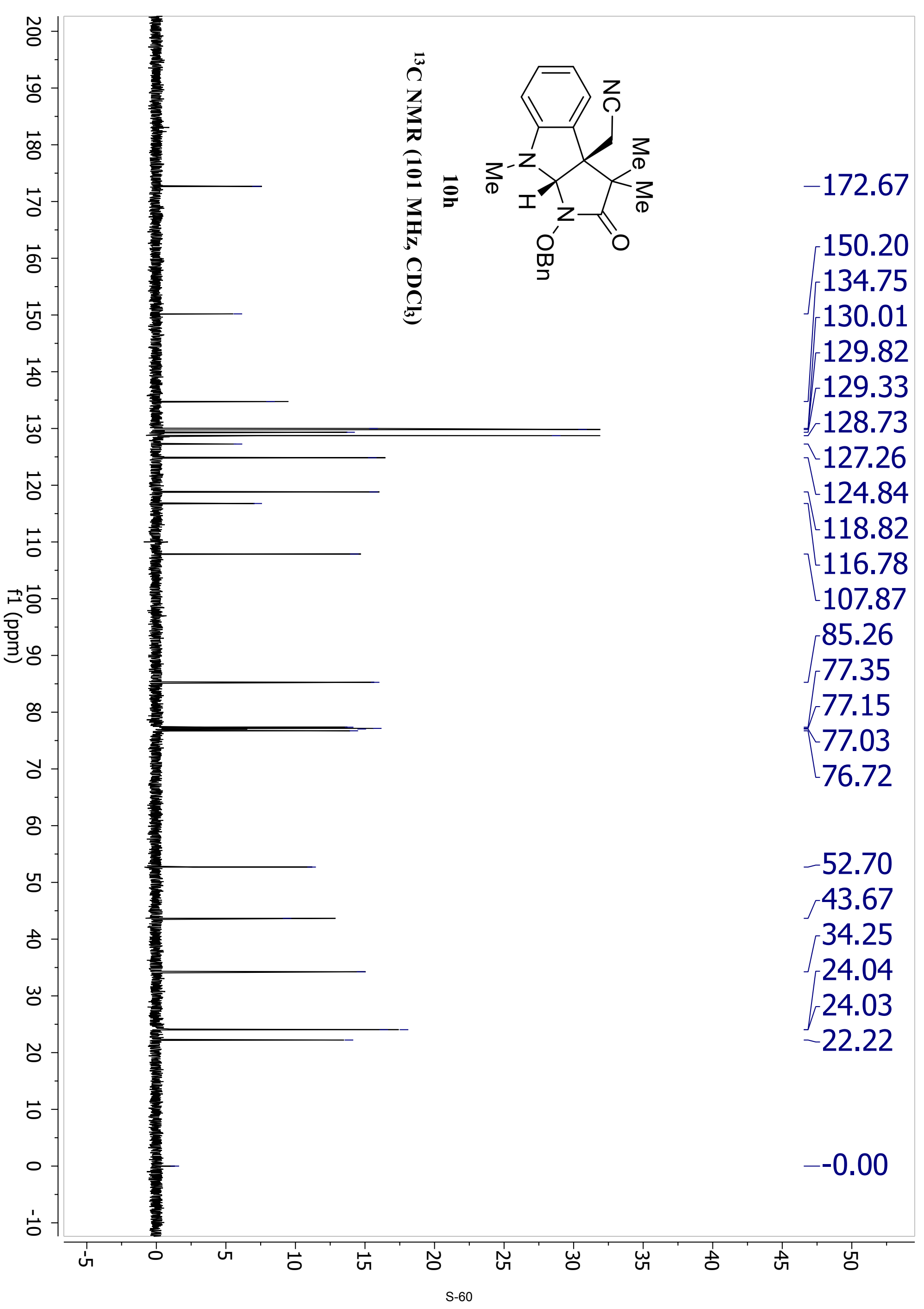




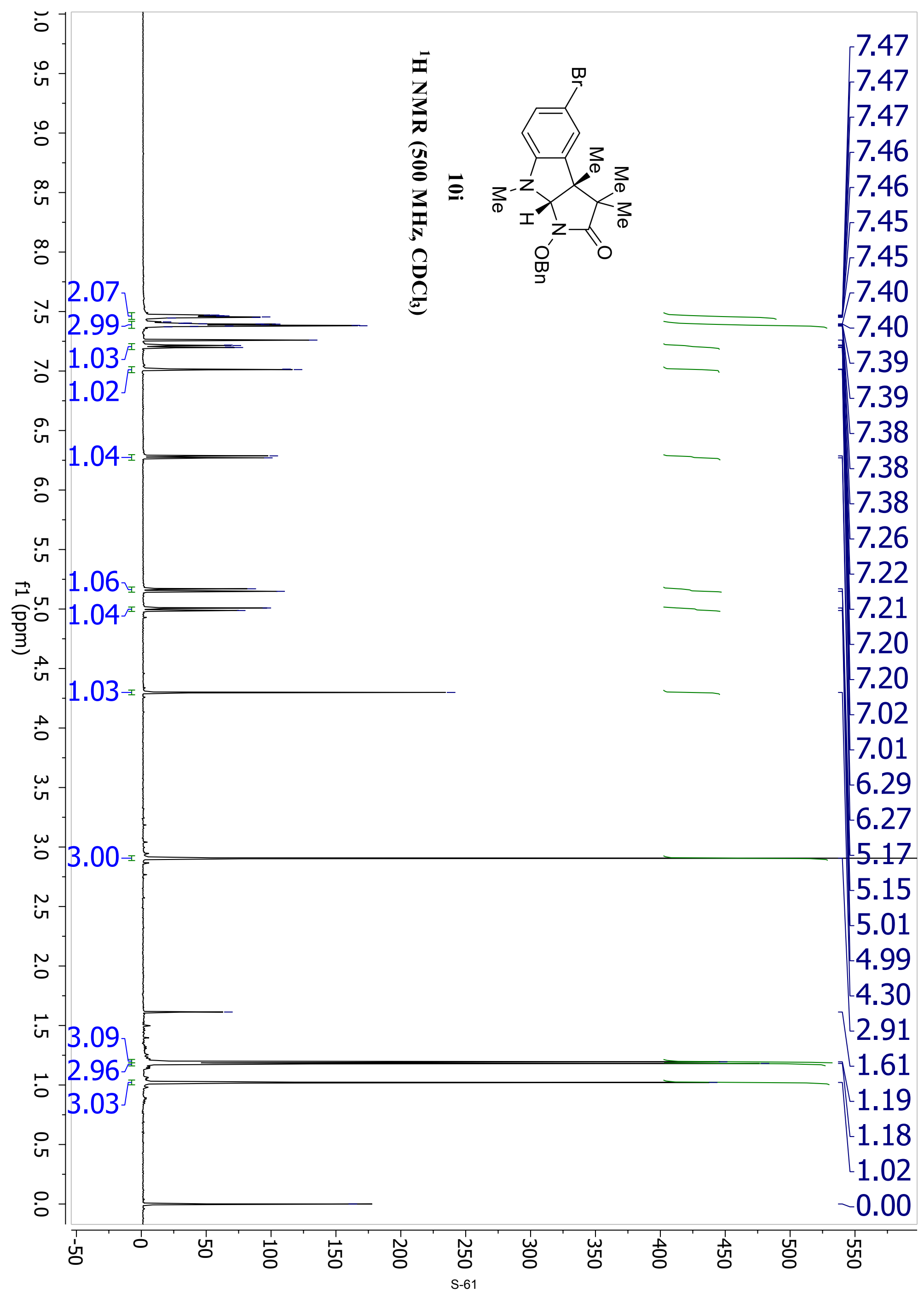




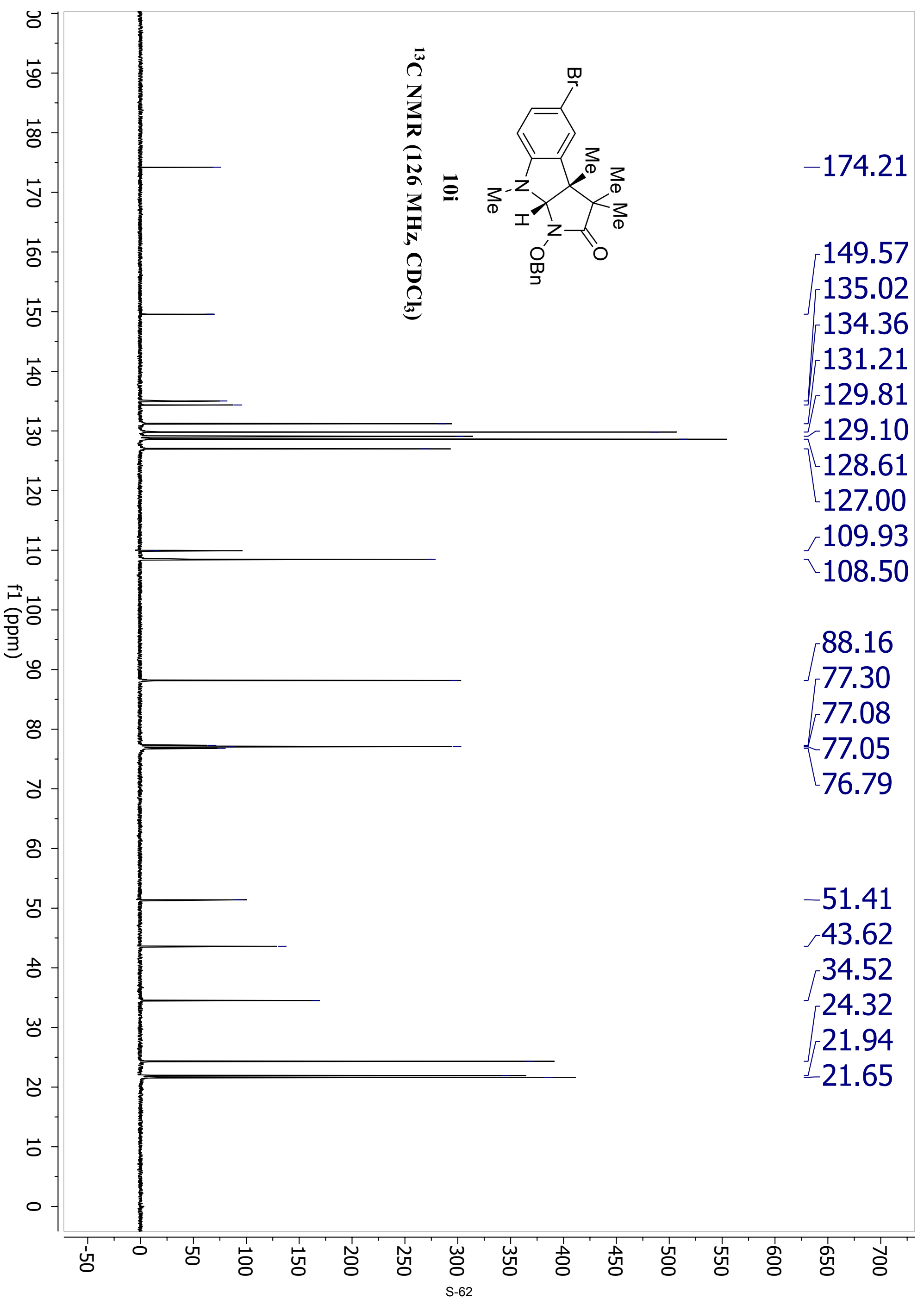




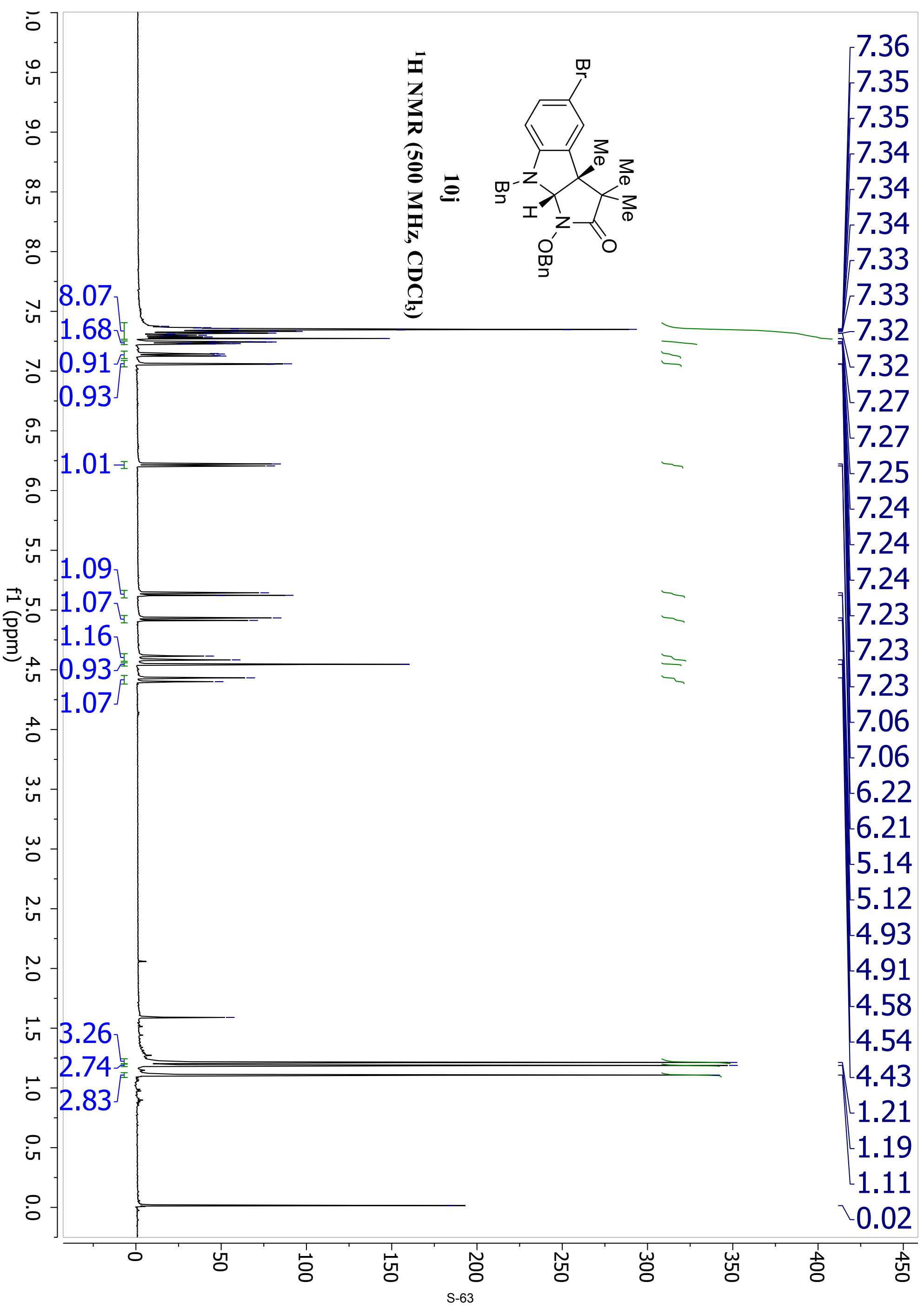




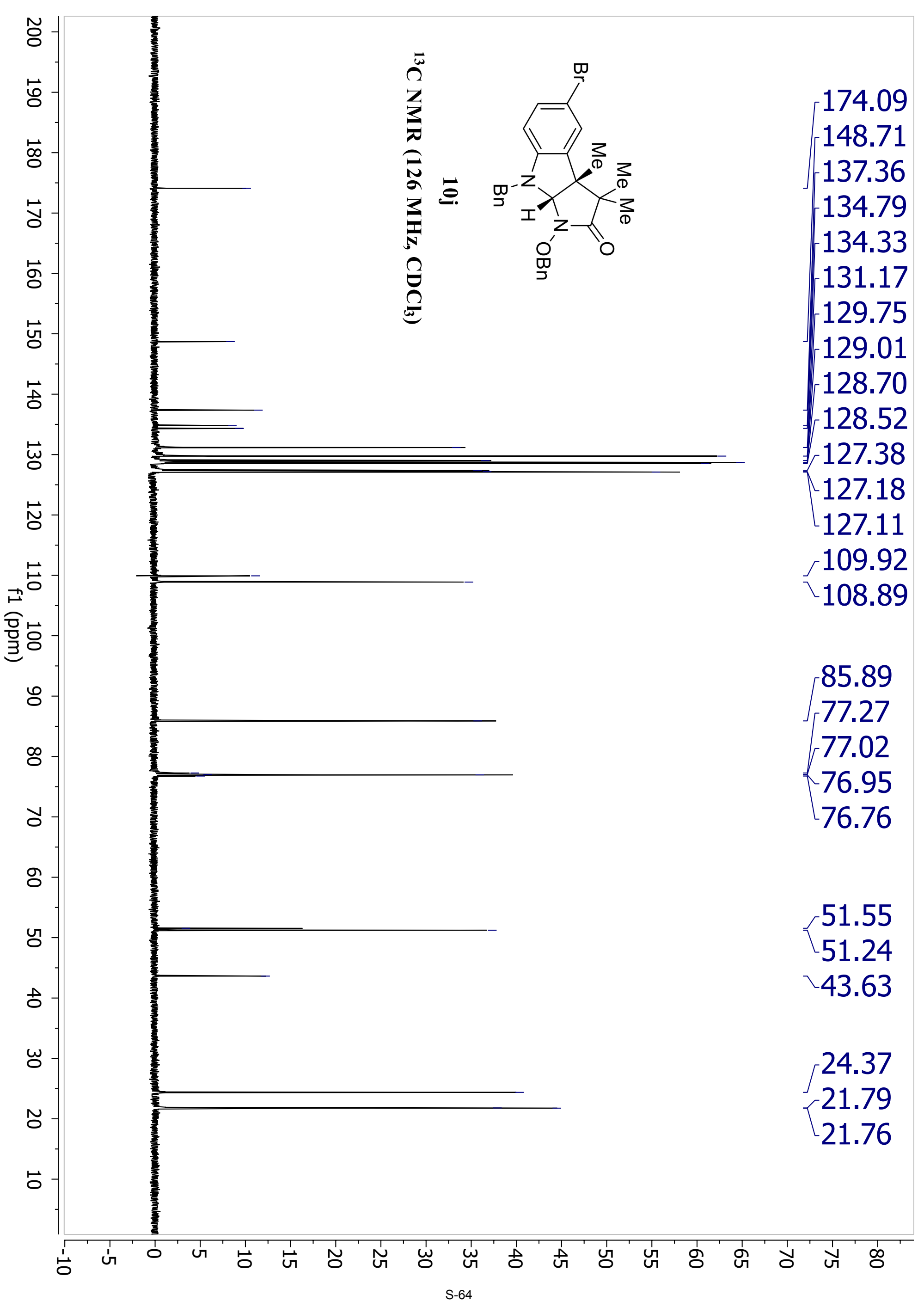




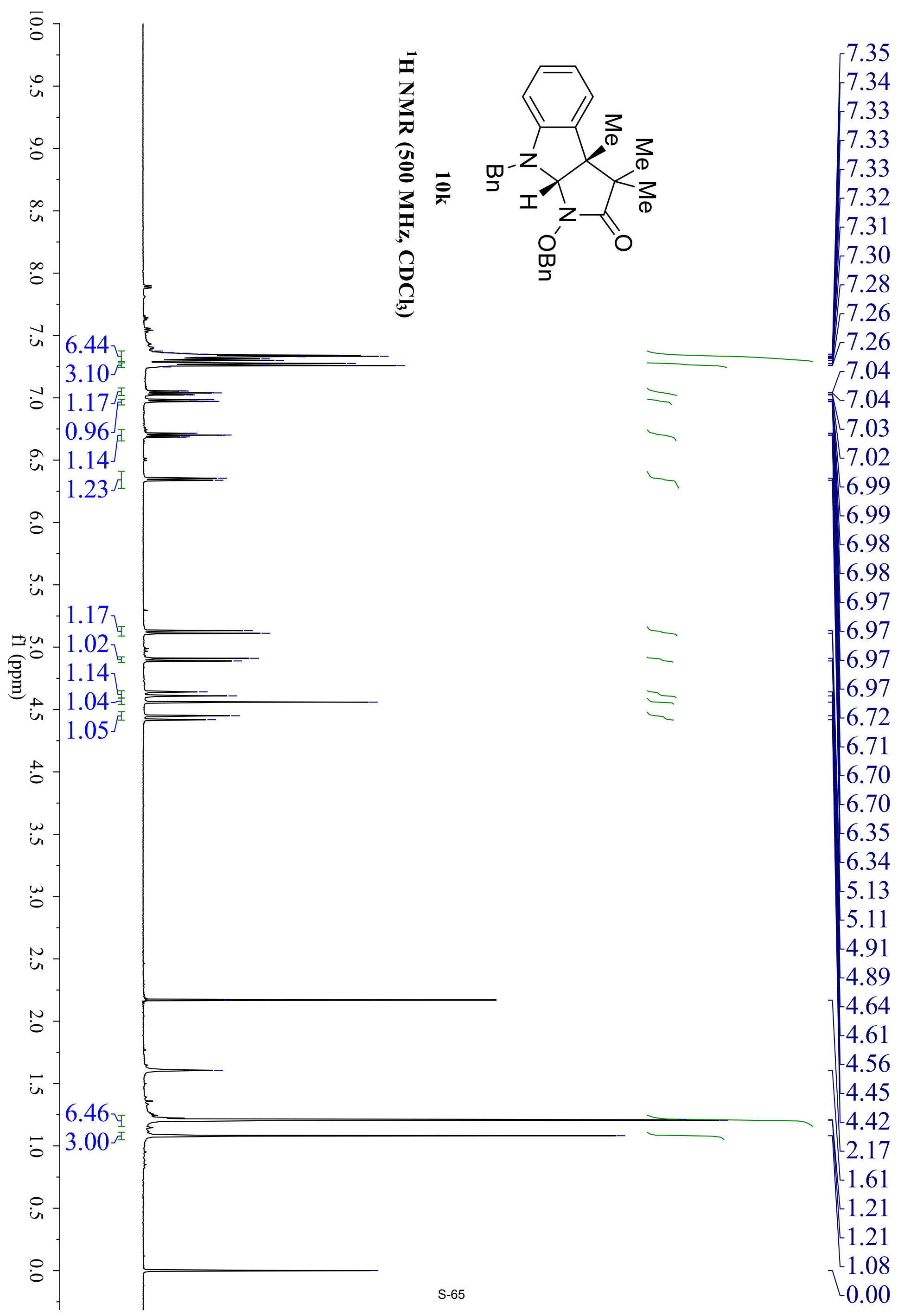




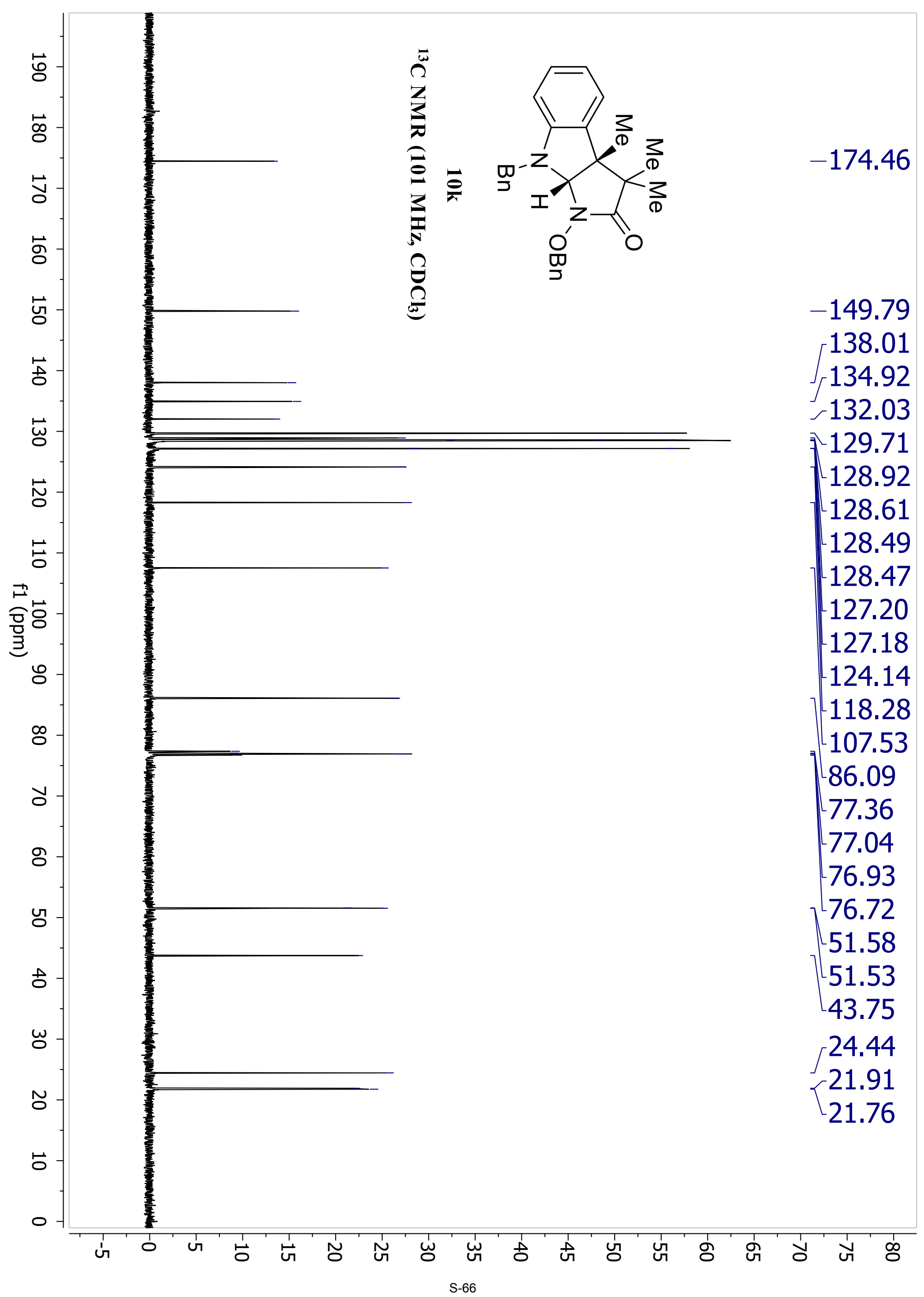




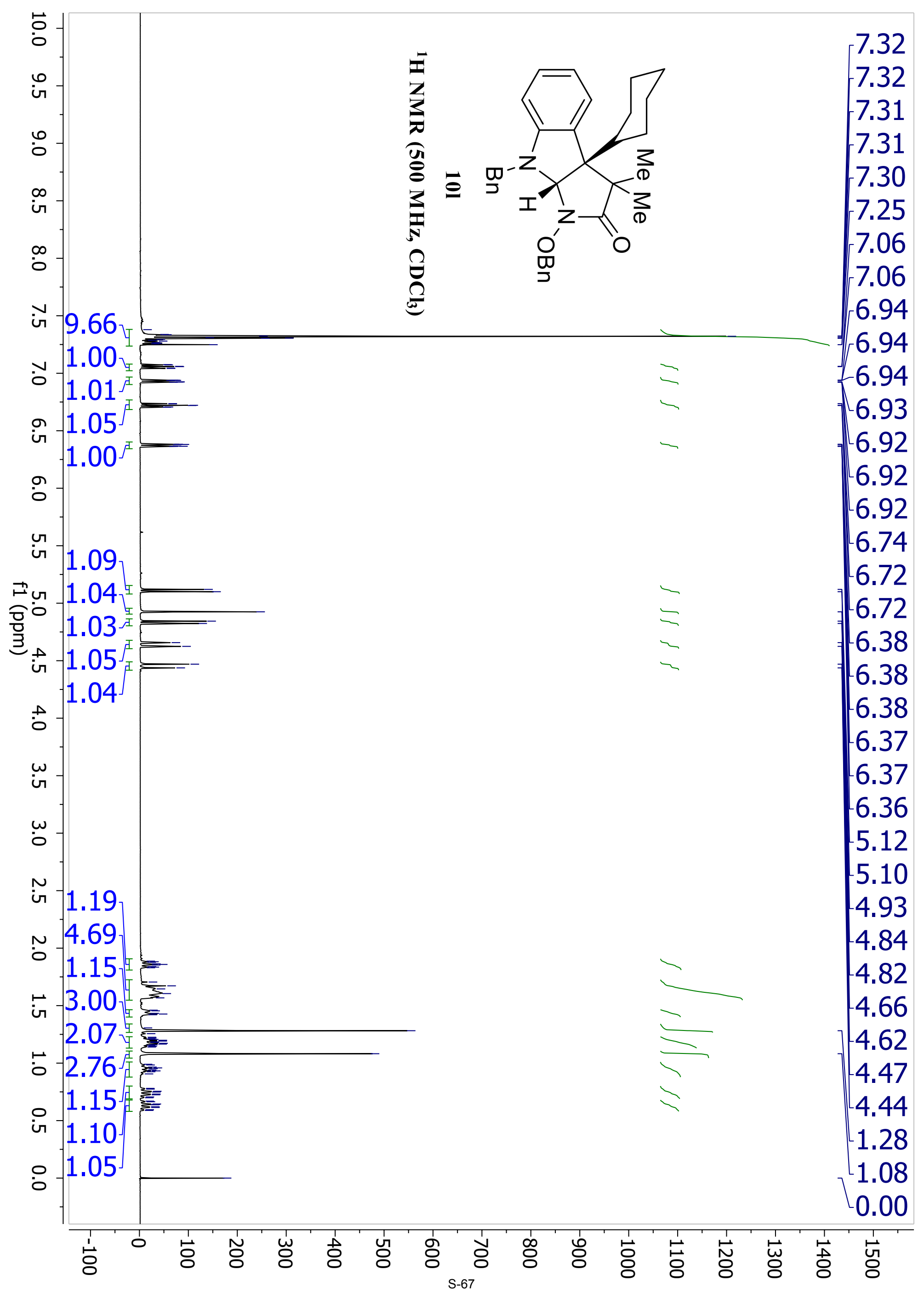




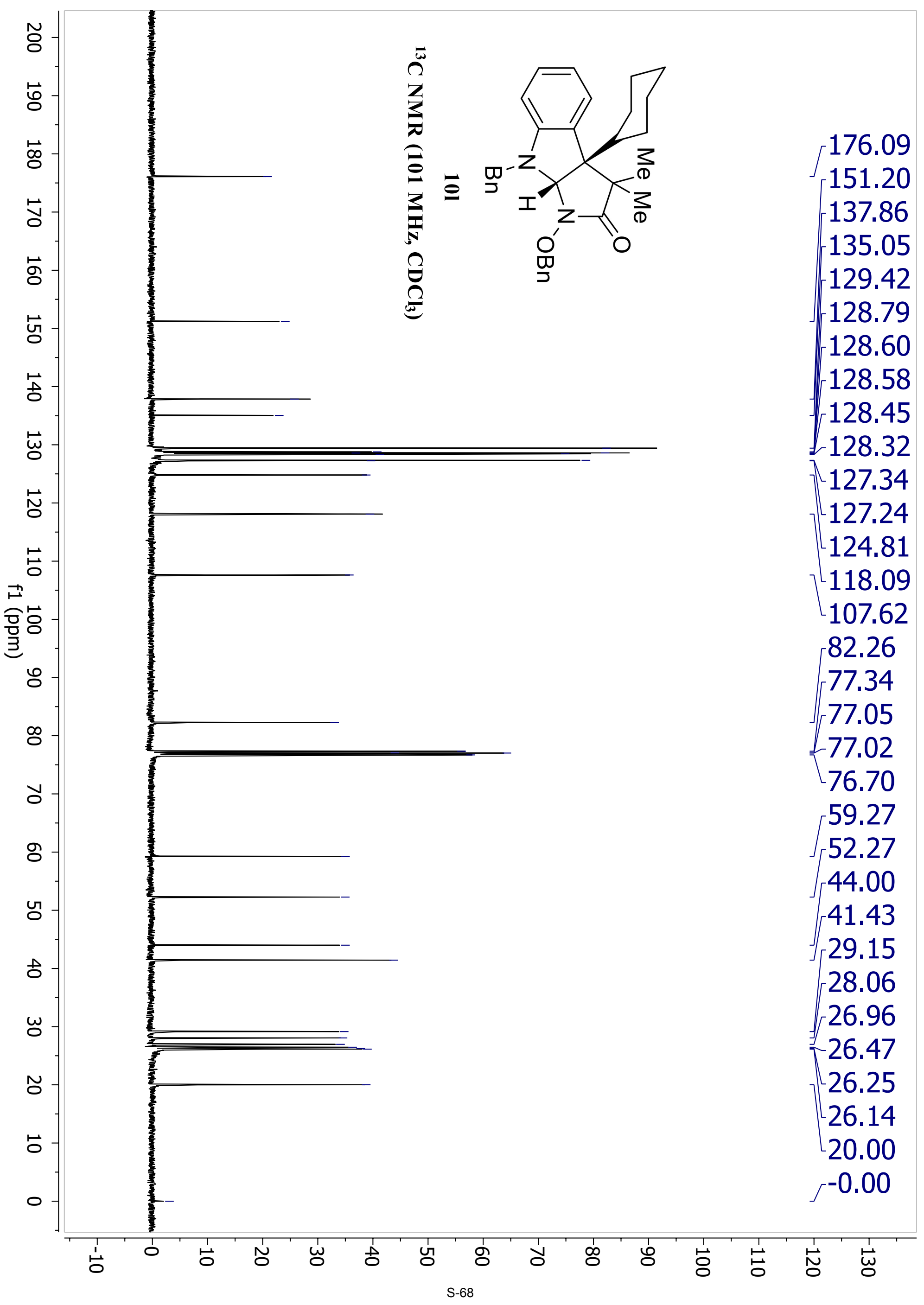




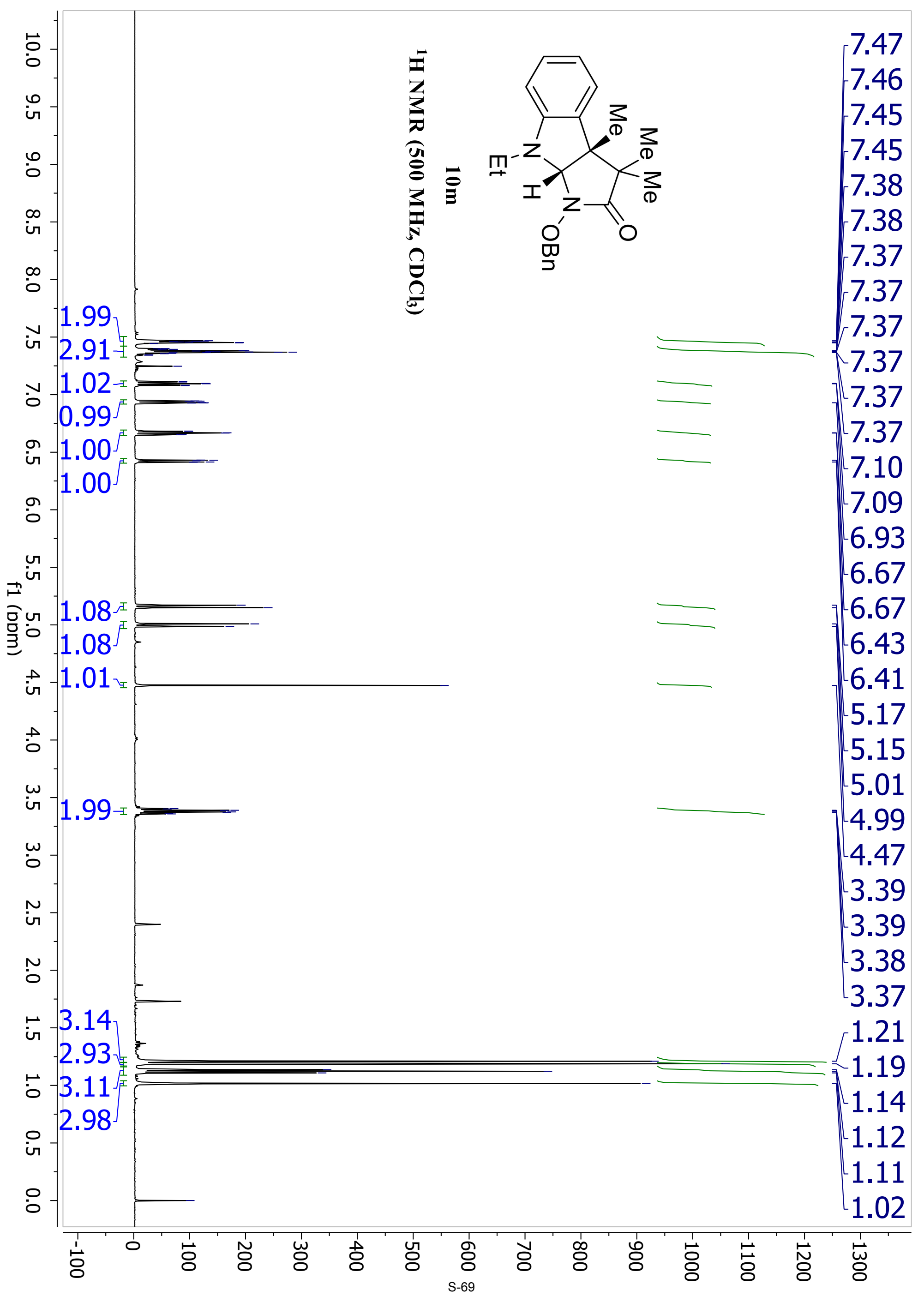




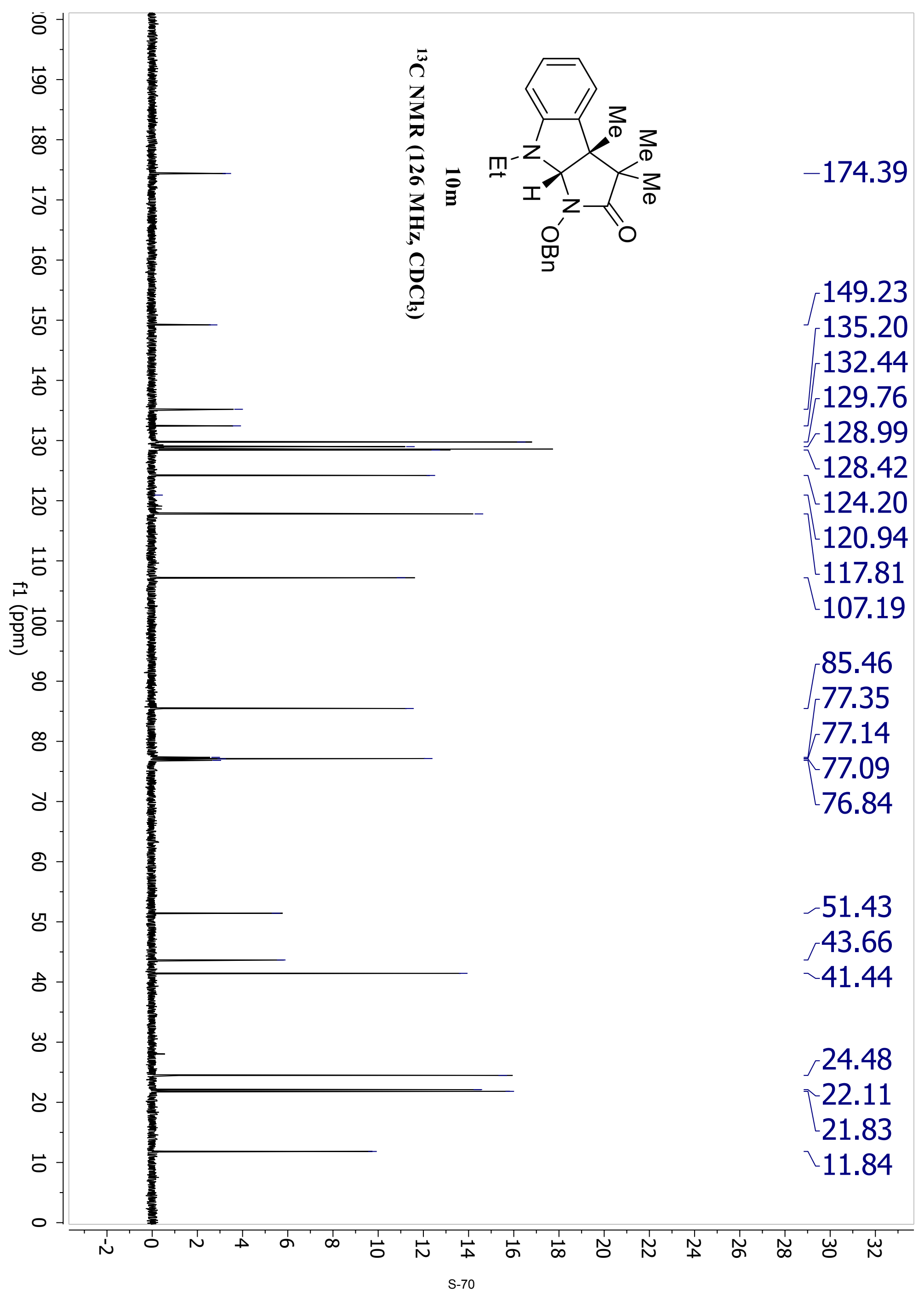




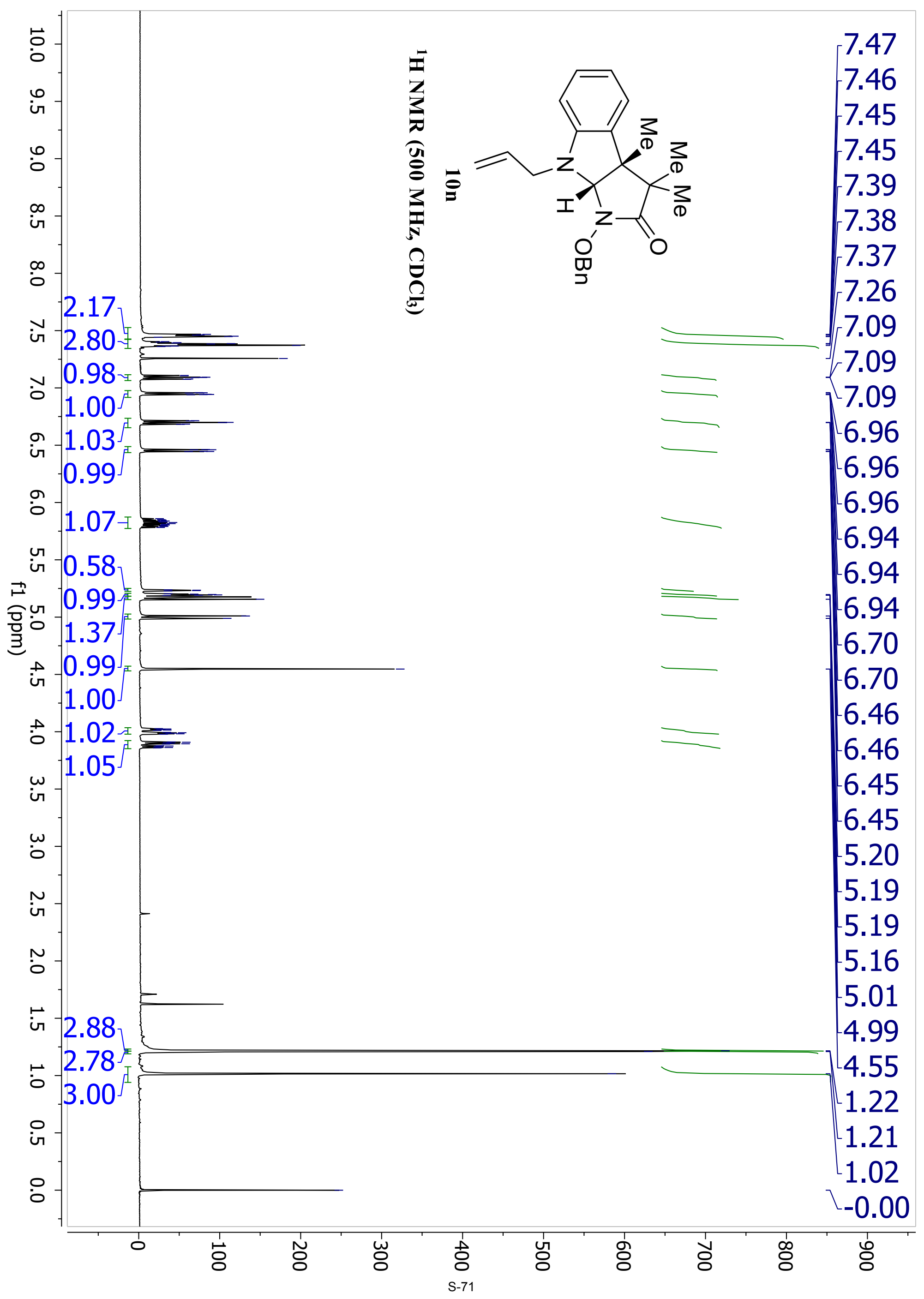




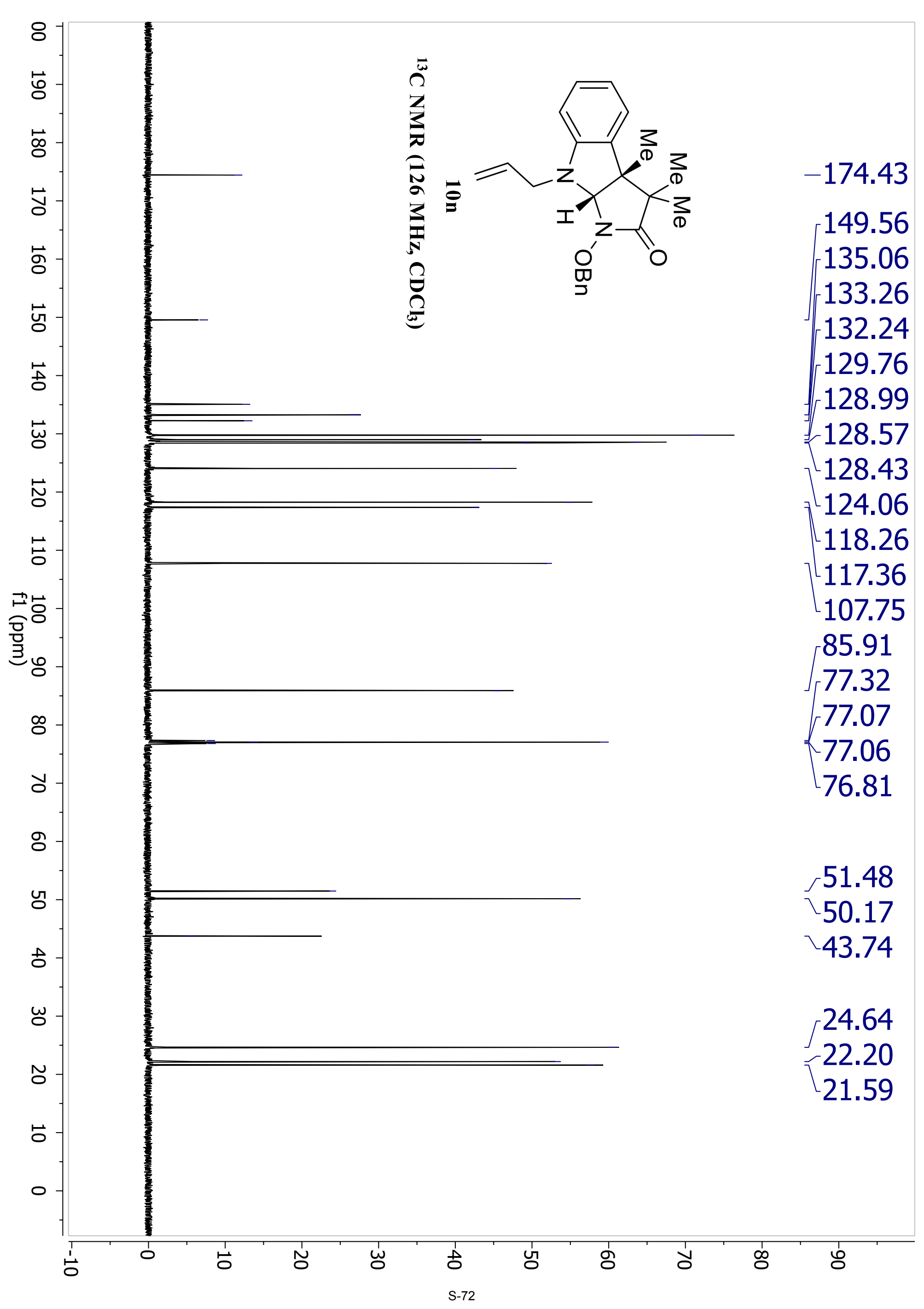




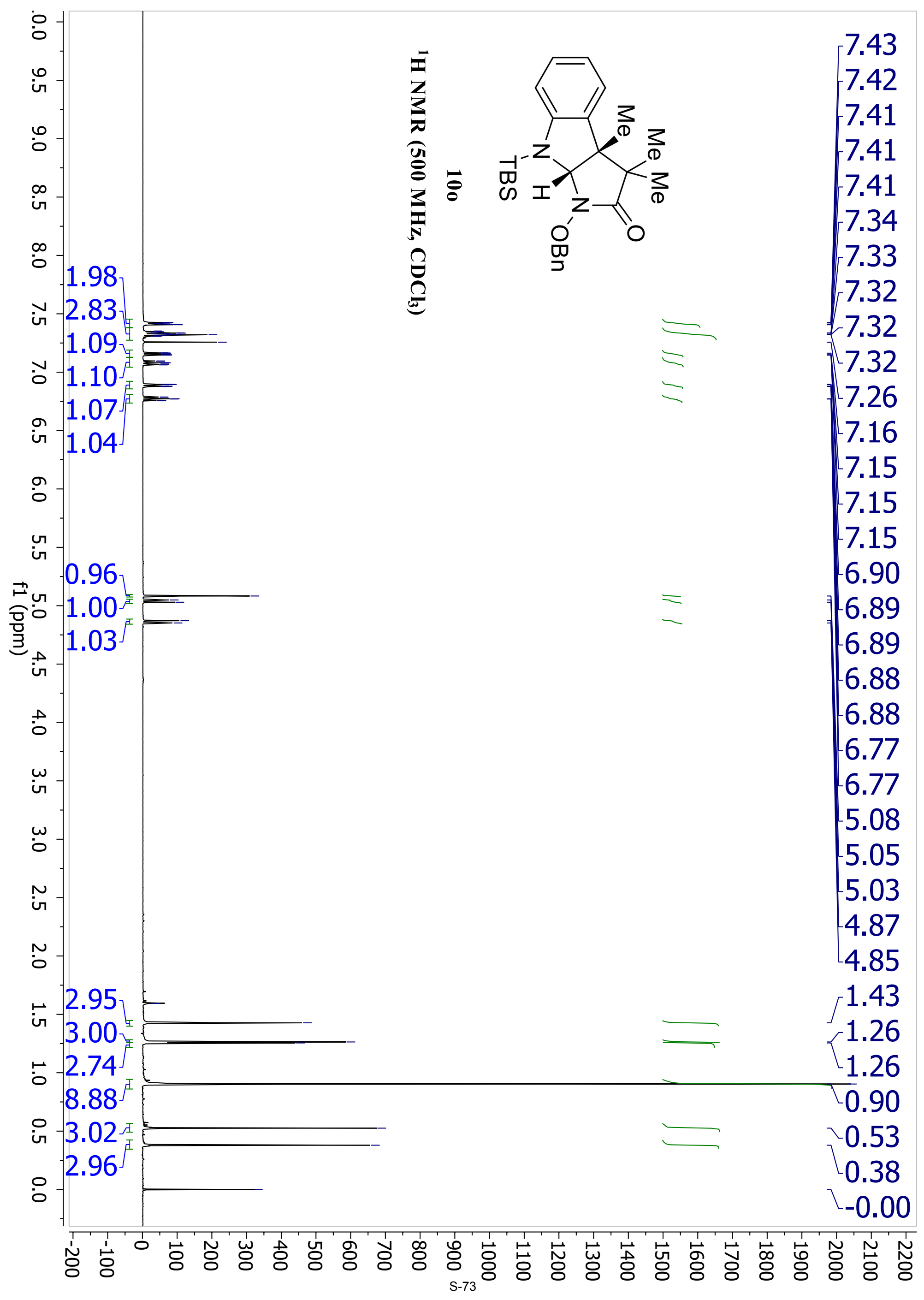




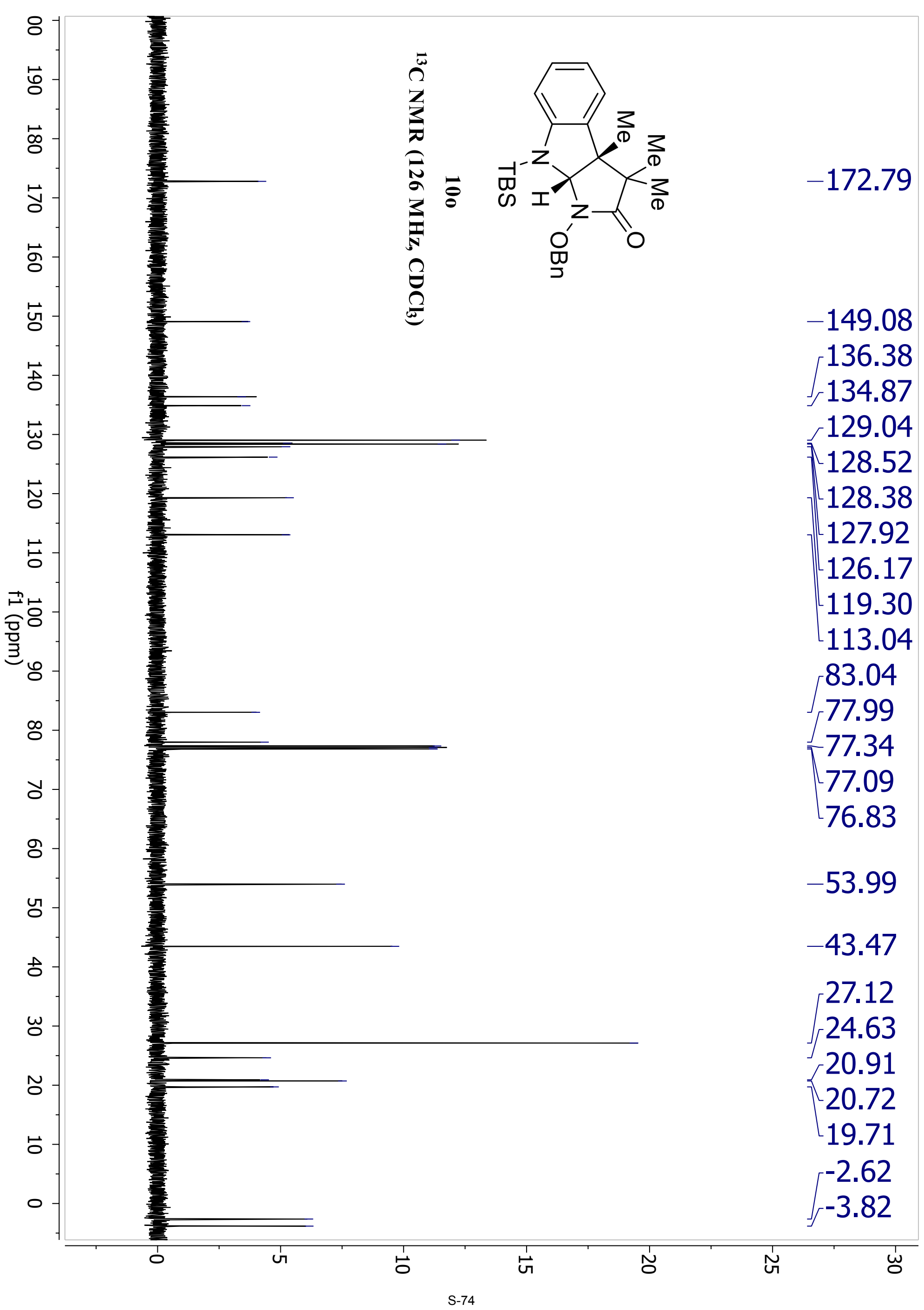




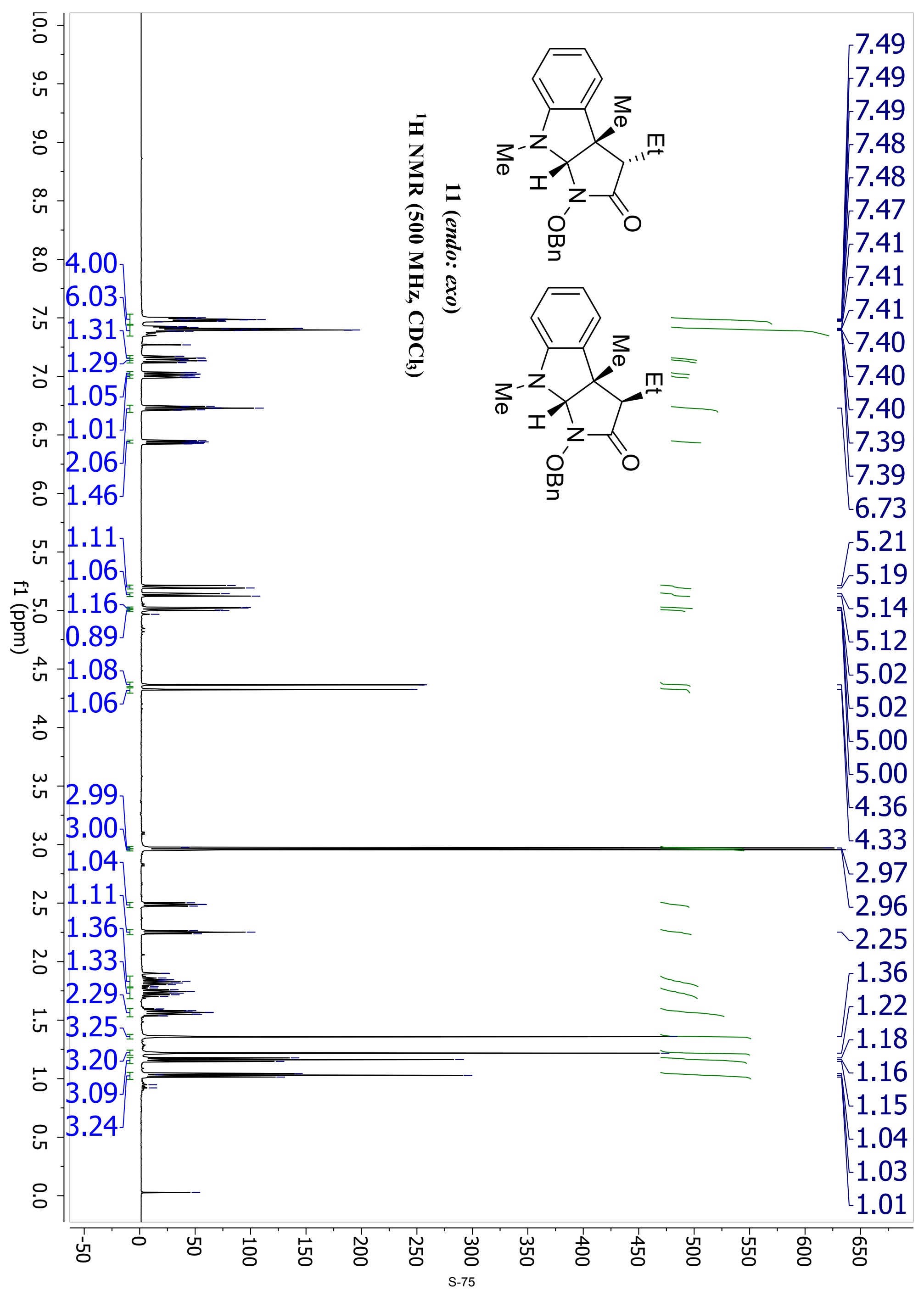




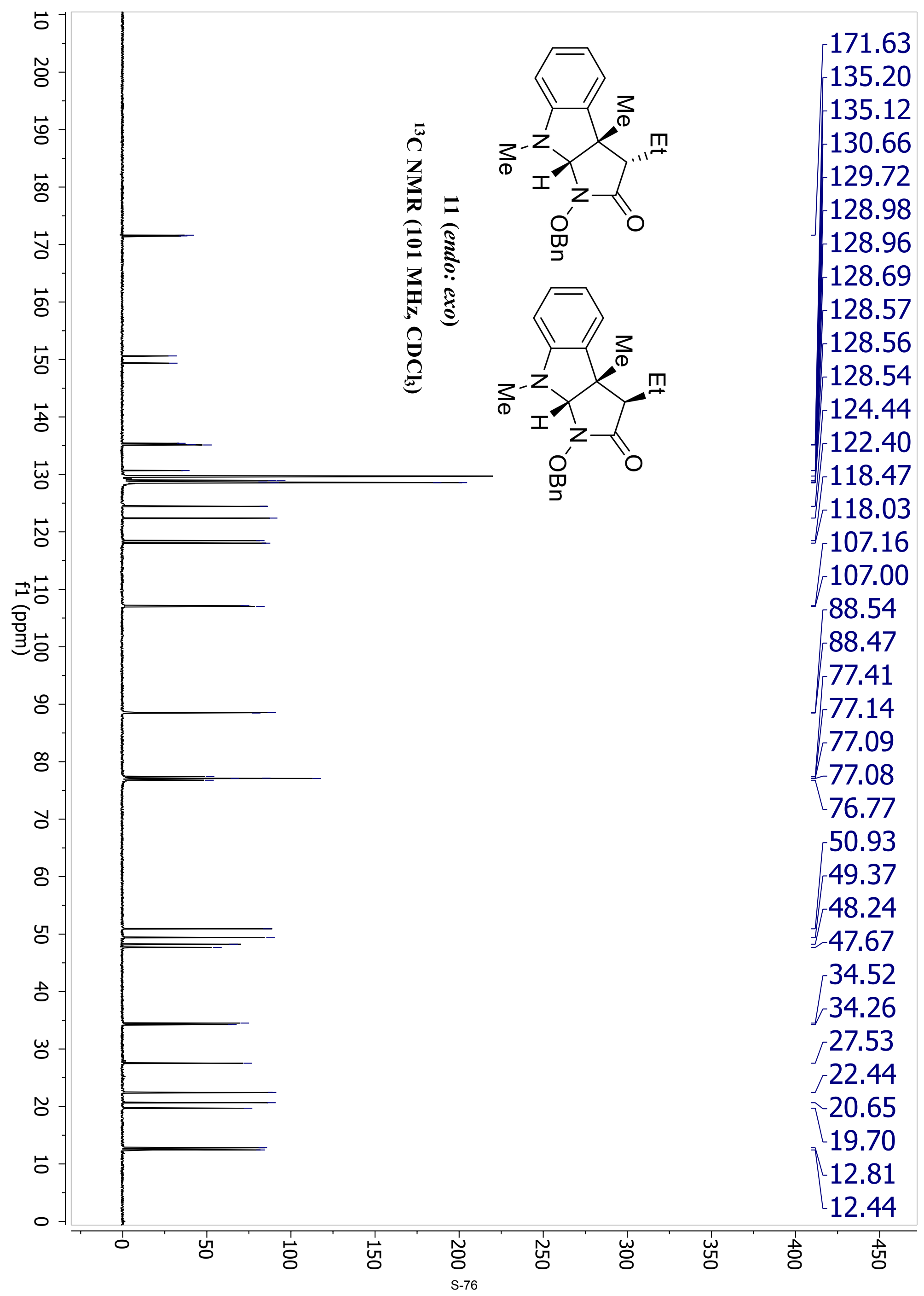




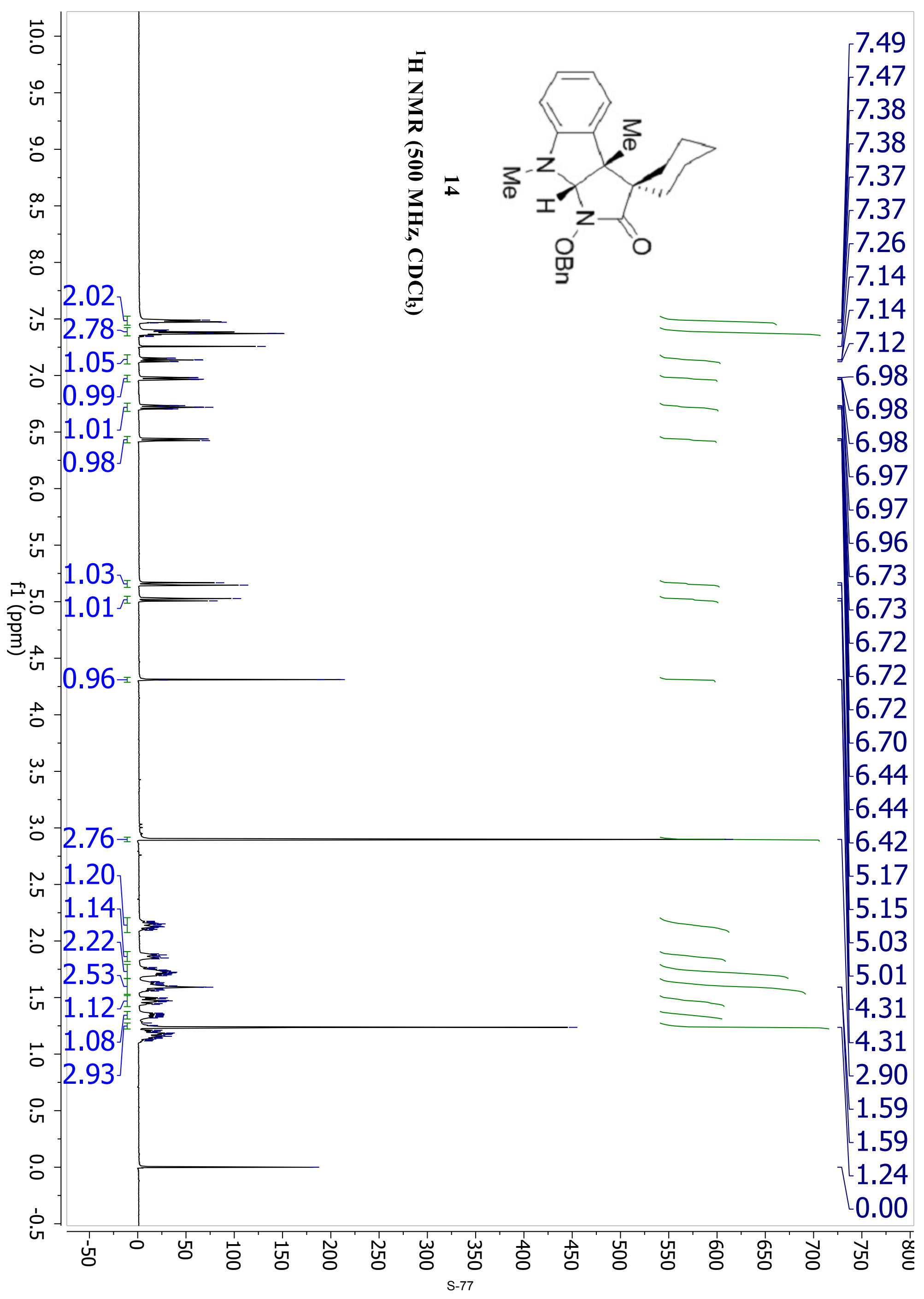




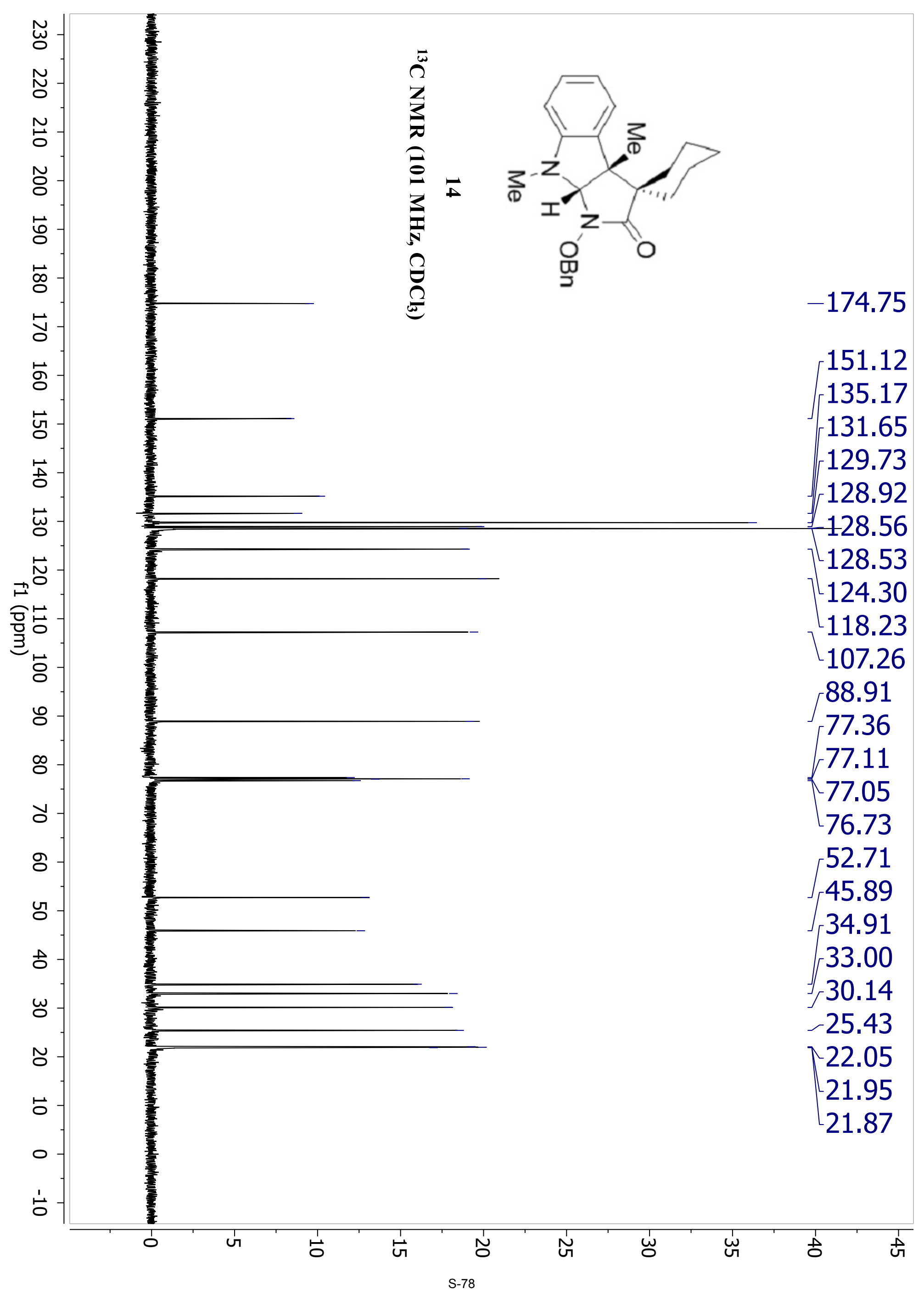




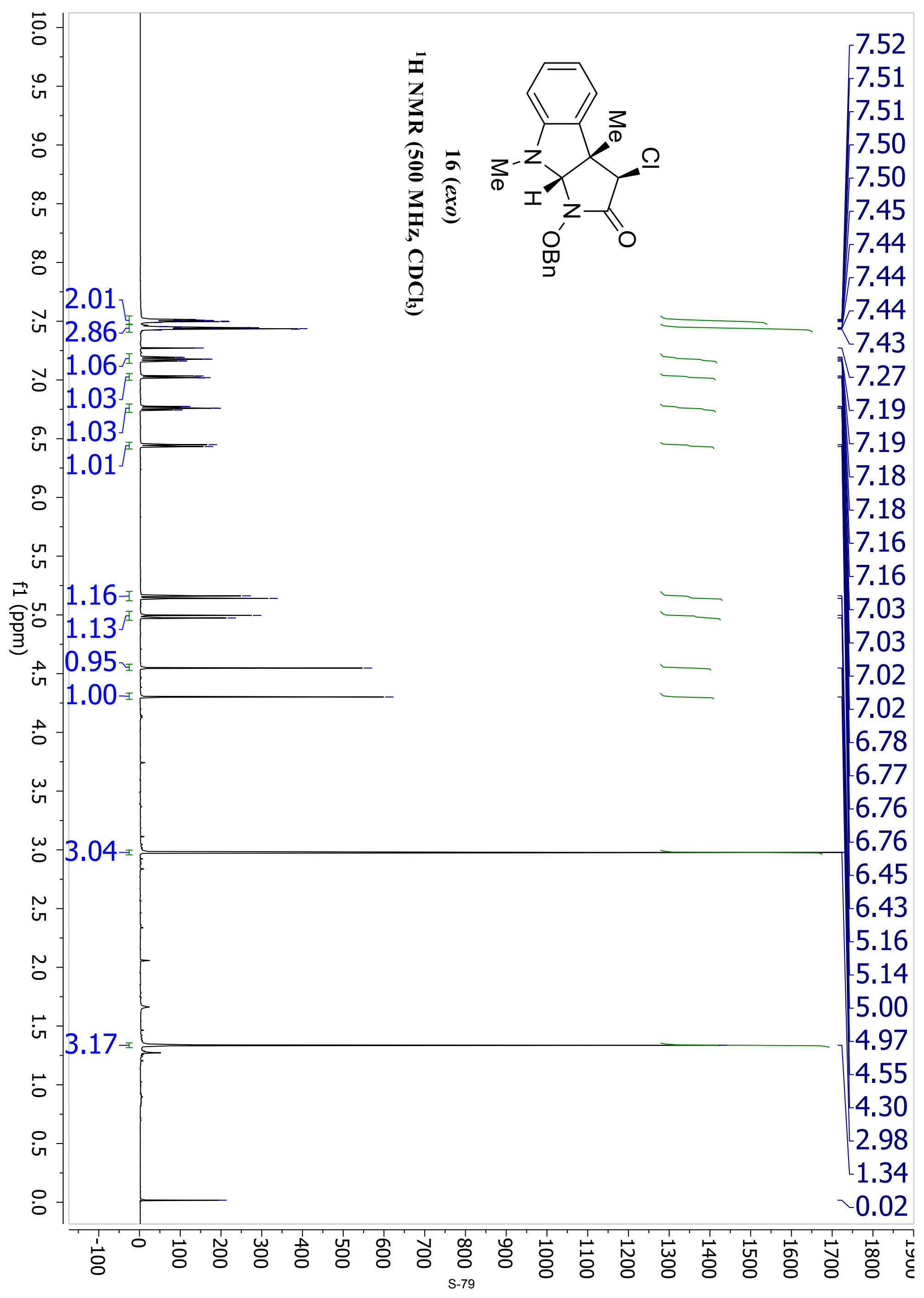




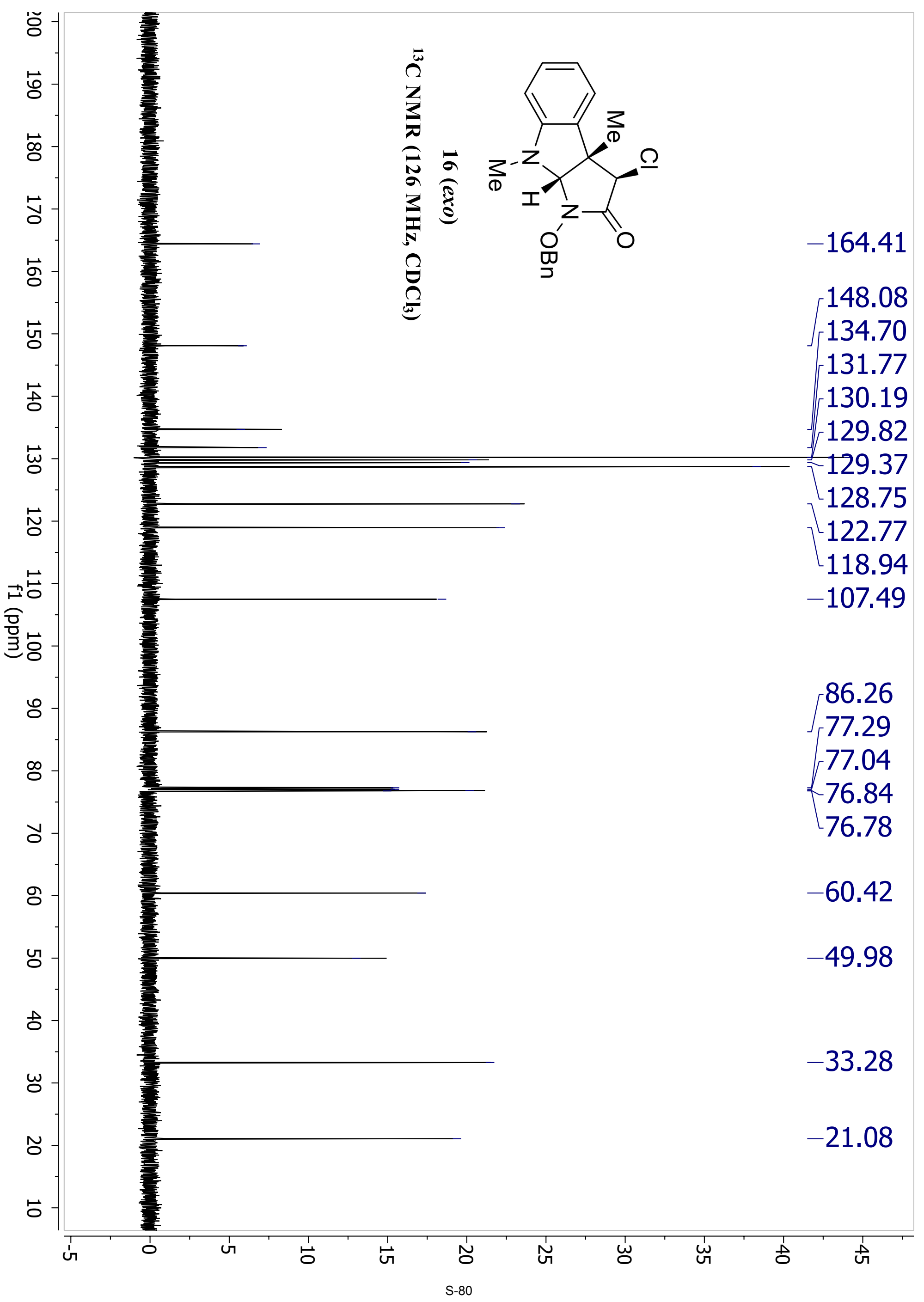




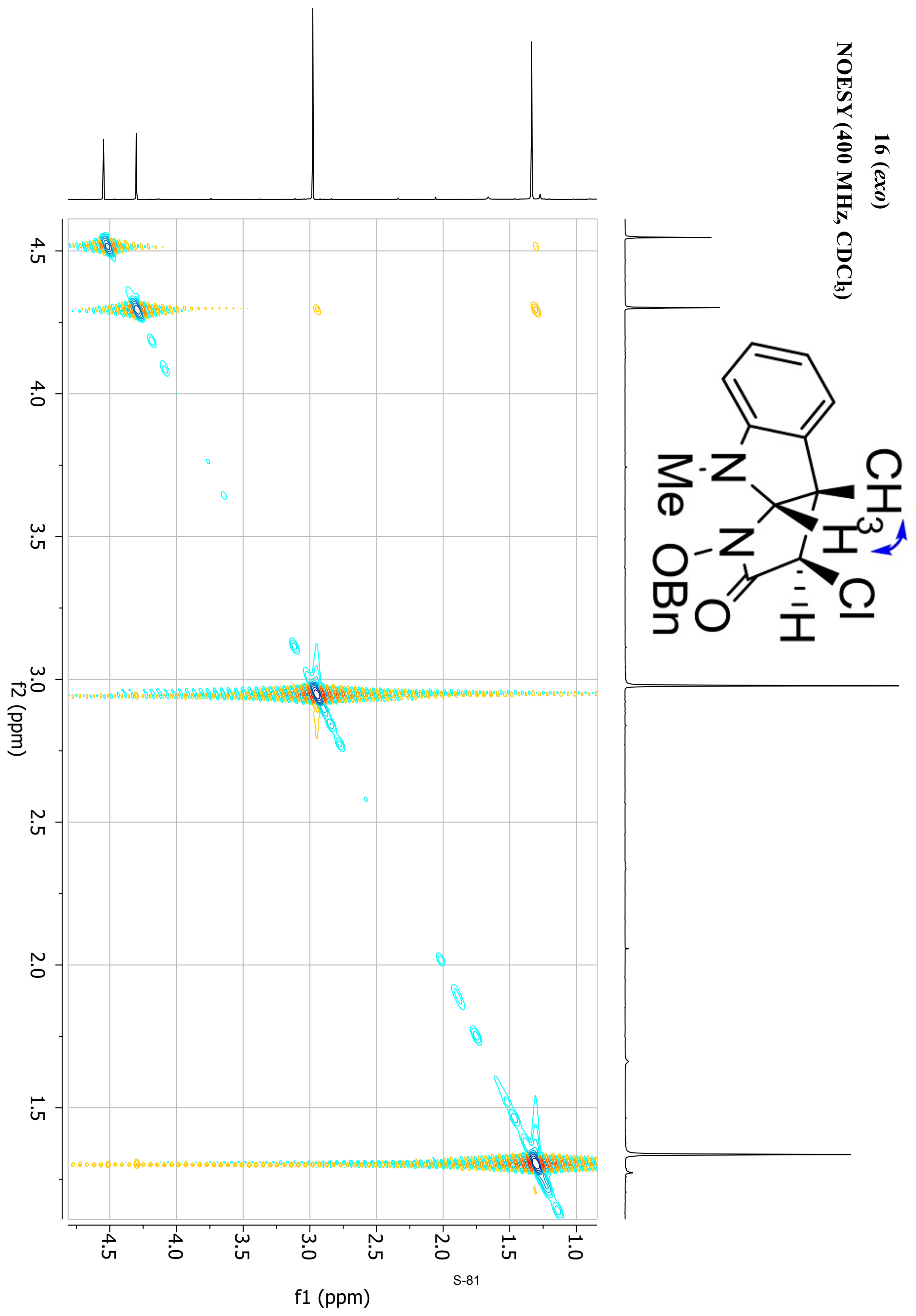




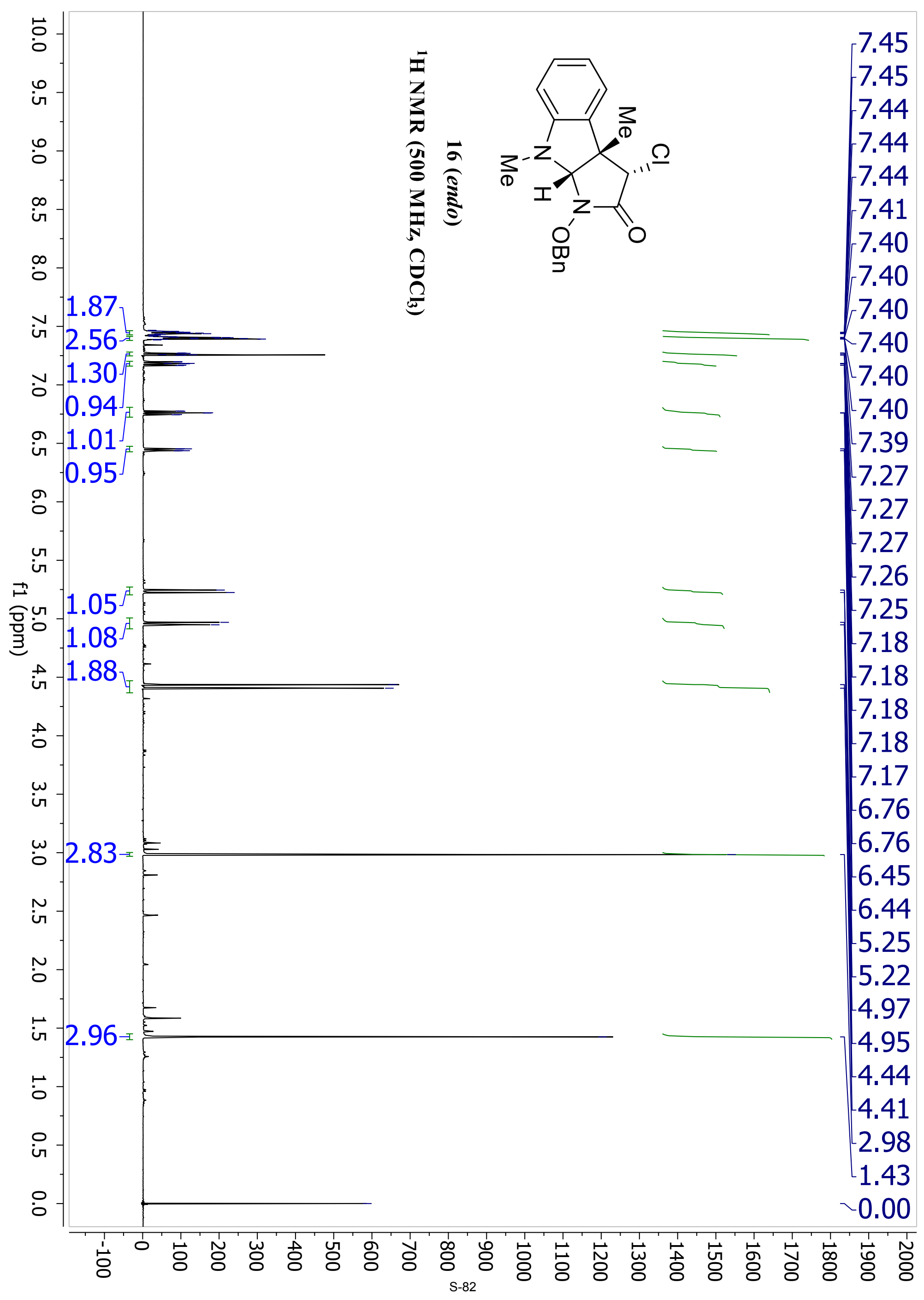




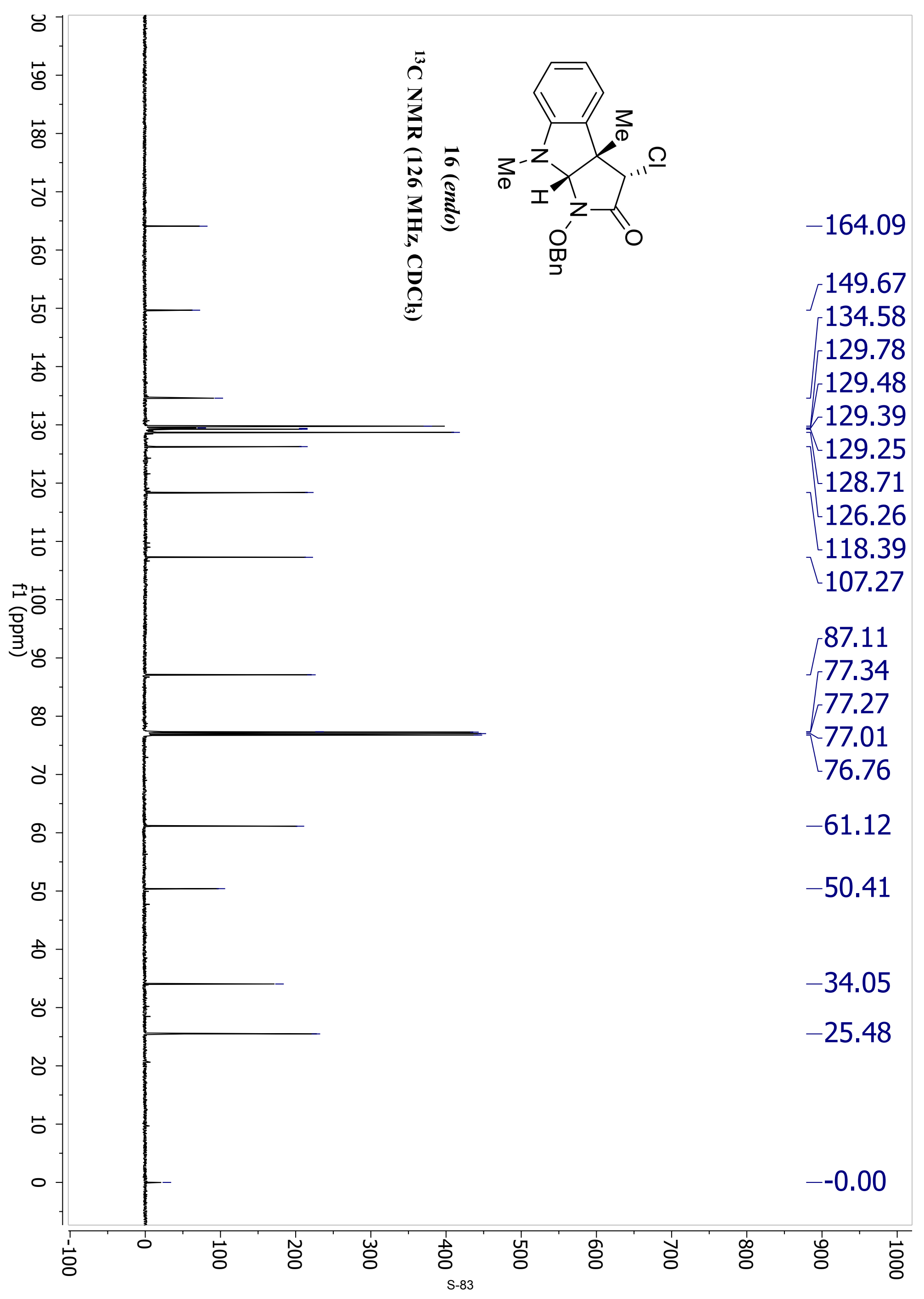




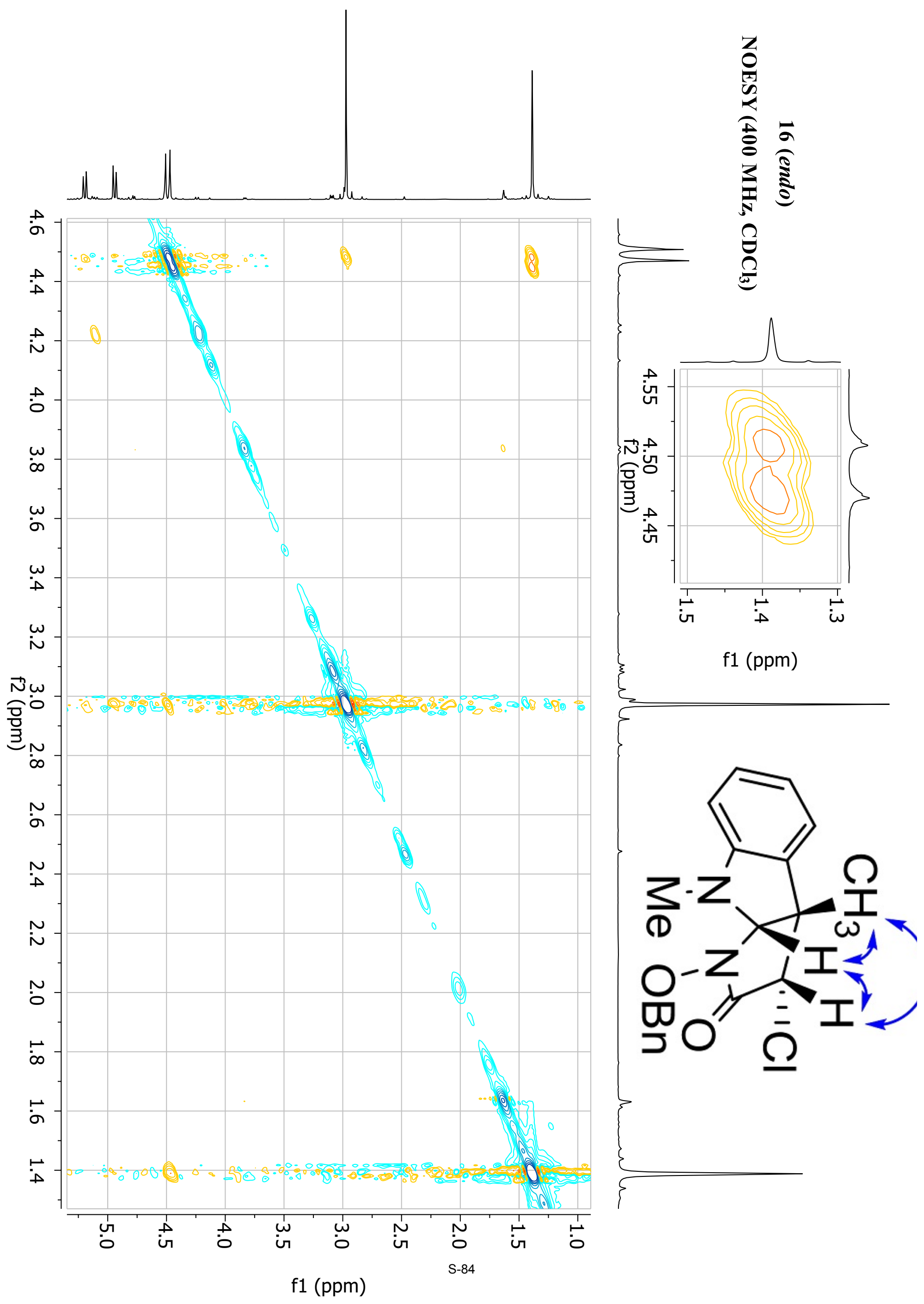




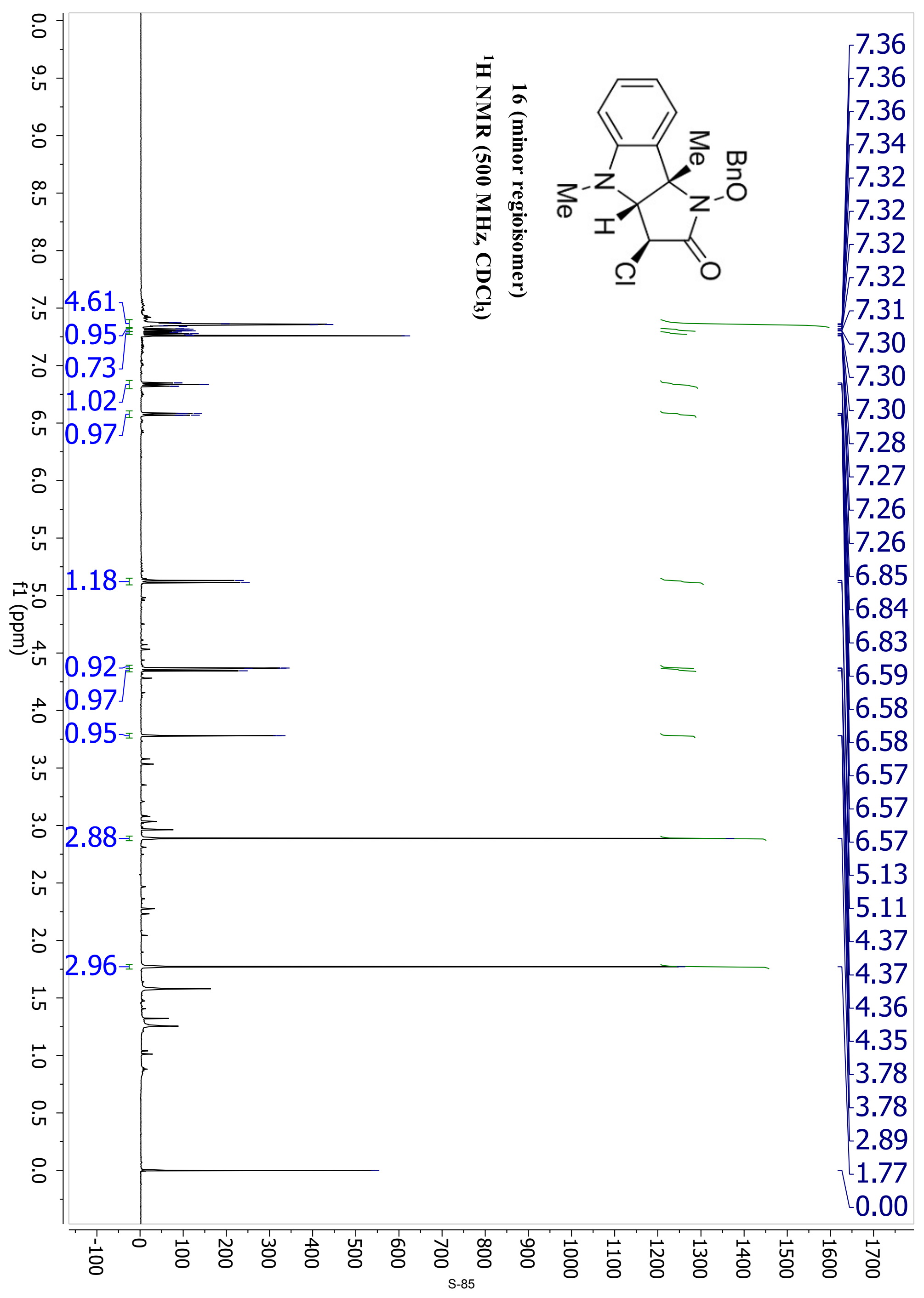




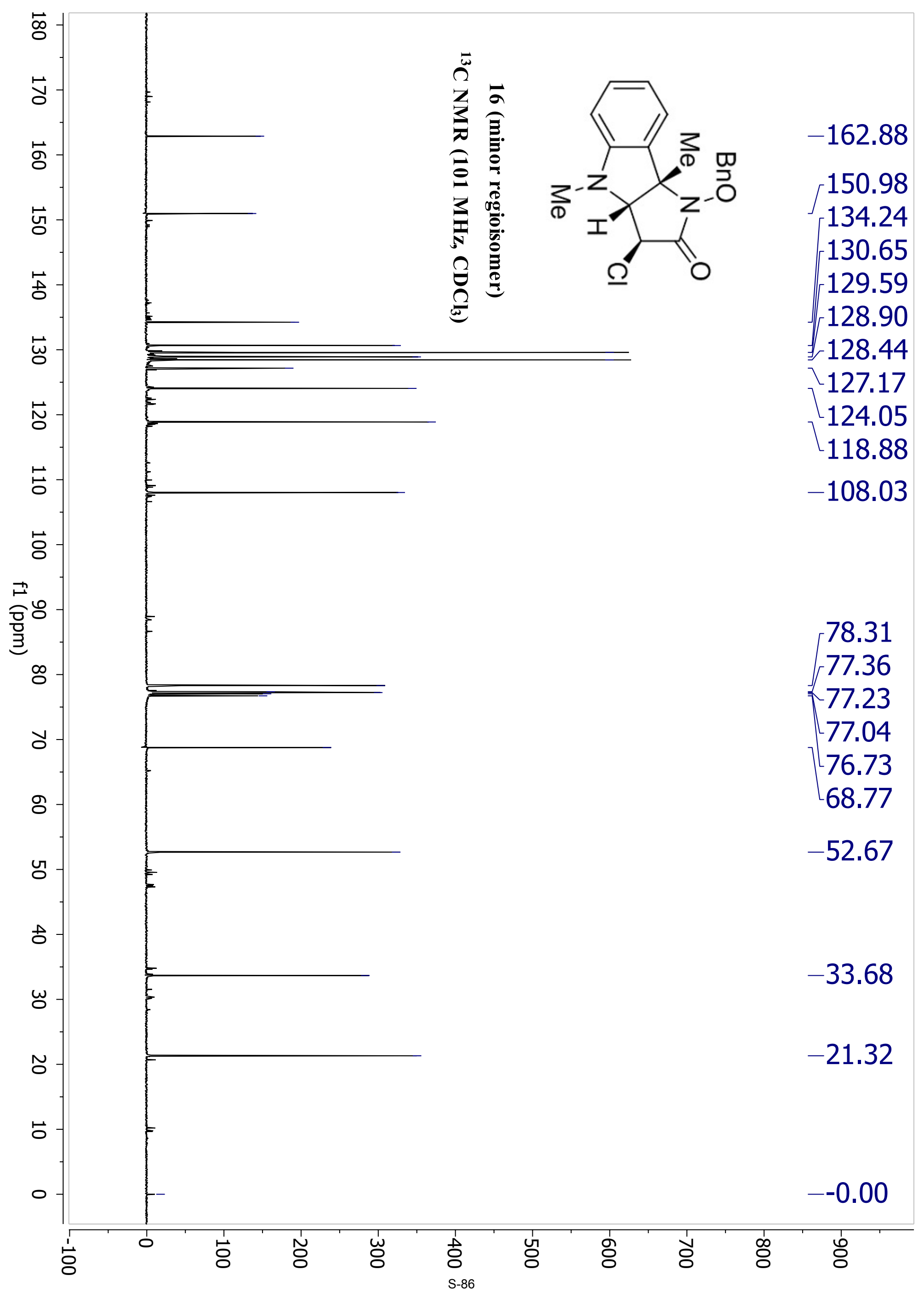




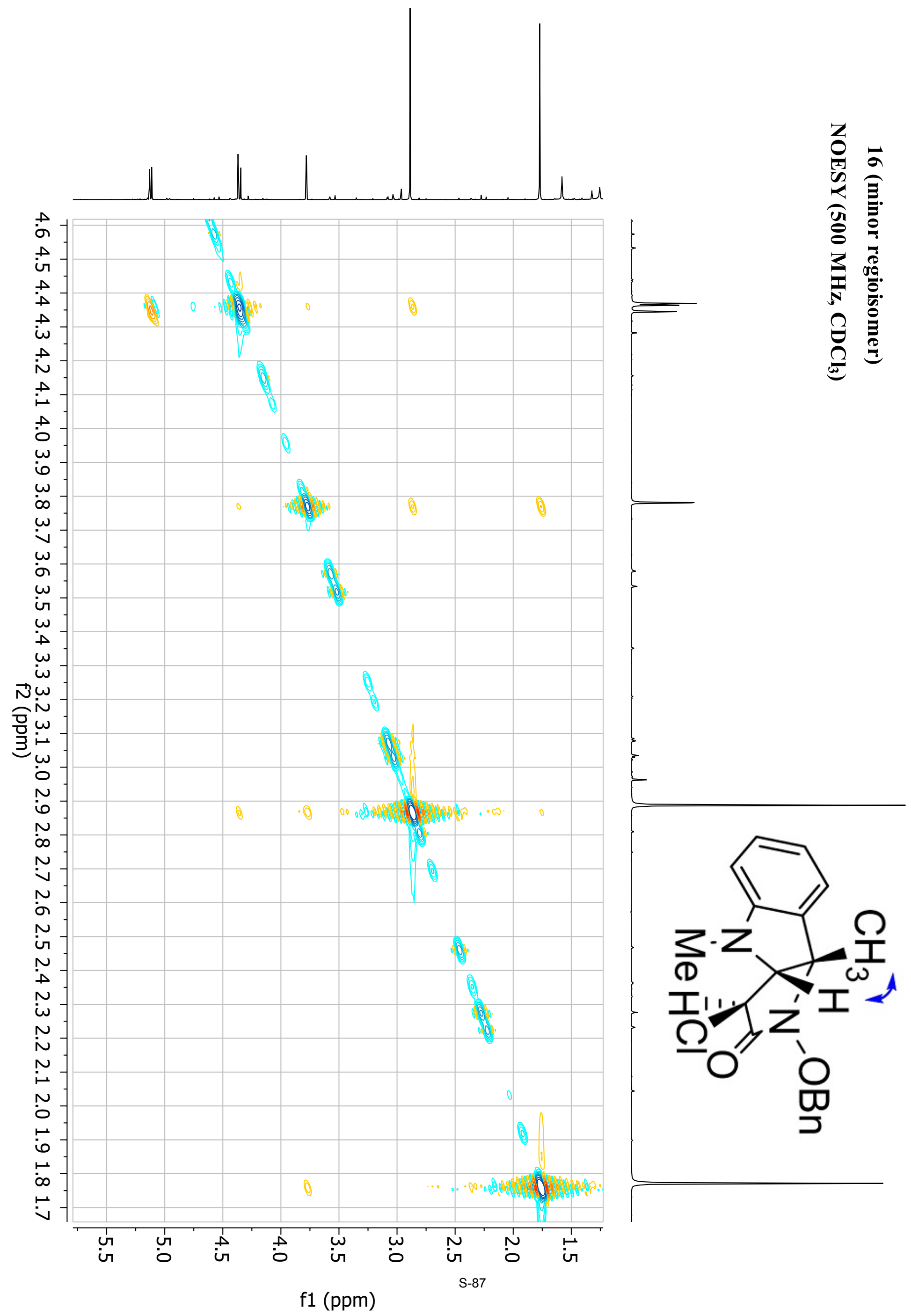




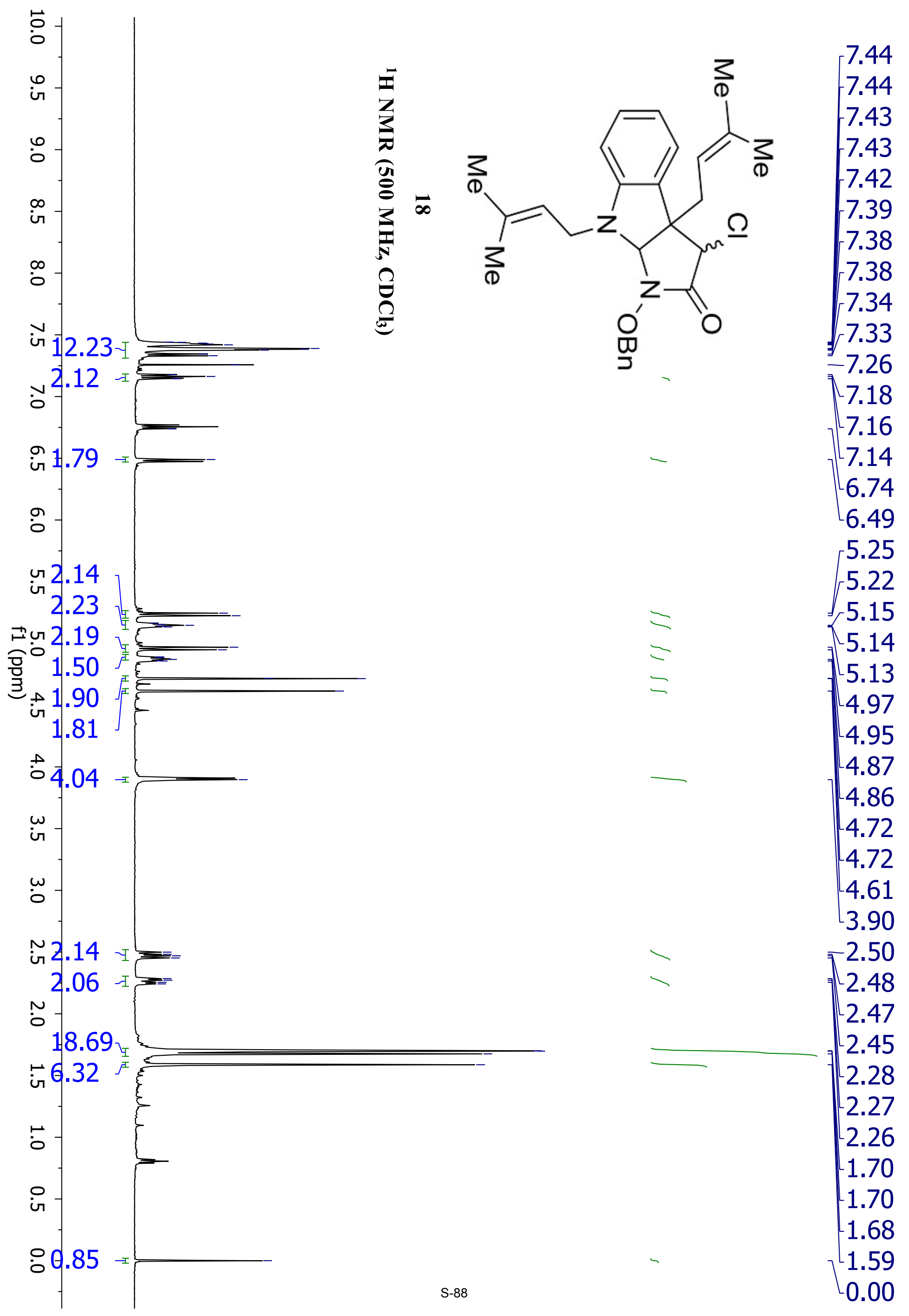




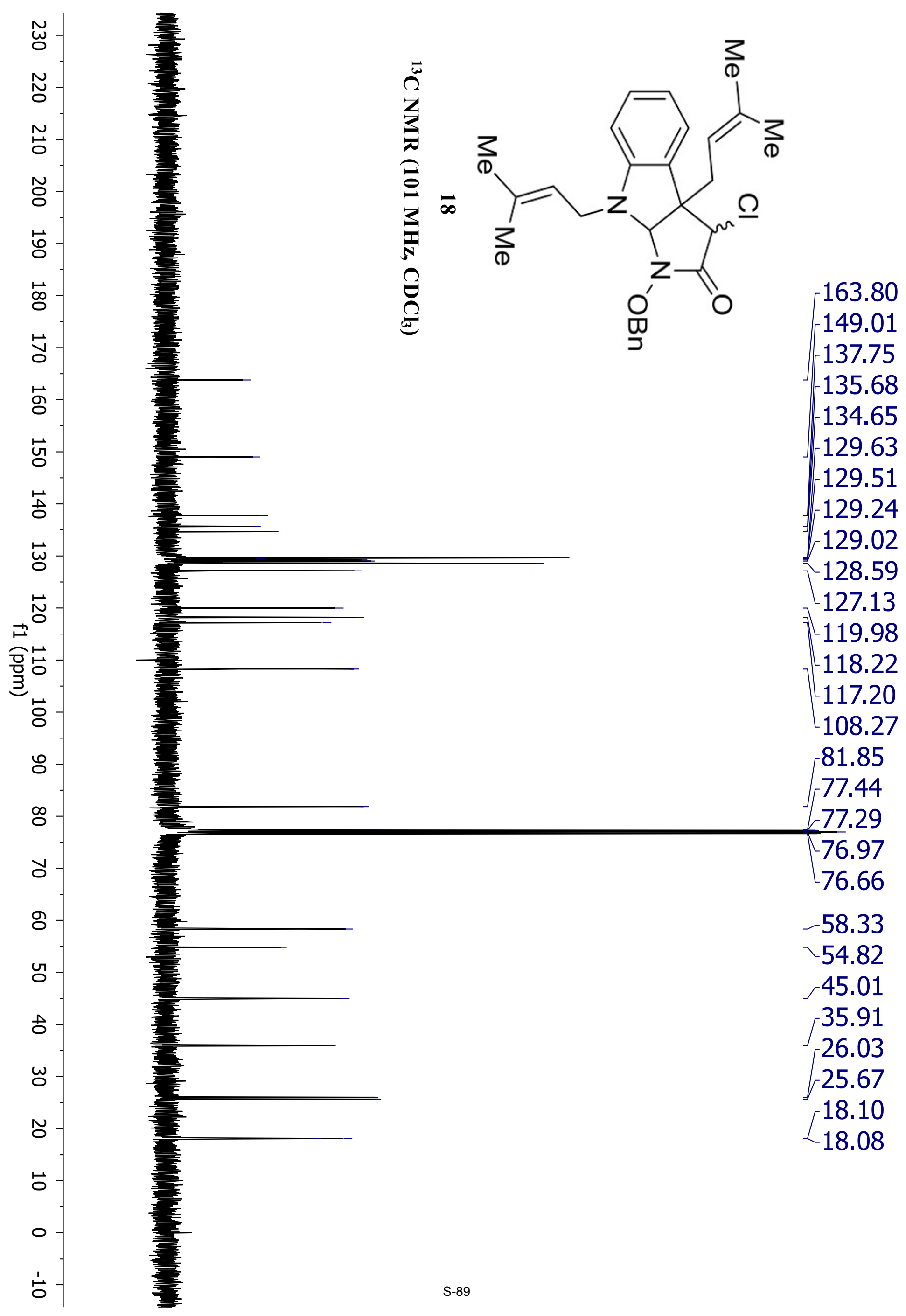




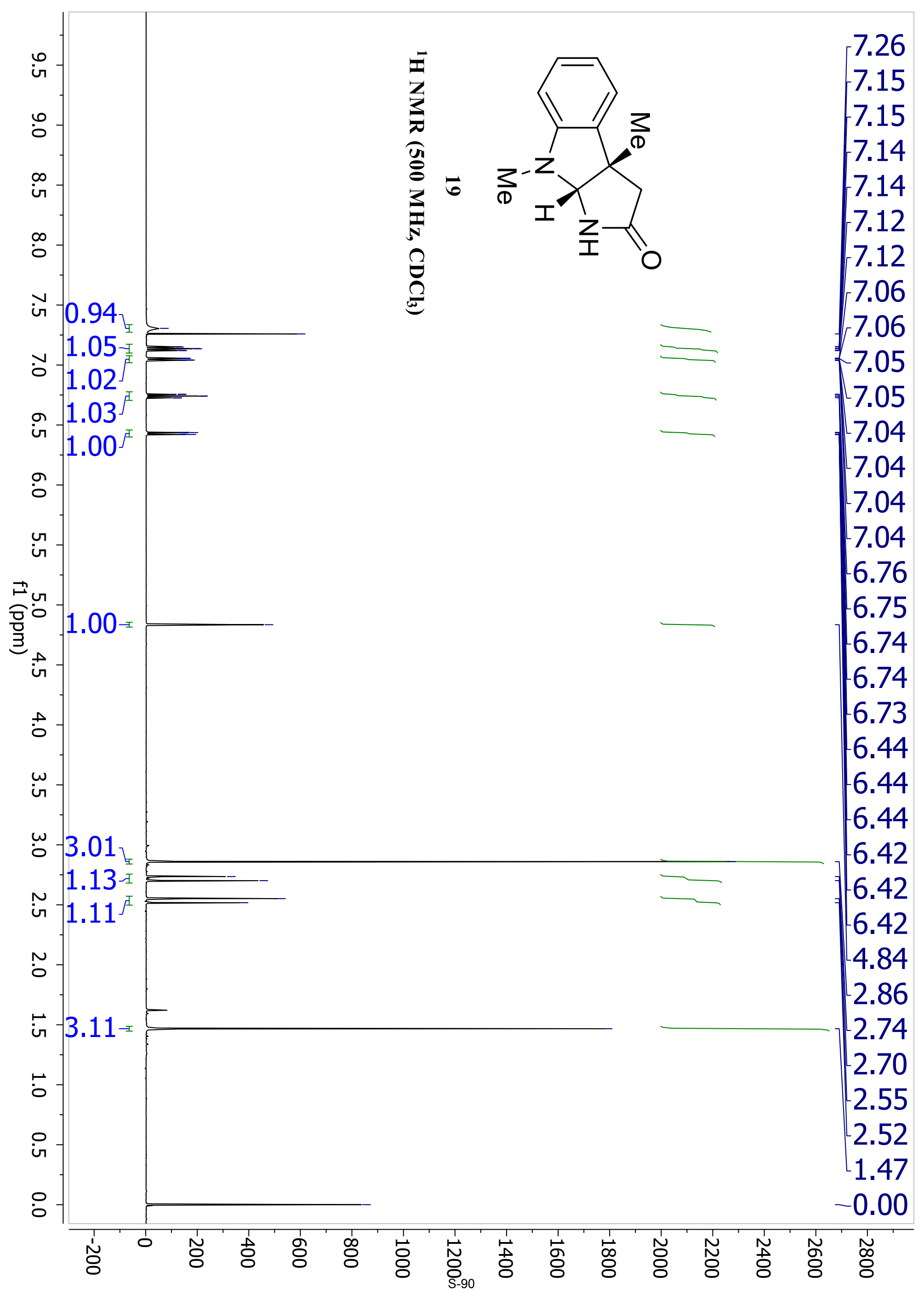




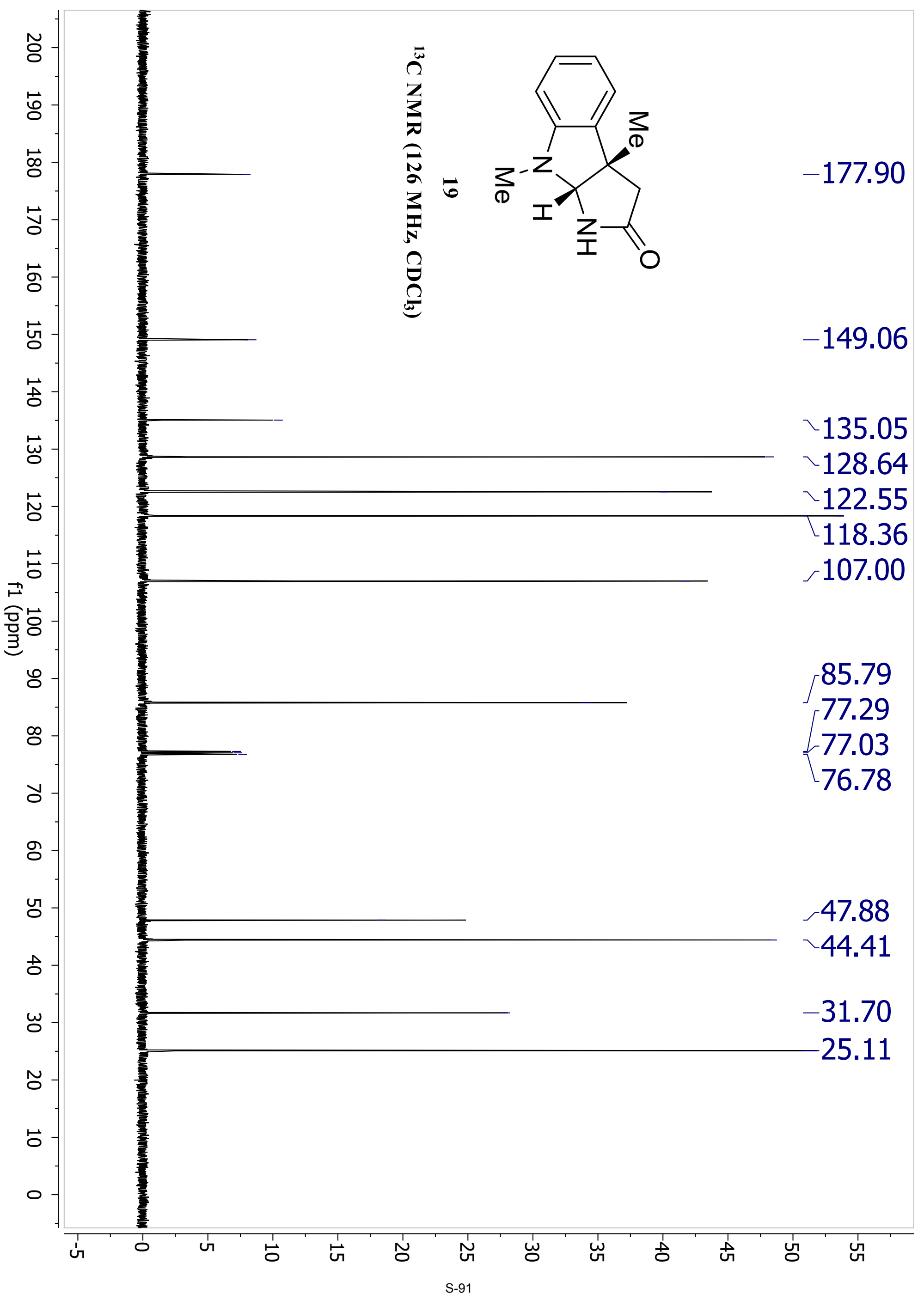




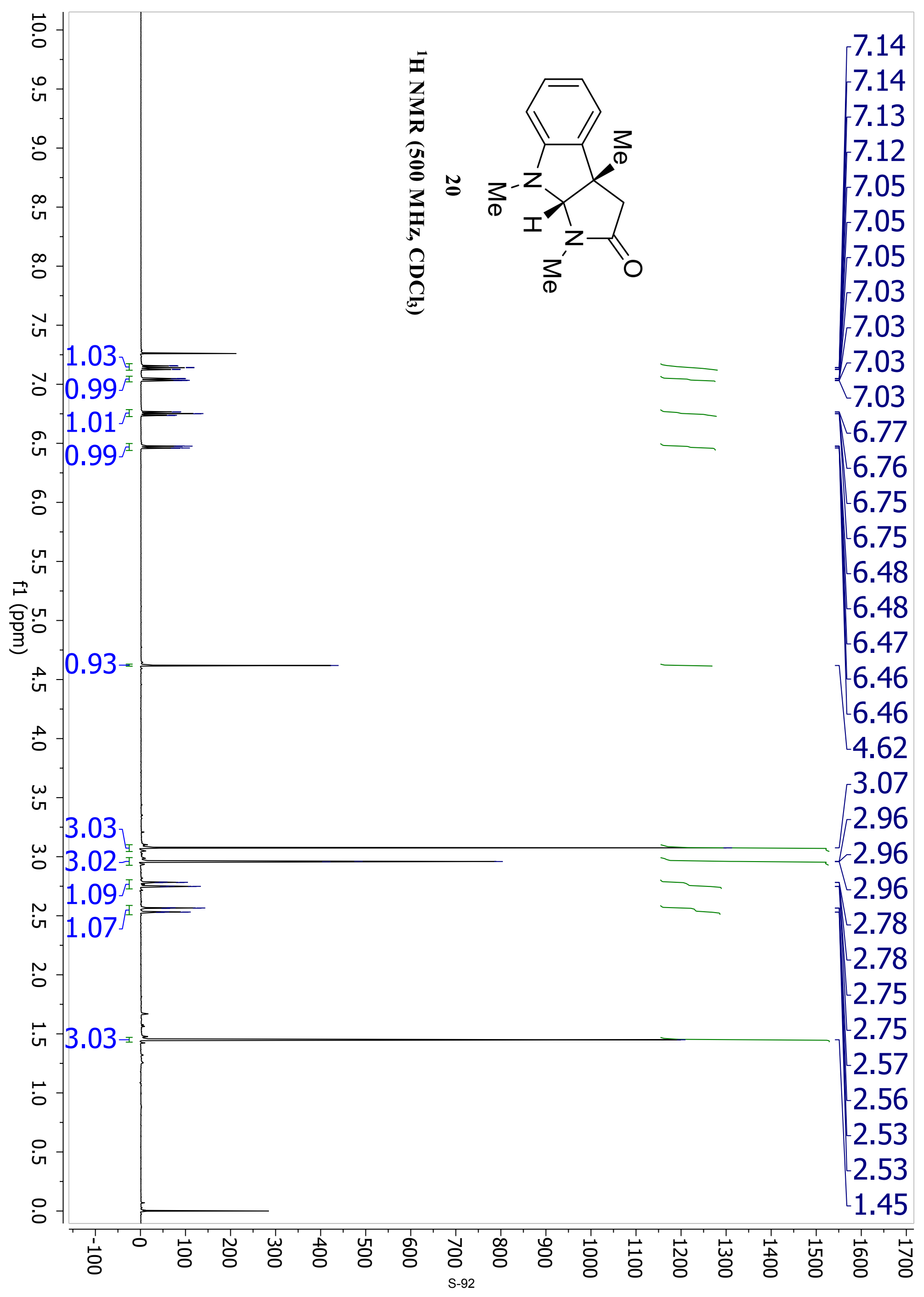




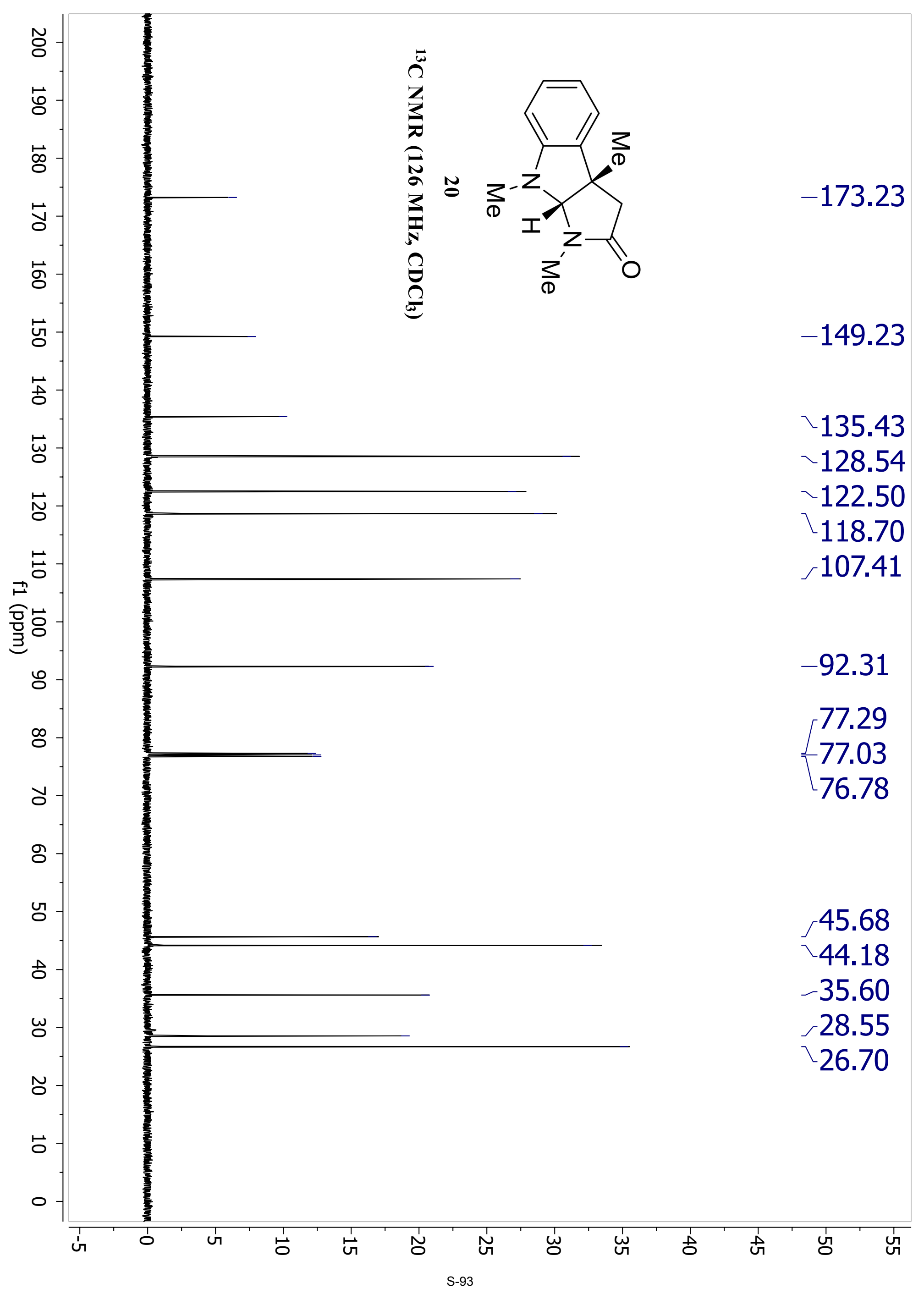




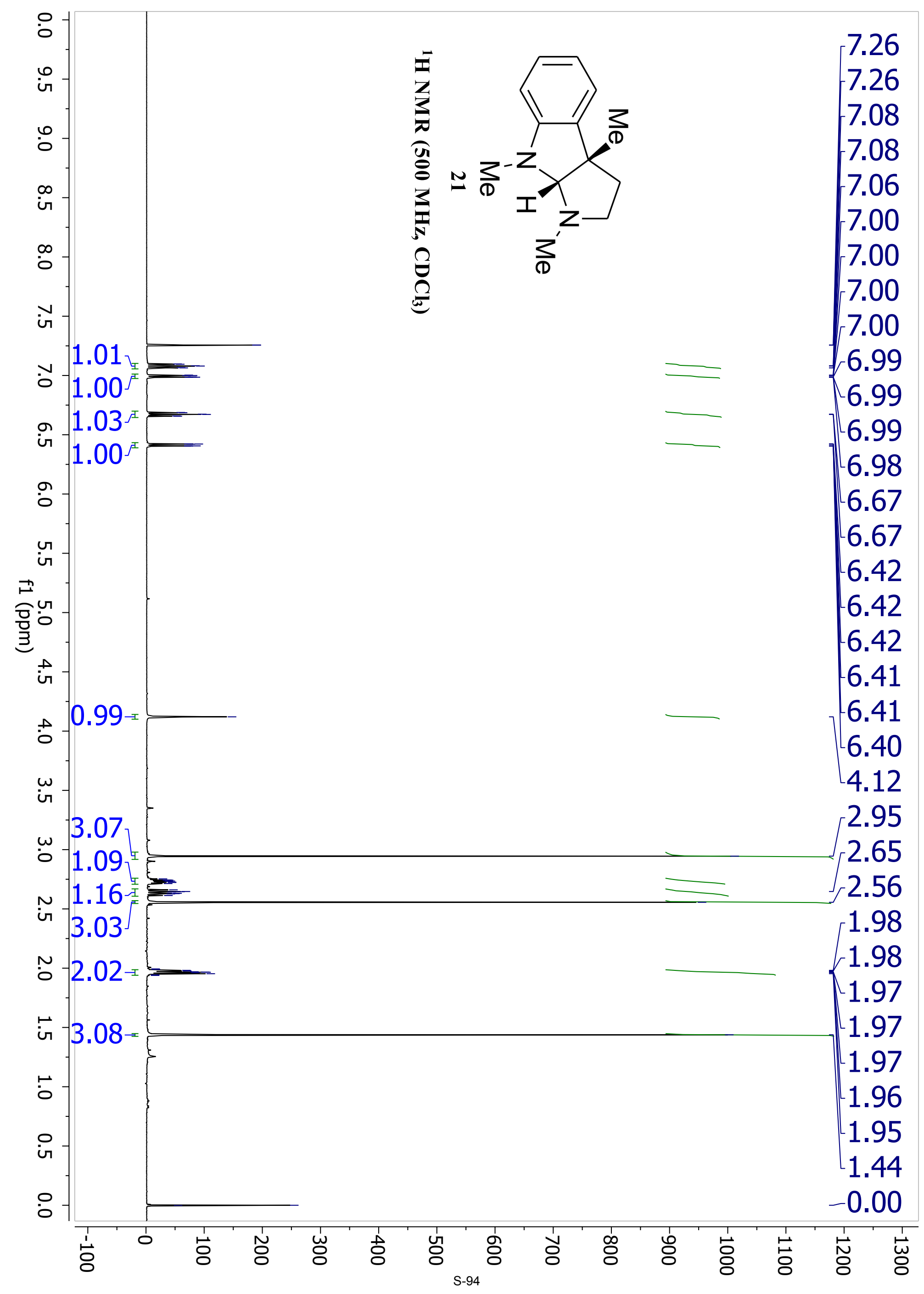




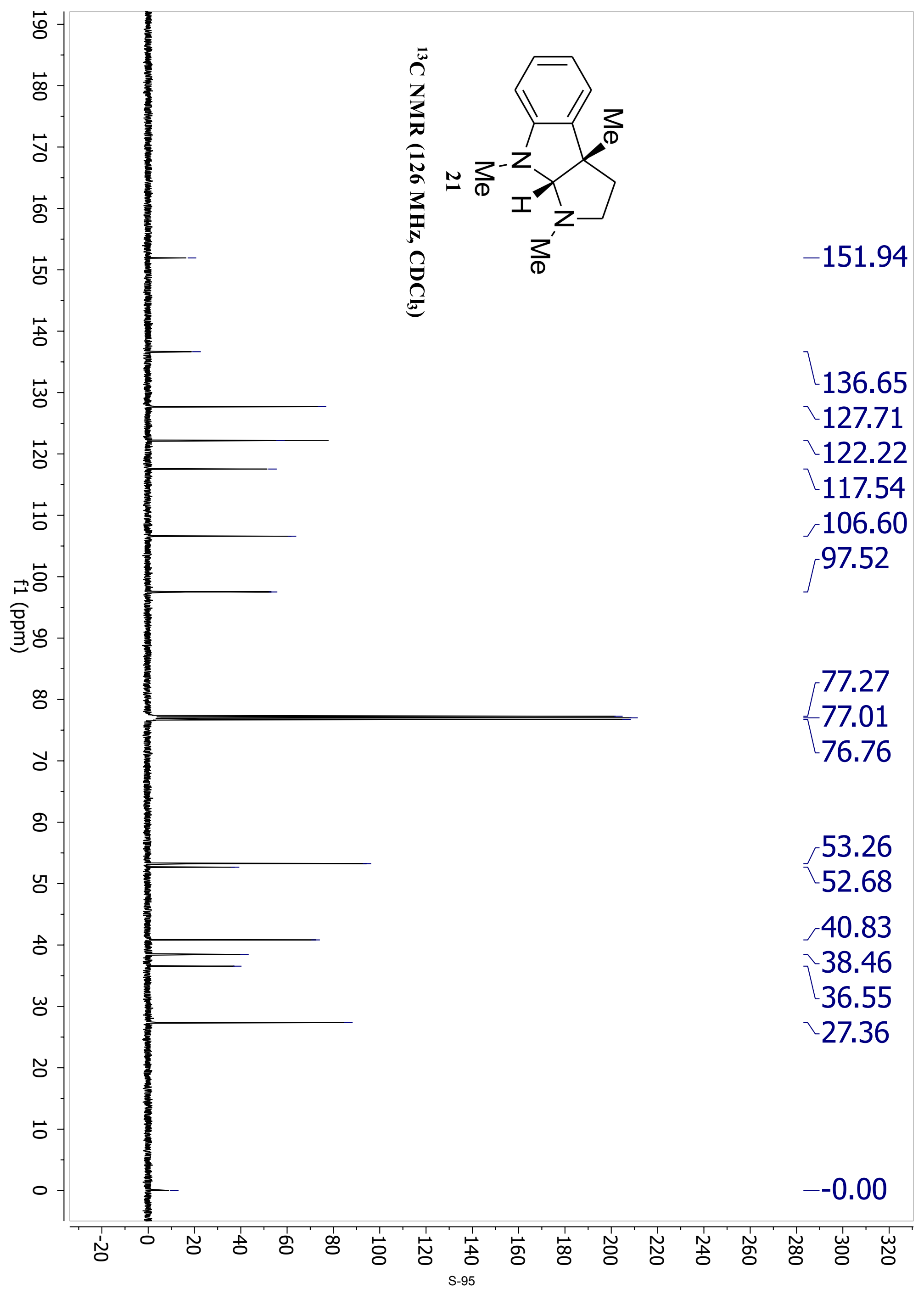




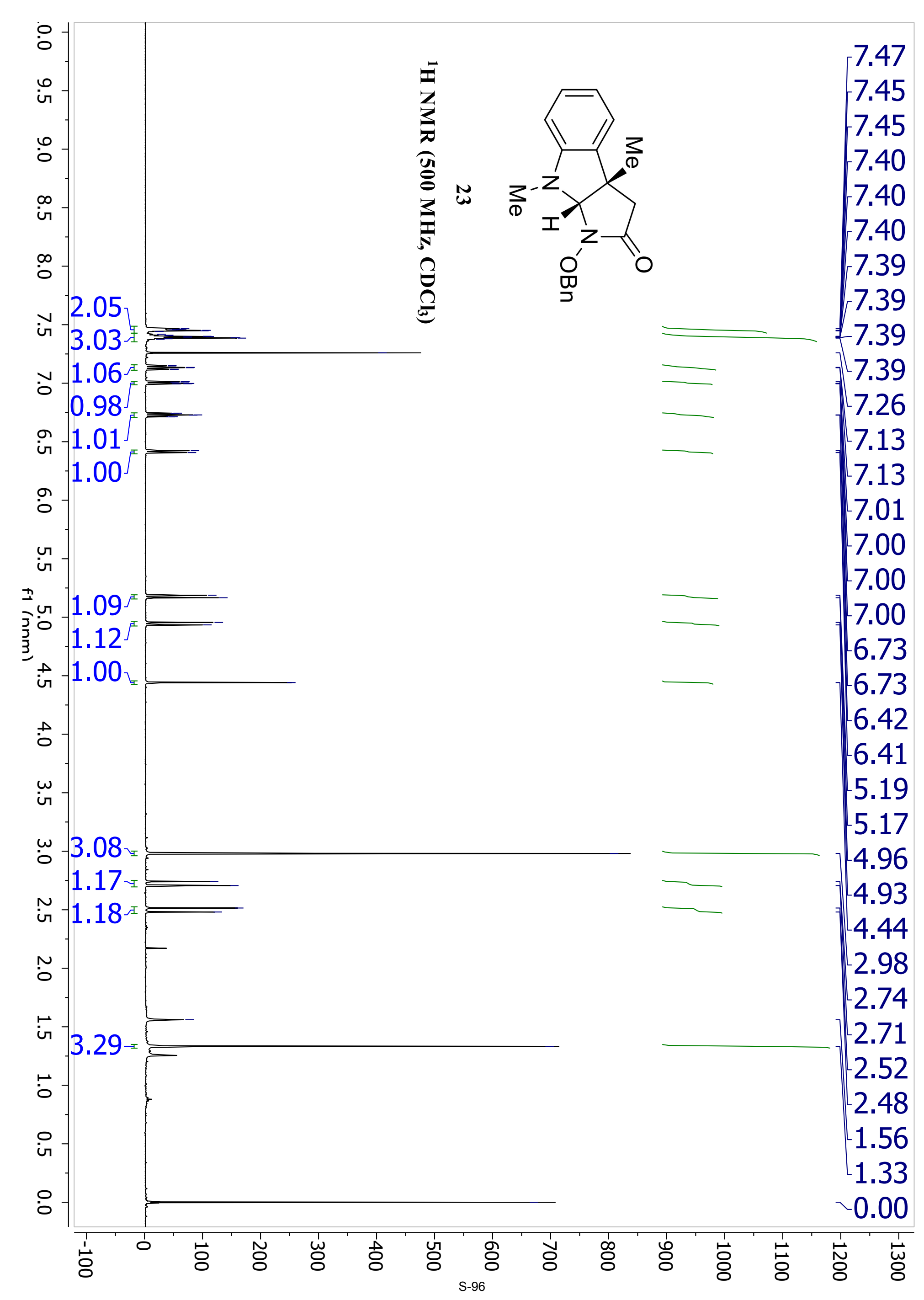




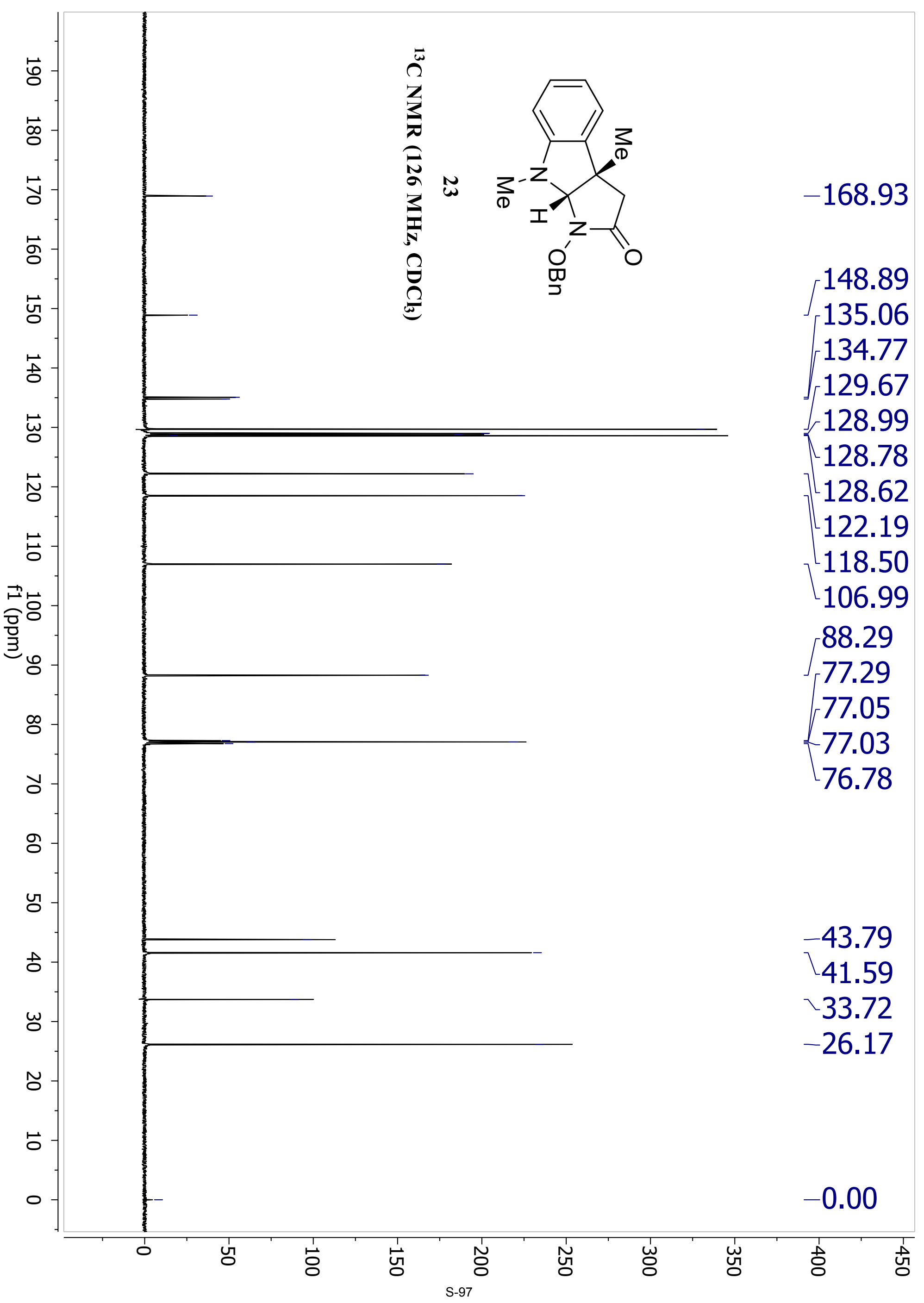




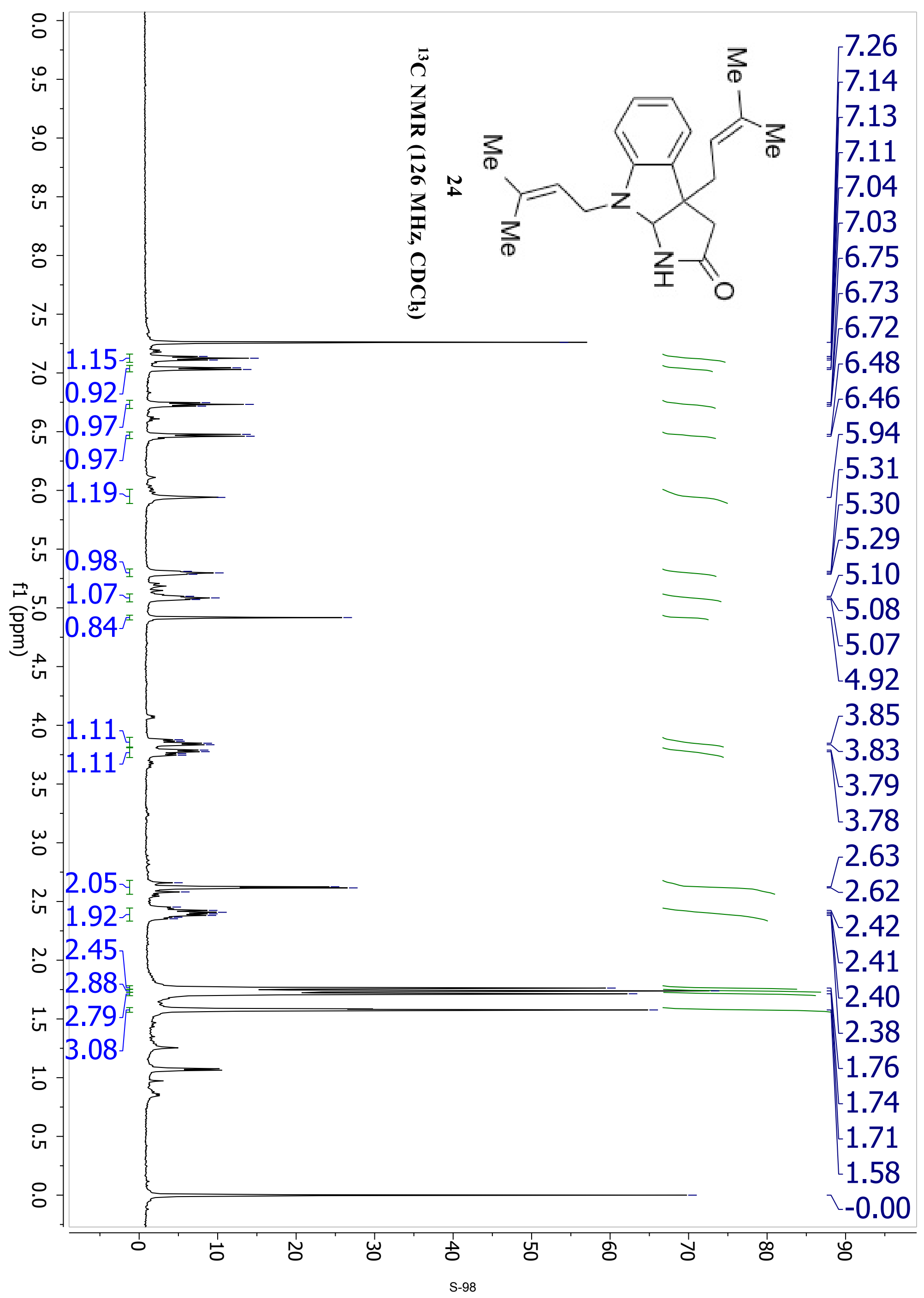




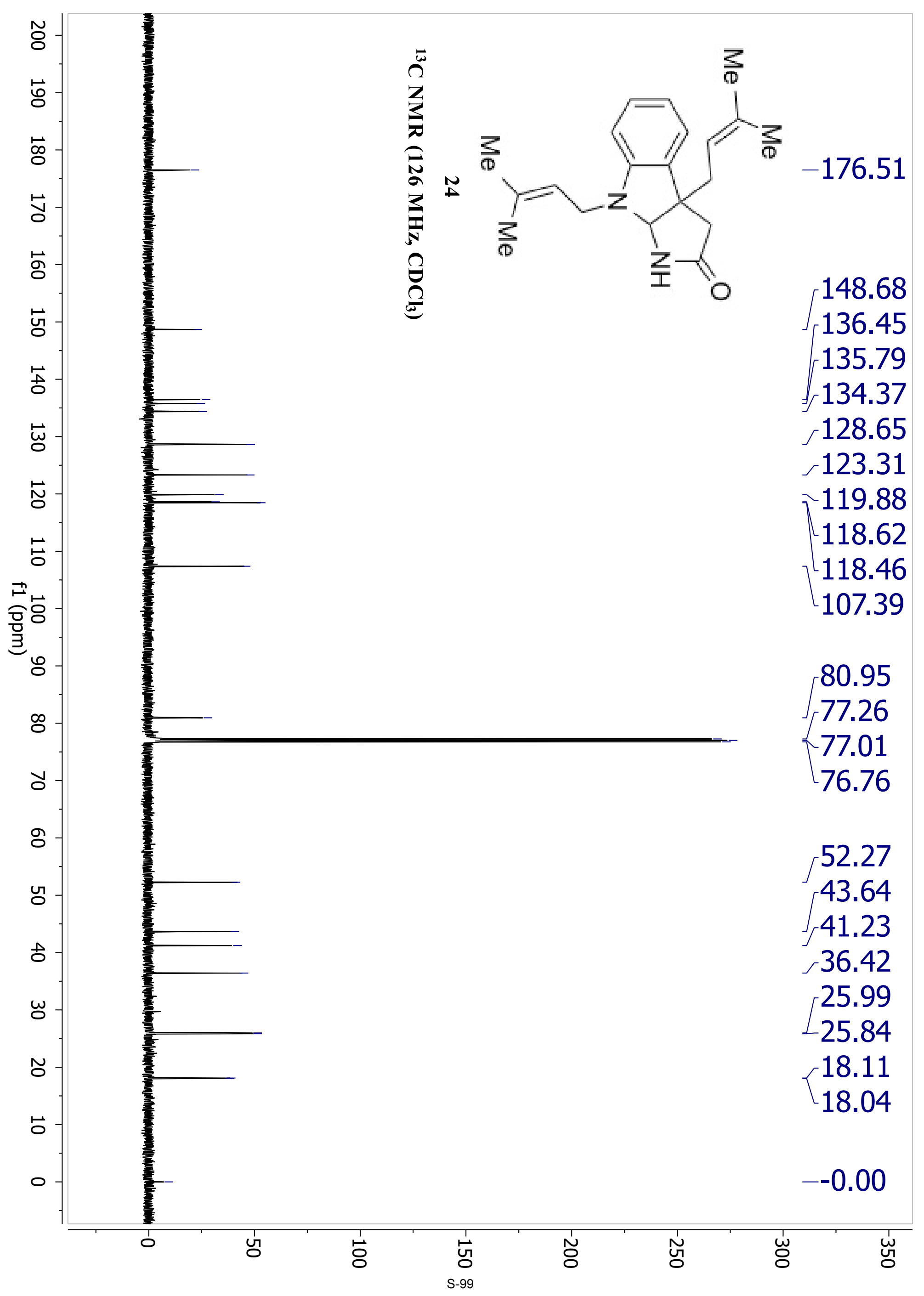

